\title{
Continuous Symmetries of Difference Equations
}

\author{
Decio Levi, \\ Dipartimento di Ingegneria Elettronica, \\ Università degli Studi Roma Tre and \\ I.N.F.N. Sezione di Roma III, \\ Via della Vasca Navale 84, 00146 Roma, Italy \\ email: levi@fis.uniroma3.it \\ Pavel Winternitz, \\ Centre de recherches mathématiques, \\ Université de Montréal, \\ C.P. 6128 , succ. Centre-ville, \\ Montréal QC H3C 3J7, Canada \\ email:wintern@CRM.UMontreal.CA
}

October 1, 2018

\begin{abstract}
Lie group theory was originally created more than 100 years ago as a tool for solving ordinary and partial differential equations. In this article we review the results of a much more recent program: the use of Lie groups to study difference equations. We show that the mismatch between continuous symmetries and discrete equations can be resolved in at least two manners. One is to use generalized symmetries acting on solutions of difference equations, but leaving the lattice invariant. The other is to restrict to point symmetries, but to allow them to also transform the lattice.
\end{abstract}

\section{Contents}

1 Introduction

2

2 Point Svmmetries of Difference Equations Defined on Fixed. Nontransforming Lattices

2.1 Lie point svmmetries of the discrete time Toda equation . . . . 8

2.2 Lie point svmmetries of $\mathrm{D} \Delta \mathrm{Es} \ldots \ldots \ldots \ldots$

2.3 Svmmetries of the Toda lattice . . . . . . . . . . . . . . . 12

2.4 Classification of differential equations on a lattice . . . . . . . . 15

2.5 Svmmetries of the Two Dimensional Toda equation . . . . . . . . 18 

3 Generalized Point Symmetries on a Fixed Uniform Lattice 19
3.1 Basic Concepts of Umbral Calculus . . . . . . . . . . . . 20
3.2 Umbral Calculus and Svmmetries of Linear Difference Equations 23
3.3 Svmmetries of the Time Dependent Schrödinger Equation on a Lattice 24
3.4 Svmmetries of the Discrete Heat Equation . . . . . . . . . . 25

4 Generalized Symmetries on Fixed Uniform Lattices 26

4.1 Generalized svmmetries of difference equations . . . . . . . . 26

4.2 The Toda svstem and its svmmetries . . . . . . . . . . . . . . . 29

4.2.1 The svmmetrv algebra for the Toda lattice . . . . . . . 34

4.2.2 Contraction of the svmmetrv algebras in the continuous limit 37

4.2.3 Bäcklund transformations for the Toda equation . . . . . 39

4.2.4 Relation between Bäcklund transformations and higher svmmetries 41

5 Point Svmmetries Transforming Solutions and Lattices 43

5.1 Lie Point Svmmetries of Ordinary Difference Schemes . . . . . . 43

5.1.1 Ordinarv Difference Schemes . . . . . . . . . . . . . . 43

5.1.2 Point Svmmetries of Ordinarv Difference Schemes . . . . 45

5.2 Examples . . . . . . . . . . . . . . . . . 47

5.3 Svmmetrv Preserving Discretization of Ordinarv Differential Equations 50

5.3 .1 General Comments . . . . . . . . . . . . . . . 50

$5.3 .2 \quad$ Svmmetries of Second Order ODEs . . . . . . . . . . . 51

5.3.3 Svmmetries of the Three-point Difference Schemes . . . . 52

5.3.4 Lagrangian formalism and solutions of three-point $\mathrm{O} \Delta \mathrm{S} . \quad 57$

6 Conclusions and Open Problems 61

\section{Introduction}

The symmetry theory of differential equations is well understood. it goes back to the classical work of Sophus Lie and is reviewed in numerous modern books and articles $[14,21,23,34,74,104,106-109,194,198,208,224-226,236]$. As a matter of fact, Lie group theory is the most general and useful tool we have for obtaining exact analytic solutions of large classes of differential equations, specially nonlinear ones.

The application of Lie group theory to discrete equations is much more recent and a vigorous development of the theory only started in the 1990-ties $[12,17,31,42,50-58,63,64,79,80,86,89-95,112,113,118-120,123-125,127-130$, 134-145,148-161,166-171,176,189, 199-201,207,209,213-216,218,233,237-239].

The purpose of this article is to provide a review of the progress made.

In this whole field of research one uses group theory to do for difference equations what has been done for differential ones. This includes generating new solutions from old ones, identifying equations that can be transformed into each other, performing symmetry reduction, and identifying integrable equations. 
When adapting the group theoretical approach from differential equations to difference ones, we must answer three basic questions:

1. What do we mean by symmetries?

2. How do we find the symmetries of a difference system?

3. What do we do with the symmetries, once we know them?

Let us first briefly review the situation for differential equations.

Let us consider a general system of differential equations

$$
E_{a}\left(x, u, u_{x}, u_{2 x}, \ldots u_{n x}\right)=0, \quad x \in \mathbb{R}^{p}, u \in \mathbb{R}^{q}, a=1, \ldots N,
$$

where $u_{n x}$ denotes all (partial) derivatives of $u$ of order $n$. The numbers $p, q, n$ and $N$ are all nonnegative integers.

We are interested in the symmetry group $\mathfrak{O}$ of system (1.1), i.e. in the local Lie group of local point transformations taking solutions of eq. (1.1) into solutions of the same equation. Point transformations in the space $X \times U$ of independent and dependent variables have the form

$$
\tilde{x}=\Lambda_{\lambda}(x, u), \quad \tilde{u}=\Omega_{\lambda}(x, u),
$$

where $\lambda$ denotes the group parameters. Thus

$$
\Lambda_{0}(x, u)=x, \quad \Omega_{0}(x, u)=u,
$$

and the inverse transformation $(\tilde{x}, \tilde{u}) \mapsto(x, u)$ exists, at least locally.

The transformations (1.2) of local coordinates in $X \times U$ also determine the transformations of functions $u=f(x)$ and of derivatives of functions. A group $\mathfrak{G}$ of local point transformations of $X \times U$ will be a symmetry group of system (1.1) if the fact that $u(x)$ is a solution implies that $\tilde{u}(\tilde{x})$ is also a solution.

How does one find the symmetry group $\mathfrak{G}$ ? Instead of looking for "global" transformations as in eq. (1.2) one looks for infinitesimal ones, i.e. one looks for the Lie algebra $\mathfrak{g}$ that corresponds to $\mathfrak{G}$. A one-parameter group of infinitesimal point transformations will have the form

$$
\begin{aligned}
& \tilde{x}_{i}=x_{i}+\lambda \xi_{i}(x, u), \quad \tilde{u}_{\alpha}=u_{\alpha}+\lambda \phi_{\alpha}(x, u), \\
& |\lambda| \ll 1 \quad 1 \leq i \leq p, \quad 1 \leq \alpha \leq q .
\end{aligned}
$$

The search for the symmetry algebra of a system of differential equations is best formulated in terms of vector fields acting on the space $X \times U$ of independent and dependent variables. Indeed, consider the vector field

$$
\hat{X}=\sum_{i=1}^{p} \xi_{i}(x, u) \partial_{x_{i}}+\sum_{\alpha=1}^{q} \phi_{\alpha}(x, u) \partial_{u_{\alpha}},
$$

where the coefficients $\xi_{i}$ and $\phi_{\alpha}$ are the same as in eq. (1.3). If these functions are known, the vector field (1.4) can be integrated to obtain the finite transformations (1.2). Indeed, all we have to do is to integrate the equations

$$
\frac{d \tilde{x}_{i}}{d \lambda}=\xi_{i}(\tilde{x}, \tilde{u}), \quad \frac{d \tilde{u}_{\alpha}}{d \lambda}=\phi_{\alpha}(\tilde{x}, \tilde{u})
$$


subject to the initial conditions

$$
\left.\tilde{x}_{i}\right|_{\lambda=0}=\left.x_{i} \quad \tilde{u}_{\alpha}\right|_{\lambda=0}=u_{\alpha} .
$$

This provides us with a one-parameter group of local Lie point transformations of the form (1.2) where $\lambda$ is the group parameter.

The vector field (1.4) tells us how the variables $x$ and $u$ transform. We also need to know how derivatives like $u_{x}, u_{x x}, \ldots$ transform. This is given by the prolongation of the vector field $\hat{X}$.

We have

$$
\begin{aligned}
\operatorname{pr} \hat{X}=\hat{X}+\sum_{\alpha}\left\{\sum_{i} \phi_{\alpha}^{x_{i}} \partial_{u_{\alpha, x_{i}}}+\sum_{i, k} \phi_{\alpha}^{x_{i} x_{k}} \partial_{u_{\alpha, x_{i} x_{k}}}\right. & \\
& \left.+\sum_{i, k, l}^{x_{i} x_{k} x_{l}} \phi_{\alpha}^{x_{i} x_{k} x_{l}} \partial_{u_{\alpha, x_{i} x_{k} x_{l}}}+\ldots\right\},
\end{aligned}
$$

where the coefficients in the prolongation can be calculated recursively, using the total derivative operator,

$$
D_{x_{i}}=\partial_{x_{i}}+u_{\alpha, x_{i}} \partial_{u_{\alpha}}+u_{\alpha, x_{a} x_{i}} \partial_{u_{\alpha, x_{a}}}+u_{\alpha, x_{a} x_{b} x_{i}} \partial_{u_{\alpha, x_{a} x_{b}}}+\ldots
$$

(a summation over repeated indices is to be understood). The recursive formulas are

$$
\begin{aligned}
& \phi_{\alpha}^{x_{i}}=D_{x_{i}} \phi_{\alpha}-\left(D_{x_{i}} \xi_{a}\right) u_{\alpha, x_{a}}, \quad \phi_{\alpha}^{x_{i} x_{k}}=D_{x_{k}} \phi_{\alpha}^{x_{i}}-\left(D_{x_{k}} \xi_{a}\right) u_{\alpha, x_{i} x_{a}},(1.9) \\
& \phi_{\alpha}^{x_{i} x_{k} x_{l}}=D_{x_{l}} \phi_{\alpha}^{x_{i} x_{k}}-\left(D_{x_{l}} \xi_{a}\right) u_{\alpha, x_{i} x_{k} x_{a}}
\end{aligned}
$$

etc.

The invariance condition for system (1.1) is expressed in terms of the operator (1.7) as

$$
\left.\operatorname{pr}^{(n)} \hat{X} E_{a}\right|_{E_{1}=\cdots=E_{N}=0}=0, \quad a=1, \ldots N,
$$

where $\operatorname{pr}^{(n)} \hat{X}$ is the prolongation (1.7) calculated up to order $n$ (where $n$ is the order of system (1.1).

Eq. (1.10) provides a system of linear partial differential equations for the functions $\xi_{i}(x, u)$ and $\phi_{\alpha}(x, u)$, in which the variables $x$ and $u$ figure as independent variables. By definition of point transformations the coefficients $\xi_{i}$ and $\phi_{\alpha}$ depend only on $\left(x_{1}, \ldots, x_{p}, u_{1}, \ldots, u_{q}\right)$, not on any derivative of $u_{\alpha}$. The action of $p r^{(n)} \hat{X}$ in eq. (1.10) will, on the other hand, introduce terms in (1.10), involving the derivatives $\frac{\partial^{k} u}{\partial x_{1}^{k_{1}} \ldots \partial x_{p}^{k_{p}}}, k=k_{1}+\ldots+k_{p}, 1 \leq k \leq n$. We use equations (1.1) to eliminate $N$ (the number of equations) such derivatives. We then collect all linearly independent remaining expressions in the derivatives and set the coefficients of these expressions equal to zero. This provides the "determining equations": a set of linear partial differential equations for the functions $\xi_{i}(x, u)$ and $\phi_{\alpha}(x, u)$. The order of the system of determining equations is the same as the order of the studied system (1.1), however the determining system 
is linear, even if the system (1.1) is nonlinear. It is usually overdetermined and not difficult to solve. Computer programs using various symbolic languages exist that derive the determining system and solve it, or at least partially solve it $[35,88,221,224]$.

The solution of the determining system may be trivial, i.e. $\xi_{i}=0, \phi_{\alpha}=0$. Then the symmetry approach is of no avail. Alternatively, the general solution may depend on a finite number $K$ of integration constants. The Lie algebra of the symmetry group, the "symmetry algebra", for short, is then $K$-dimensional and must be identified as an abstract Lie algebra $[87,111,181,205]$. Finally the general solution of the determining equations may involve arbitrary functions and the symmetry algebra is infinite-dimensional.

So far we have considered only point transformations, as in eq. (1.2), in which the new variables $\tilde{x}$ and $\tilde{u}$ depend only on the old ones, $x$ and $u$. More general transformations are "contact transformations", where $\tilde{x}$ and $\tilde{u}$ also depend on first derivatives of $u[15,23,104,106,194,226]$. A still more general class of transformations are generalized transformations, also called "Lie-Bäcklund" transformations $[15,114,194]$. For these, the transformations are

$$
\tilde{x}=\Lambda_{\lambda}\left(x, u, u_{x}, u_{x x}, \ldots\right), \quad \tilde{u}=\Omega_{\lambda}\left(x, u, u_{x}, u_{x x}, \ldots\right),
$$

involving derivatives up to an arbitrary, but finite order. The coefficients $\xi_{i}$ and $\phi_{\alpha}$ of the vector fields (1.4) will then also depend on derivatives of $u$.

When studying generalized symmetries, and sometimes also point symmetries, it is convenient to use a different formalism, namely that of evolutionary vector fields. Let us first consider the case of Lie point symmetries, i.e. vector fields of the form (1.4) and their prolongations (1.7). To each vector field (1.4) we can associate its evolutionary counterpart $\hat{X}_{e}$, defined as

$$
\hat{X}_{e}=Q_{\alpha}\left(x, u, u_{x}\right) \partial_{u_{\alpha}}, \quad Q_{\alpha}=\phi_{\alpha}-\xi_{j} u_{\alpha, x_{j}} .
$$

The prolongation of the evolutionary vector field (1.12) is defined as

$$
\begin{aligned}
\operatorname{pr} \hat{X}_{e} & =Q_{\alpha} \partial_{u_{a}}+Q_{\alpha}^{x_{j}} \partial_{u_{\alpha, x_{j}}}+Q_{\alpha}^{x_{j} x_{k}} \partial_{u_{\alpha, x_{j} x_{k}}}+\ldots \\
Q_{\alpha}^{x_{j}} & =D_{x_{j}} Q_{\alpha}, \quad Q_{\alpha}^{x_{j} x_{k}}=D_{x_{j}} D_{x_{k}} Q_{\alpha}, \ldots
\end{aligned}
$$

The functions $Q_{\alpha}$ are called the characteristics of the vector field. Observe that $\hat{X}_{e}$ and $\operatorname{pr} \hat{X}_{e}$ do not act on the independent variables $x_{j}$. For Lie point symmetries evolutionary and ordinary vector fields are entirely equivalent and it is easy to pass from one to the other. Indeed, eq. (1.12) gives the connection between the two. The symmetry algorithms for calculating the symmetry algebra $\mathfrak{g}$ in terms of ordinary, or evolutionary vector fields, are also equivalent. Eq. 1.10 is simply replaced by

$$
\left.\operatorname{pr}^{(n)} \hat{X}_{e} E_{a}\right|_{E_{1}=\cdots=E_{N}=0},=0, \quad a=1, \ldots N .
$$

The reason that eq. (1.10) and (1.14) are equivalent is the following:

$$
\operatorname{pr}^{(n)} \hat{X}_{e}=\operatorname{pr}^{(n)} X-\xi_{i} D_{i}
$$


The total derivative $D_{i}$ is itself a generalized symmetry of eq. (1.1), i.e.,

$$
\left.D_{i} E_{a}\right|_{E_{1}=E_{2}=\cdots=E_{N}=0},=0 \quad i=1, \ldots p, \quad a=1, \ldots N .
$$

Eqs. (1.15) and (1.16) prove that systems (1.10) and (1.14) are equivalent. Eq. (1.16) itself follows from the fact that $D_{i} E_{a}=0$ is a differential consequence of eq. (1.1), hence every solution of eq. (1.1) is also a solution of eq. (1.16).

To find generalized symmetries of order $k$, we use eq. (1.12) but allow the characteristics $Q_{\alpha}$ to depend on all derivatives of $u$ up to order $k$. The prolongation is calculated using eq. (1.13). The symmetry algorithm is again eq. (1.14).

A very useful property of evolutionary symmetries is that $Q_{\alpha}$ provide compatible flows. This means that the system of equations

$$
\frac{\partial u_{\alpha}}{\partial \lambda}=Q_{\alpha}
$$

is compatible with system (1.1). In particular, group invariant solutions, i.e., solutions invariant under a subgroup of $\mathfrak{G}$, are obtained as fixed points

$$
Q_{\alpha}=0 .
$$

If $Q_{\alpha}$ is the characteristic of a point transformation, then (1.18) is a system of quasilinear first order partial differential equations. They can be solved and their solutions can be substituted into (1.1), yielding the invariant solutions explicitly.

Many different extensions of Lie's original method of group invariant solutions exist. Among them we mention, first of all, conditional symmetries $[22,67,68,147]$. For differential equations, they were introduced under several different names $[22,41,147,195]$ in order to obtain dimensional reductions of partial differential equations, beyond those obtained by using ordinary Lie symmetries.

Another valuable extension is the concept of partial symmetries. They correspond to the existence of a subset of solutions which, without necessarily being invariant, are mapped into each other by the transformation [39,40]. Further extensions are given by asymptotic symmetries [75,77], when extra symmetries are obtained in the asymptotic regime, or approximate symmetries $[16,69,109]$ where one considers the symmetries of approximate solutions of a system depending on a small parameter.

Let us now return to the problem at hand, namely symmetries of difference systems. We wish to study the continuous symmetries and use Lie algebra techniques. However, the equations are now discrete, i.e. they involve functions $u(x)$ that are themselves continuous, but evaluated, or sampled, at discrete points. Several different approaches to this problem have been developed and are discussed below.

In Section 2 we discuss point symmetries of difference equations defined on fixed, nontransforming lattices [31,79,86,118-120,123,148,151,156,167-170,176, $200,201,214,216]$. The symmetry transformations are assumed to have the form 
(1.2). They must take solutions into solutions and the lattice into itself. This approach is fruitful mainly for differential-difference equations (D $\Delta \mathrm{E}$ 's), where not only the dependent variables, but also some of the independent ones are continuous.

Section 3 is devoted to generalized point symmetries on fixed lattices [63, $64,93,128,130,139,140,145]$. The concept of symmetry is generalized in that transformations act simultaneously at several points of the lattice, possibly infinitely many ones. In the continuous limit they reduce to point transformations. This approach is fruitful mainly for linear equations, or equations that can be linearized by a transformation of variables.

In Section 4 we consider generalized symmetries on fixed lattices [89-91,95, 127] This approach is fruitful for discrete nonlinear integrable equations, i.e., nonlinear difference equations possessing a Lax pair. The symmetries are generalized ones, treated in the evolutionary formalism. In a continuous limit they reduce to point and generalized symmetries of integrable differential equations.

Point symmetries transforming solutions and lattices $[17,50-52,54-58,141$, 143, 157] are considered in Section [5] The transformations have the form (1.2) and they act on solutions and on lattices simultaneously. The lattices themselves are given by difference equations and their form is dictated by the symmetries. Their main application is to discretize given differential equations while preserving their symmetries.

A brief conclusion with an overview of the results presented and pending issues is given in Section 6

\section{Point Symmetries of Difference Equations De- fined on Fixed, Nontransforming Lattices}

The essentially continuous techniques for finding Lie symmetries for differential equations can be extended in a natural way to the discrete case by acting just on the continuous variables $[148,151,167-170,200]$, leaving the lattice invariant. Transformations of the lattice are considered only at the level of the group which itself is finite or discrete. Depending on the discrete equation we are considering we can have translations on the lattice by multiples of the lattice spacing and rotations through fixed angles, i.e., for example, by $\frac{\pi}{2}$ when the partial difference equation is invariant with respect to the interchange of the two lattice variables.

In Section 2.1 we consider, as an example, the Lie point symmetries of the discrete time Toda lattice [127]. In Section 2.2 we review the steps necessary to obtain continuous symmetries for differential-difference equations (D $\Delta \mathrm{E}$ 's) and then in Section 2.3 we carry out the explicit calculations in the case of the Toda equation in $1+1$ dimensions. In doing so we will present and compare the results contained in our articles on the subject, which correspond to both the intrinsic method and the differential equation method proposed earlier, and those of the global method introduced by Quispel et al. [200].

Apart from the standard Toda lattice we will consider a Toda lattice equation 
with variable coefficients and will show how, by analyzing its symmetry group, one can find the Lie point transformation which maps it into the standard Toda lattice equation with constant coefficients. In Section 2.4 we will present some results on the classification of nonlinear $\mathrm{D} \Delta \mathrm{E}$ 's with nearest neighbour interac-

tions. Finally, symmetries of a two-dimensional Toda lattice are discussed in Section 2.5

\subsection{Lie point symmetries of the discrete time Toda equa- tion}

The discrete time Toda equation [100] is one of the integrable completely discrete partial differential equations $(\Delta \Delta \mathrm{E})[11,99,101-103,131,186,187,190,196,217$, $219,220,227]$ and is given by

$$
\begin{aligned}
\Delta_{\text {Toda }} & =e^{u_{n, m}-u_{n, m+1}}-e^{u_{n, m+1}-u_{n, m+2}}- \\
& -\alpha^{2}\left(e^{u_{n-1, m+2}-u_{n, m+1}}-e^{u_{n, m+1}-u_{n+1, m}}\right)=0 .
\end{aligned}
$$

On the left hand side of eq. (2.1) we can easily obtain the second difference of the function $u_{n, m}$ with respect to the discrete-time $m$. Thus, defining

$$
t=m \sigma_{t} ; \quad v_{n}(t)=u_{n, m} ; \quad \alpha=\sigma_{t}^{2}
$$

we find that eq.(2.1) reduces to the continuous-time Toda equation:

$$
\Delta_{\text {Toda }}^{(2)}=v_{n, t t}-e^{v_{n-1}-v_{n}}+e^{v_{n}-v_{n+1}}=0,
$$

when $\sigma_{t} \rightarrow 0$ and $m \rightarrow \infty$ in such a way that $t$ remains finite. The Toda equation (2.3) is probably the best known and most studied differential-difference equation. It plays, in the case of lattice equations, the same role as the Korteweg - de Vries equation for partial differential equations [230,231]. It was obtained by Toda [229] when trying to explain the Fermi, Pasta and Ulam results [62] on the numerical experiments on the equipartition of energy in a nonlinear lattice of interacting oscillators. As shown below in Section 4.2.2 eq. (2.3) reduces, in the continuum limit, to the potential Korteweg-de Vries equation. It can be encountered in many applications from solid state physics to DNA biology, from molecular chain dynamics to chemistry [206].

Eq.(2.1) can be obtained as the compatibility condition of the following overdetermined pair of linear difference equations:

$$
\begin{array}{r}
\psi_{n-1, m}+\left(\alpha+\frac{1}{\alpha}-\alpha e^{u_{n-1, m+1}-u_{n, m}}-\frac{e^{u_{n, m}-u_{n, n+1}}}{\alpha}\right) \psi_{n, m} \\
+e^{u_{n, m}-u_{n+1, m}} \psi_{n+1, m}=\lambda \psi_{n, m} \\
\psi_{n, m+1}=\psi_{n, m}-\alpha e^{u_{n, m+1}-u_{n+1, m}} \psi_{n+1, m}
\end{array}
$$

Let us consider the Lie point symmetries of the discrete-time Toda lattice (2.1), on a fixed nontrasforming bidimensional lattice characterized by two lattice spacings in the two directions $m$ and $n, \sigma_{t}$ and $\sigma_{x}$. 
If the lattice is uniform and homogeneous in both variables, we can represent the lattice by the following set of equations:

$$
x_{n, m}=n \sigma_{x}, \quad t_{n, m}=m \sigma_{t} .
$$

Eqs. (2.6) from now on will be denoted as $\Delta_{\text {Lattice }}=0$.

A Lie point symmetry is defined by giving its infinitesimal generators, i.e. the vector field

$$
\begin{aligned}
\hat{X}_{n, m} & =\xi_{n, m}\left(x_{n, m}, t_{n, m}, u_{n, m}\right) \partial_{x_{n, m}}+ \\
& +\tau_{n, m}\left(x_{n, m}, t_{n, m}, u_{n, m}\right) \partial_{t_{n, m}}+\phi_{n, m}\left(x_{n, m}, t_{n, m}, u_{n, m}\right) \partial_{u_{n, m}},
\end{aligned}
$$

and generates an infinitesimal transformation in the site $(n, m)$ of its coordinates and of the function $u\left(x_{n, m}, t_{n, m}\right)=u_{n, m}$. The action of (2.7) on the discretetime Toda lattice equation (2.1) is obtained by prolonging (2.7) to all points of the lattice. The prolongation is obtained $[143,148,151]$ by shifting (2.7) to these points

$$
\operatorname{pr} \hat{X}=\hat{X}_{n, m}+\hat{X}_{n+1, m}+\hat{X}_{n, m+1}+\hat{X}_{n, m+2}+\hat{X}_{n-1, m+2} .
$$

The invariance condition then reads:

$$
\left.\operatorname{pr} \hat{X} \Delta_{\text {Toda }}\right|_{\left(\Delta_{\text {Toda }}=0, \Delta_{\text {Lattice }}=0\right)}=0,\left.\quad \operatorname{pr} \hat{X} \Delta_{\text {Lattice }}\right|_{\left(\Delta_{\text {Toda }}=0, \Delta_{\text {Lattice }}=0\right)}=0(2.9
$$

The action of (2.8) on the lattice equation (2.6) gives $\xi_{n, m}=0, \tau_{n, m}=0$. When we act with 2.8 on the Toda equation, we get

$$
\begin{array}{r}
e^{u_{n, m}-u_{n, m+1}\left[\phi_{n, m}-\phi_{n, m+1}\right]-e^{u_{n, m+1}-u_{n, m+2}}\left[\phi_{n, m+1}-\phi_{n, m+2}\right]-} \\
-\alpha^{2}\left\{e^{u_{n-1, m+2}-u_{n, m+1}}\left[\phi_{n-1, m+2}-\phi_{n, m+1}\right]-\right. \\
\left.-e^{u_{n, m+1}-u_{n+1, m}}\left[\phi_{n, m+1}-\phi_{n+1, m}\right]\right\}=0
\end{array}
$$

From the action of $\hat{X}$ on (2.6) the infinitesimal coefficients $\xi$ and $\tau$ are zero and thus the variables $x$ and $t$ are invariant. If we differentiate eq. (2.10) twice with respect to $u_{n, m+2}$ we get that $\phi_{n, m}=c_{1} e^{u_{n, m}}+c_{2}$, where $c_{1}$ and $c_{2}$ are two integration constants (that can depend on $x_{n, m}$ and $t_{n, m}$ ). Introducing this result into eq. 2.10) we get that $c_{1}$ must be equal to zero. Taking into account that, due to the form of the lattice, all points are independent we get that $c_{2}$ must be just a constant.

To sum up, the discrete time Toda equation (2.1) considered on a fixed lattice has only a one-dimensional continuous symmetry group. It consists of the translation of the dependent variable $u$, i.e. $\tilde{u}_{n, m}=u_{n, m}+\lambda$ with $\lambda$ constant. This symmetry is obvious from the beginning as eq. (2.1) does not involve $u_{n, m}$ itself but only differences between values of $u$ at different points of the lattice. Other transformations that leave the lattice and solutions invariant will be discrete [138]. In this case they are simply translations of $x$ and $t$ by integer multiples of the lattice spacing $\sigma_{x}$ and $\sigma_{t}$. 
The same conclusion holds in the general case of difference equations on fixed lattices. Lie algebra techniques will provide transformations of the continuous dependent variables only, through the transformations can depend on the discrete independent variables.

In Section 4 we will see that the situation is completely different when generalized symmetries are considered. In Section 5 we will consider transforming lattices, which greatly increases the role of point symmetries.

\section{$2.2 \quad$ Lie point symmetries of $\mathrm{D} \Delta \mathrm{Es}$}

Let us now consider the more interesting case of D $\Delta$ Es. For notational simplicity, let us restrict ourselves to scalar D $\Delta$ Es for one real function $u(n, t)$ depending on one lattice variable $n$ and one continuous real variable, $t$. Moreover, we will only be interested in $\mathrm{D} \Delta$ Es containing up to second order derivatives, as those are the ones of particular interest in applications to dynamical systems. We write such equations as:

$$
\begin{aligned}
& \Delta_{n}^{(2)} \equiv \Delta\left(t, n,\left.u_{n+k}\right|_{k=a_{0}} ^{b_{0}},\left.u_{n+k, t}\right|_{k=a_{1}} ^{b_{1}},\left.u_{n+k, t t}\right|_{k=a_{2}} ^{b_{2}}\right)=0 \\
& a_{j} \leq b_{j} \in \mathbb{Z},
\end{aligned}
$$

with $u_{n} \equiv u_{n}(t)$.

The lattice is uniform, time independent and fixed, the continuous variable $t$ is the same at all points of the lattice. Thus to eq. (2.11) we add

$$
t_{n}=t_{n+1}=t
$$

Examples of such equations which will be considered in the following are the Toda lattice equation (2.3) and the inhomogeneous Toda lattice [133]

$$
\begin{aligned}
& \tilde{\Delta}_{n}^{(2)}=w_{, t t}(n)-\frac{1}{2} w_{, t}+\frac{1}{4}-\frac{n}{2}+\left[\frac{1}{4}(n-1)^{2}+1\right] e^{w(n-1)-w(n)} \\
&-\left[\frac{1}{4} n^{2}+1\right] e^{w(n)-w(n+1)}=0 .
\end{aligned}
$$

We are interested in Lie point transformations which leave the solution set of eqs. (2.11), (2.12) invariant. They have the form:

$$
\tilde{t}=\Lambda_{g}\left(t, n, u_{n}(t)\right), \quad \tilde{u}_{\tilde{n}}(\tilde{t})=\Omega_{g}\left(t, n, u_{n}(t)\right), \quad \tilde{n}=n
$$

where $g$ represents a set of continuous or discrete group parameters.

Continuous transformations of the form (2.14) are generated by a Lie algebra of vector fields of the form:

$$
\widehat{X}=\tau_{n}\left(t, u_{n}(t)\right) \partial_{t}+\phi_{n}\left(t, u_{n}(t)\right) \partial_{u_{n}}
$$

where $n$ is treated as a discrete variable and we have set $\tilde{n}=n$, when considering continuous transformations.

Invariance of the condition (2.12) implies that $\tau$ does not depend on $n$. 
As in the case of purely differential equations, the following invariance condition

$$
\left.\operatorname{pr}^{(2)} \widehat{X} \Delta_{n}^{(2)}\right|_{\Delta_{n}^{(2)}=0}=0
$$

must be true if $\widehat{X}$ is to belong to the Lie symmetry algebra of $\Delta_{n}^{(2)}$. The symbol $\operatorname{pr}^{(2)} \widehat{X}$ denotes the second prolongation of the vector field $\widehat{X}$, i.e.

$$
\begin{aligned}
\operatorname{pr}^{(2)} \widehat{X}=\tau\left(t, u_{n}\right) \partial_{t} & +\sum_{k=n-a}^{n+b} \phi_{k}\left(t, u_{k}\right) \partial_{u_{k}} \\
& +\sum_{k=n-a_{1}}^{n+b_{1}} \phi_{k}^{t}\left(t, u_{k}, u_{k, t}\right) \partial_{u_{k, t}} \\
& +\sum_{k=n-a_{2}}^{n+b_{2}} \phi_{k}^{t t}\left(t, u_{k}, u_{k, t}, u_{k, t t}\right) \partial_{u_{k, t t}}
\end{aligned}
$$

with

$$
\begin{aligned}
\phi_{k}^{t}\left(t, u_{k}, u_{k, t}\right) & =D_{t} \phi_{k}\left(t, u_{k}\right)-\left[D_{t} \tau_{k}\left(t, u_{k}\right)\right] u_{k, t} \\
\phi_{k}^{t t}\left(t, u_{k}, u_{k, t}, u_{k, t t}\right) & =D_{t} \phi_{k}^{t}\left(t, u_{k}, u_{k, t}\right)-\left[D_{t} \tau_{k}\left(t, u_{k}\right)\right] u_{k, t t}
\end{aligned}
$$

where $D_{t}$ is the total derivative with respect to $t$.

Let us notice moreover that $\phi^{t}$ and $\phi^{t t}$ are the prolongation coefficients with respect to the continuous variable. The prolongation with respect to the discrete variable is reflected in the summation over $k$.

Eq. (2.16) is to be viewed as just one equation with $n$ as a discrete variable; thus we have a finite algorithm for obtaining the determining equations, a usually overdetermined system of linear partial differential equations for $\tau\left(t, u_{n}\right)$ and $\phi\left(n, t, u_{n}\right)$.

A different approach consists of considering eq. (2.11) as a system of coupled differential equations for the functions $u_{n}(t)$. Thus, in general we have infinitely many equations for infinitely many functions. In this case the Ansatz for the vector field $\widehat{X}$ would be:

$$
\widehat{X}=\tau\left(t,\left\{u_{j}(t)\right\}_{j}\right) \partial_{t}+\sum_{k} \phi_{k}\left(t,\left\{u_{j}(t)\right\}_{j}\right) \partial_{u_{k}(t)}
$$

where by $\left\{u_{j}(t)\right\}_{j}$ we mean the set of all $u_{j}(t)$ and $j$ and $k$ vary a priori over an infinite range. Calculating the second prolongation $\operatorname{pr}^{(2)} \widehat{X}$ in a standard manner ( see eqs. (1.7), (1.9) ) and imposing

$$
\left.\operatorname{pr}^{(2)} \widehat{X} \Delta_{n}^{(2)}\right|_{\Delta_{j}^{(2)}=0}=0 \quad \forall n, j
$$

we obtain, in general, an infinite system of determining equations for an infinite number of functions. Conceptually speaking, this second method, called the differential equation method in Ref. [151], may give rise to a larger symmetry group 
than the intrinsic method we introduced before. In fact the intrinsic method yields purely point transformations, while the differential equation method can yield generalized symmetries with respect to the differences (but not the derivatives). In practice, it turns out that usually no higher order symmetries with respect to the discrete variable exist; then the two methods give the same result and the intrinsic method is simpler.

A third approach $[200,201]$ consists of interpreting the variable $n$ as a continuous variable and consequently the $\mathrm{D} \Delta \mathrm{E}$ as a differential-delay equation. In such an approach $u_{n+k}(t) \equiv \exp \left[\frac{k \partial}{\partial n}\right]\left\{u_{n}(t)\right\}$ and consequently the $\mathrm{D} \Delta \mathrm{E}$ is interpreted as a partial differential equation of infinite order. In such a case formula (2.16) is meaningless as we are not able to construct the infinite order prolongation of a vector field $\widehat{X}$. The Lie symmetries are obtained by requiring that the solution set of the equation $\Delta_{n}^{(2)}=0$ (2.3) be invariant under the infinitesimal transformation

$$
\begin{aligned}
\tilde{t} & =t+\epsilon \tau_{n}\left(t, u_{n}(t)\right), \\
\tilde{n} & =n+\epsilon \nu_{n}\left(t, u_{n}(t)\right), \\
\tilde{u}_{\tilde{n}}(\tilde{t}) & =u_{n}(t)+\epsilon \phi_{n}\left(t, u_{n}(t)\right) .
\end{aligned}
$$

To obtain conditional symmetries we add to eq. (2.11) a constraining equation which we choose in such a way that it is automatically annihilated on its solution set by the prolongation of the vector field. Such an equation is the invariant surface condition

$$
\Delta_{n}^{(1)}=\phi_{n}\left(t, u_{n}(t)\right)-\tau_{n}\left(t, u_{n}(t)\right) u_{n, t}(t)=0 .
$$

In general eqs. 2.11 and 2.23) may not be compatible; if they are, then eq. (2.23) provides a reduction of the number of the independent variables by one. This is the essence of the symmetry reduction by conditional symmetries. Due to the fact that eq. (2.23) is written in terms of $\tau$ and $\phi$, which are the coefficients of the vector field $\widehat{X}$, the determining equations are nonlinear. The vector fields do not form an algebra, nor even a vector space, since each vector field is adapted to a different condition [22,41,147].

\subsection{Symmetries of the Toda lattice}

Let us now apply the techniques introduced in Section 2.2 to the case of eq. (2.3). In this case eq. (2.16) reduces to an overdetermined system of determining equations obtained by equating to zero the coefficients of $\left[v_{n, t}\right]^{k}, k=0,1,2,3$ and of $v_{n \pm 1}$. They imply:

$$
\tau=a t+d, \quad \phi=b+2 a n+c t, \quad a, b, c, d \text { real constants, }
$$

corresponding to a four dimensional Lie algebra generated by the vector fields:

$$
\widehat{D}=t \partial_{t}+2 n \partial_{v_{n}}, \quad \widehat{T}=\partial_{t}, \quad \widehat{W}=t \partial_{v_{n}}, \quad \widehat{U}=\partial_{v_{n}} .
$$


The group transformation which will leave eq. (2.3) invariant is hence:

$$
\tilde{v}_{n}(\tilde{t})=v_{n}\left(\tilde{t} e^{-\lambda_{4} / 2}-\lambda_{3}\right)+\lambda_{2}\left(\tilde{t} e^{-\lambda_{4} / 2}-\lambda_{3}\right)+\lambda_{4} n+\lambda_{1}
$$

where $\lambda_{j}, j=1,2,3,4$, are real group parameters.

To the transformation (2.26) we can add some discrete ones [136]:

$$
\tilde{n}=n+N \quad N \in \mathbb{Z}
$$

and

$$
\left(t, v_{n}\right) \rightarrow\left(-t, v_{n}\right) ; \quad\left(t, v_{n}\right) \rightarrow\left(t,-v_{-n}\right) .
$$

We write the symmetry group of eq. (2.3) as:

$$
\mathfrak{G}=\mathfrak{G}_{D} \triangleright \mathfrak{G}_{C}
$$

where $\mathfrak{G}_{D}$ are the discrete transformations (2.27), (2.28) and the invariant subgroup $\mathfrak{G}_{C}$ corresponds to the transformation (2.26). A complete classification of the one dimensional subgroups of $\mathfrak{G}$ can be easily obtained [151].

In fact, if we complement the Lie algebra (2.25) by the vector field

$$
\widehat{Z}=\frac{\partial}{\partial n}
$$

and require, at the end of the calculations, that the corresponding group parameter be integer, the commutation relations become:

$$
[\widehat{Z}, \widehat{D}]=2 \widehat{U} ; \quad[\widehat{D}, \widehat{T}]=-\widehat{T} ; \quad[\widehat{D}, \widehat{W}]=\widehat{W} ; \quad[\widehat{T}, \widehat{W}]=\widehat{U}
$$

The one dimensional subalgebras are:

$$
\begin{gathered}
\{\widehat{Z}+a \widehat{D}+b \widehat{U}\}, \quad\{\widehat{Z}+a \widehat{T}+k \widehat{W}\}, \quad\{\widehat{Z}+\epsilon \widehat{W}\}, \quad\{\widehat{Z}+a \widehat{U}\} \\
\{\widehat{T}+c \widehat{W}\}, \quad\{\widehat{D}+c \widehat{U}\}, \quad\{\widehat{U}\}, \quad\{\widehat{Z}\}, \quad\{\widehat{W}\}, \\
(a, b, c) \in \mathbb{R} ; a \neq \equiv ; k=0,1,-1 ; \epsilon= \pm 1 .
\end{gathered}
$$

Nontrivial solutions, corresponding to reductions with respect to continuous subgroups $\mathfrak{G}_{0} \subset \mathfrak{G}_{C}$, are obtained by considering invariance of the Toda lattice under $\{\widehat{T}+c \widehat{W}\}$, or $\{\widehat{D}+c \widehat{U}\}$. They are:

$$
\begin{aligned}
& v_{n}(t)=p-\frac{1}{2} c t^{2}-\sum_{j=1}^{n} \log (q-c j), \\
& v_{n}(t)=p+2(n+c) \log (t)-\sum_{j=0}^{n} \log \left[q+(2 c-1) j+j^{2}\right],
\end{aligned}
$$

where $p$ and $q$ are arbitrary integration parameters.

Reduction by the purely discrete subgroup, $\mathfrak{G}_{0} \subset \mathfrak{G}_{D}$, given in eq. (2.27) implies the invariance of eq. (2.3) under discrete translation of $n$ and makes it possible to impose the periodicity condition

$$
u(n+N, t)=u(n, t) .
$$


This reduces the $\mathrm{D} \Delta \mathrm{E}(2.3)$ to an ordinary differential equation (or a finite system of equations). For example for $N=2$, we get

$$
v_{, t t}=-4 \sinh v
$$

while for $N=3$, we get the Tzitzeica differential equation $[234,235]$ :

$$
v_{, t t}=e^{-2 v}-e^{v} .
$$

Let us now consider a subgroup $\mathfrak{G}_{0} \subset \mathfrak{G}$ that is not contained in $\mathfrak{G}_{C}$, nor in $\mathfrak{G}_{D}$, i.e. a nonsplitting subgroup of $\mathfrak{G}$. A reduction corresponding to $\widehat{Z}+a \widehat{D}+b \widehat{U}$ yields the equation

$$
F^{\prime \prime}(y)=e^{-b}\left[\exp \left(F\left(y e^{a}\right)-F(y)+a\right)-\exp \left(F(y)-F\left(y e^{-a}\right)-a\right)\right]
$$

where the symmetry variables $F(y)$ and $y$ are defined by:

$$
y=t e^{-a n}, \quad v_{n}(t)=a n^{2}+b n+F(y) .
$$

Using the subalgebra $\widehat{Z}+a \widehat{T}+k \widehat{W}$ we get

$$
F^{\prime \prime}(y)=e^{F(y+a)-F(y)}-e^{F(y)-F(y-a)}-\frac{k}{a},
$$

where

$$
y=t-a n, \quad v_{n}(t)=\frac{k}{2 a} t^{2}+F(y) .
$$

Eq. (2.38) can be called a "differential dilation" type equation; it involves one independent variable $y$, but the function $F$ and its derivatives are evaluated at the point $y$ and at the dilated points $y e^{a}$ and $y e^{-a}$. Eq. (2.40) is a differential delay equation which has interesting solutions, such as the soliton and periodic solutions of the Toda lattice (for $k=0$ ).

The other two nonsplitting subgroups give rise to linear delay equations which can be solved explicitely.

This same calculation can also be carried out for the inhomogeneous Toda lattice (2.13). The symmetry algebra is

$$
\begin{gathered}
\widetilde{D}=2 \partial_{\tilde{t}}+\frac{1}{2} \partial_{w_{n}}, \quad \widetilde{T}=e^{-\tilde{t} / 2}\left[\partial_{\tilde{t}}-\left(w_{n}-\frac{1}{2}\right) \partial_{w_{n}}\right] \\
\widetilde{W}=2 e^{\tilde{t} / 2} \partial_{w_{n}}, \quad \widetilde{U}=\partial_{w_{n}} .
\end{gathered}
$$

These vector fields have the same commutation relations as those of the Toda lattice (2.3). This is a necessary condition for the existence of a point transformation between the two equations. In fact by comparing the two sets of vector fields, we get the following transformation which transforms a solution $v_{n}(t)$ of equation (2.3) into a solution $w_{n}(\tilde{t})$ of eq. (2.13)

$$
\begin{gathered}
\tilde{t}=2 \log \left(\frac{t}{2}\right), \\
w_{n}(\tilde{t})=v_{n}(t)-2\left(n-\frac{1}{2}\right) \log (t)+\sum_{j=0}^{n}\left[\frac{1}{4}(j-1)^{2}+1\right] .
\end{gathered}
$$


Let us now calculate the conditional symmetries of the Toda lattice. We assume that $\tau$ is not zero and the determining equation reads:

$$
\begin{aligned}
\phi_{n, t t} & +\phi_{n, t} \phi_{n, v_{n}}+2 \phi_{n} \phi_{n, t v_{n}}+\phi_{n}\left[\phi_{n, v_{n}}^{2}+\phi_{n} \phi_{\left.n, v_{n} v_{n}\right)}\right] \\
& +\left[2 \phi_{n}-\phi_{n-1}-\phi_{n+1}\right] e^{v_{n}-v_{n+1}}+\left[\phi_{n}-\phi_{n-1}\right]\left[\phi_{n, t}-\phi_{n} \phi_{n, v_{n}}\right]=0 .
\end{aligned}
$$

This implies

$$
\phi_{n}\left(t, v_{n}(t)\right)=\alpha(t)+\beta(t) n
$$

with

$$
\beta_{, t t}+\beta \beta_{, t}=0 ; \quad \alpha_{, t t}+\beta \alpha_{, t}=0 .
$$

Solving eqs. (2.46) we get

$$
\phi_{n}\left(t, v_{n}(t)\right)= \begin{cases}K_{0}+\left(2 \frac{K_{1}}{K_{3}}+n K_{3}\right) \tanh \left[\frac{K_{3}}{2}\left(t-t_{0}\right)\right], & \text { for } K_{3} \neq 0 \\ \frac{K_{0} t+K_{1}+2 n}{t-t_{0}}, & \text { for } K_{3}=0 .\end{cases}
$$

For $K_{3}=0$ we get the results given in eq. (2.24). For $K_{3} \neq 0$ an additional "symmetry" is given by $\phi=K_{3} n \tanh \left(K_{3} t / 2\right)$. This gives a new explicit solution of the Toda lattice:

$$
v_{n}(t)=u_{0}+2 n K_{3} \log \left[\cosh \left(\frac{K_{3}}{2} t\right)\right] .
$$

\subsection{Classification of differential equations on a lattice}

Group theoretical methods can also be used to classify equations according to their symmetry groups. This has been done in the case of partial differential equations [78] showing, for instance, that in the class of variable coefficient Korteweg-de Vries equations, the Korteweg-de Vries itself has the largest sym-

metry group. The same kind of results can also be obtained in the case of differential-difference equations.

Let us consider a class of equations involving nearest neighbour interactions $[156]$

$$
\Delta_{n}=u_{n, t t}(t)-F_{n}\left(t, u_{n-1}(t), u_{n}(t), u_{n+1}(t)\right)=0,
$$

where $F_{n}$ is nonlinear in $u_{k}(t)$ and coupled, i.e. such that $F_{n, u_{k}} \not \equiv 0$ for some $k \not \equiv n$.

We consider point symmetries only; the continuous transformations of the form (2.14) are again generated by a Lie algebra of vector fields of the form 2.15). Taking into account the form of eq (2.49), we have

$$
\widehat{X}=\tau(t) \partial_{t}+\left[\left(\frac{1}{2} \tau_{, t}+a_{n}\right) u_{n}+b_{n}(t)\right] \partial_{u_{n}}
$$


with $a_{n, t}=0$. The determining equations reduce to

$$
\begin{aligned}
\frac{1}{2} \tau_{, t t t} u_{n}+b_{n, t t} & +\left(a_{n}-\frac{3}{2} \tau_{, t}\right) F_{n}-\tau F_{n, t} \\
& -\sum_{k=0, \pm 1}\left[\left(\frac{1}{2} \tau_{, t}+a_{n+k}\right) u_{n+k}+b_{n+k}(t)\right] F_{n, u_{n+k}}=0 .
\end{aligned}
$$

Our aim is to solve eq. (2.51) with respect to both the form of the nonlinear equation, i.e. $F_{n}$, and the symmetry vector field $\widehat{X}$, i.e. $\left(\tau(t), a_{n}, b_{n}(t)\right)$. In other words, for every nonlinear interaction $F_{n}$ we wish to find the corresponding maximal symmetry group $\mathfrak{G}$. Associated with any symmetry group $\mathfrak{G}$ there will be a whole class of nonlinear differential-difference equations related to each other by point transformations. To simplify the results, we will just look for the simplest element of a given class of nonlinear differential-difference equations, associated to a certain symmetry group. To do so we introduce so called allowed transformations, i.e. a set of transformations of the form

$$
\tilde{t}=\tilde{t}(t), \quad \tilde{n}=n \quad u_{n}(t)=\Omega_{n}\left(\tilde{u}_{n}(\tilde{t}), t\right)
$$

that transform equation (2.49) into a different one of the same type. By a straightforward calculation we find that the only allowed transformations (2.52) are given by

$$
\tilde{t}=\tilde{t}(t), \quad \tilde{n}=n, \quad u_{n}(t)=\frac{A_{n}}{\sqrt{\tilde{t}_{, t}(t)}} \tilde{u}_{n}(\tilde{t})+B_{n}(t)
$$

with $B_{n}(t), A_{n}, \tilde{t}(t)$ arbitrary functions of their arguments.

Under an allowed transformation equation (2.49) is transformed into

$$
\tilde{u}_{n, \tilde{t} \tilde{t}}(\tilde{t})=\widetilde{F}_{n}\left(n, \tilde{t}, \tilde{u}_{n+1}(\tilde{t}), \tilde{u}_{n}(\tilde{t}), \tilde{u}_{n-1}(\tilde{t})\right)
$$

with

$$
\begin{aligned}
\tilde{F}_{n}=\frac{1}{\tilde{t}_{, t}^{\frac{3}{2}} A_{n}}\left\{F_{n}\left(n, t,\left\{u_{n+1}(t), u_{n}(t), u_{n-1}(t)\right\}\right)-B_{n, t t}-\right. \\
\left.-\left[\frac{3}{4} \frac{\tilde{t}_{, t t}^{2}}{\tilde{t}_{, t}^{\frac{5}{2}}}-\frac{1}{2} \frac{\tilde{t}_{, t t t}}{\tilde{t}_{, t}^{\frac{3}{2}}}\right] A_{n} \tilde{u}_{n}(\tilde{t})\right\}
\end{aligned}
$$

and a symmetry generator (2.50) into

$$
\begin{aligned}
\widehat{\widetilde{X}}=\left[\tau(t) \tilde{t}_{, t}\right] \partial_{\tilde{t}}+\left\{\left[\frac{\tau(t)}{2} \frac{\tilde{t}_{, t t}}{\tilde{t}_{, t}}+\frac{1}{2} \tau_{, t}(t)+a_{n}\right] \tilde{u}_{n}+\right. \\
\left.+\frac{\tilde{t}_{, t}^{\frac{1}{2}}}{A_{n}}\left[-\tau(t) B_{n, t}(t)+B_{n}(t)\left(\frac{1}{2} \tau_{, t}(t)+a_{n}\right)+b_{n}(t)\right]\right\} \partial_{\tilde{u}_{n}} .
\end{aligned}
$$


We see that, up to an allowed transformation, every one-dimensional symmetry algebra associated to eq. (2.49), can be represented by one of the following vector fields:

$$
\widehat{X}_{1}=\partial_{t}+a_{n}^{1} u_{n} \partial_{u_{n}} \quad \widehat{X}_{2}=a_{n}^{2} u_{n} \partial_{u_{n}} \quad \widehat{X}_{3}=b_{n}(t) \partial_{u_{n}}
$$

where $a_{n}^{j}$ with $j=1,2$ are two arbitrary functions of $n$ and $b_{n}(t)$ is an arbitrary function of $n$ and $t$. The vector fields $\widehat{X}_{j}, j=1,2,3$ are the symmetry vectors of the Lie point symmetries of the following nonlinear differential difference equations:

$$
\begin{array}{llll}
\widehat{X}_{1}: & u_{n, t t}=e^{a_{n}^{1} t} f_{n}\left(\xi_{n+1}, \xi_{n}, \xi_{n-1}\right), & & \text { with } \xi_{j}=u_{j} e^{-a_{j}^{1} t} \\
\widehat{X}_{2}: & u_{n, t t}=u_{n} f_{n}\left(t, \eta_{n+1}, \eta_{n-1}\right), & & \text { with } \eta_{j}=\frac{\left(u_{j}\right)^{a_{n}^{2}}}{\left(u_{n}\right)^{a_{j}^{2}}} \\
\widehat{X}_{3}: & u_{n, t t}=\frac{b_{n, t t}}{b_{n}} u_{n}+f_{n}\left(t, \zeta_{n+1}, \zeta_{n-1}\right) & & \text { with } \zeta_{j}=u_{j} b_{n}(t)-u_{n} b_{j}(t) .
\end{array}
$$

These equations are still quite general, as they are written in terms of arbitrary functions depending on three continuous variables. More specific equations are obtained for larger symmetry groups [156].

The Toda equation (2.3) is included in a class of equations whose infinitesimal symmetry generators satisfy a four dimensional solvable symmetry algebra with a nonabelian nilradical. The interactions in this class are given by

$$
F_{n}\left(t, u_{n-1}(t), u_{n}(t), u_{n+1}(t)\right)=e^{-2 \frac{u_{n+1}-u_{n}}{\gamma_{n+1}-\gamma_{n}}} f_{n}(\xi)
$$

where $\xi=\left(\gamma_{n}(t)-\gamma_{n+1}(t)\right) u_{n-1}+\left(\gamma_{n+1}(t)-\gamma_{n-1}(t)\right) u_{n}+\left(\gamma_{n-1}(t)-\gamma_{n}(t)\right) u_{n+1}$ and the function $\gamma_{n}(t)$ is such that $\gamma_{n+1}(t) \neq \gamma_{n}(t)$ and $\frac{\partial \gamma_{n}(t)}{\partial t}=0$. The associated symmetry generators are:

$$
\widehat{X}_{1}=\partial_{u_{n}}, \quad \widehat{X}_{2}=\partial_{t}, \quad \widehat{X}_{3}=t \partial_{u_{n}}, \quad \widehat{Y}=t \partial_{t}+\gamma_{n}(t) \partial_{u_{n}} .
$$

The Toda equation (2.3) is obtained by choosing $\gamma_{n}(t)=2 n$ and $f_{n}(\xi)=-1+$ $e^{\frac{1}{2} \xi}$. Among the equations of the class (2.49), the Toda equation does not have the largest group of point symmetries.

A complete list of all equations of the type (2.49) with nontrivial symmetry group is given in the original article [156] with the additional assumption that the interaction and the vector fields depend continuously on $n$. Here we just give two examples of interactions with symmetry groups with dimension seven. The first one is solvable, nonnilpotent and its Lie algebra is given by

$$
\begin{gathered}
\widehat{X}_{1}=\partial_{u_{n}}, \quad \widehat{X}_{2}=(-1)^{n} \partial_{u_{n}}, \quad \widehat{X}_{3}=t \partial_{u_{n}} \\
\widehat{X}_{4}=(-1)^{n} t \partial_{u-n}, \quad \widehat{X}_{5}=(-1)^{n} u_{n} \partial_{u_{n}}, \quad \widehat{X}_{6}=\partial_{t}, \quad \widehat{X}_{7}=t \partial_{t}+2 u_{n} \partial_{u_{n}} .
\end{gathered}
$$

The invariant equation is

$$
u_{n, t t}=\frac{\gamma_{n}}{u_{n-1}-u_{n+1}}
$$


This algebra was not included in Ref [156] because of its nonanalytical dependence on $n$ (in $\hat{X}_{2}, \hat{X}_{4}$ and $\hat{X}_{5}$ ).

The second symmetry algebra is nonsolvable; it contains the simple Lie algebra $\operatorname{sl}(2, \mathbb{R})$ as a subalgebra. A basis of this algebra is

$$
\begin{gathered}
\widehat{X}_{1}=\partial_{u_{n}}, \quad \widehat{X}_{2}=t \partial_{u_{n}}, \quad \widehat{X}_{3}=b_{n} \partial_{u_{n}} \\
\widehat{X}_{4}=b_{n} t \partial_{u_{n}}, \quad \widehat{X}_{5}=\partial_{t}, \quad \widehat{X}_{6}=t \partial_{t}+\frac{1}{2} u_{n} \partial_{u_{n}}, \quad \widehat{X}_{7}=t^{2} \partial_{t}+t u_{n} \partial_{u_{n}}
\end{gathered}
$$

with $b_{n, t}=0, b_{n+1} \neq b_{n}$. The corresponding invariant nonlinear differential equation is:

$$
u_{n, t t}=\frac{\gamma_{n}}{\left[\left(b_{n+1}-b_{n}\right) u_{n-1}+\left(b_{n-1}-b_{n+1}\right) u_{n}+\left(b_{n}-b_{n-1}\right) u_{n+1}\right]^{3}}
$$

where $\gamma_{n}$ and $b_{n}$ are arbitrary $n$-dependent constants.

In [240] the integrability conditions for equations belonging to the class (2.49) has been considered. It has been shown that any equation of this class which has local generalized symmetries can be reduced by point transformations of the form

$$
\tilde{u}_{n}=\sigma_{n}\left(t, u_{n}\right), \quad \tilde{t}=\theta(t)
$$

to either the Toda equation (2.3) or to the potential Toda equation

$$
u_{n, t t}=e^{u_{n+1}-2 u_{n}+u_{n-1}} .
$$

\subsection{Symmetries of the Two Dimensional Toda equation}

Let us now apply the techniques introduced in Section 2.2 to the two-dimensional Toda system (TDTS)

$$
\Delta_{T D T S}=u_{n, x t}-e^{u_{n-1}-u_{n}}+e^{u_{n}-u_{n+1}}=0
$$

where $u_{n}$ is also a function of $t$ and $x$. The TDTS was proposed and studied by Mikhailov [178] and Fordy and Gibbons [66] and is an integrable D $\Delta \mathrm{E}$, having a Lax pair, infinitely many conservation laws, Bäcklund transformations, soliton solutions, and all the usual attributes of integrability $[1,8,33]$.

The continuous symmetries for eq. (2.67) are obtained by considering the infinitesimal symmetry generator

$$
\hat{X}=\xi_{n}\left(x, t, u_{n}\right) \partial_{x}+\tau_{n}\left(x, t, u_{n}\right) \partial_{t}+\phi_{n}\left(x, t, u_{n}\right) \partial_{u_{n}} .
$$

From the determining equation (2.16) we get

$$
\tau_{n}=f(t), \quad \xi_{n}=h(x) . \quad \phi_{n}=\left(h_{, x}+f_{, t}\right) n+g(t)+k(x),
$$

where $f(t), g(t), h(x)$ and $k(x)$ are arbitrary $C^{\infty}$ functions. A basis for the symmetry algebra is given by

$$
\begin{gathered}
T(f)=f(t) \partial_{t}+n f_{, t} \partial_{u_{n}}, \quad U(g)=g(t) \partial_{u_{n}} \\
X(h)=h(x) \partial_{x}+n h_{, x} \partial_{u_{n}}, \quad W(k)=k(x) \partial_{u_{n}}
\end{gathered}
$$


where, to avoid redundancy, we must impose $k_{, x} \neq 0$.

The nonzero commutation relations are

$$
\begin{aligned}
& {\left[T\left(f_{1}\right), T\left(f_{2}\right)\right]=T\left(f_{1} f_{2, t}-f_{1, t} f_{2}\right), \quad[T(f), U(g)]=U\left(f g_{, t}\right),} \\
& {\left[X\left(h_{1}\right), X\left(h_{2}\right)\right]=X\left(h_{1} h_{2, x}-h_{1, x} h_{2}\right),} \\
& {[X(h), W(k)]=\left\{\begin{array}{cc}
W\left(h k_{, x}\right), \quad\left(h k_{, x}\right)_{, x} \neq 0 \\
c U(1), \quad\left(h k_{, x}\right)_{, x}=0, \quad h k_{, x}=c .
\end{array}\right.}
\end{aligned}
$$

Thus $\{T(f), U(g)\}$ form a Kac-Moody-Virasoro $\hat{u}(1)$ algebra, as do $\{X(h)$, $\left.W(k), h_{, x} \neq 0, U(1)\right\}$. However the two $\hat{u}(1)$ algebras are not disjoint. This Kac-Moody-Virasoro character of the symmetry algebra is found also in the case of a $(2+1)$-dimensional Volterra equation. It is also characteristic of many other integrable equations involving three continuous variables, such as the Kadomtsev-Petviashvili or three-wave equations [36, 45, 46, 146, 177, 197]. From the symmetry algebra we can construct the group of symmetry transformations which leave the TDTS (2.67) invariant and transform noninvariant solutions into new solutions. Moreover, we can use the subgroups to reduce the TDTS (2.67) to equations in a lower dimensional space.

By adding to (2.67) the equation

$$
\Delta_{n}^{(1)}=\xi_{n}\left(x, t, u_{n}\right) u_{n, x}+\tau_{n}\left(x, t, u_{n}\right) u_{n, t}-\phi_{n}\left(x, t, u_{n}\right)=0
$$

we obtain the conditional symmetries. It is easy to show that conditional symmetries in the intrinsic method do not provide any further symmetry reduction. It is, however, worthwhile to notice that conditional symmetries in the differential equation method, when

$$
\hat{X}=\xi_{n}\left(x, t, u_{j}(x, t)\right) \partial_{x}+\tau_{n}\left(x, t, u_{j}(x, t)\right) \partial_{t}+\sum_{k} \phi_{k}\left(x, t, u_{j}(x, t)\right) \partial_{u_{k}},
$$

contain the Bäcklund transformation of the TDTS. In fact, by choosing $\xi_{n}=0$, $\tau_{n}=1$, eq. (2.73) reduces to $\Delta_{n}^{(1)}=u_{n, t}-\phi_{n}\left(x, t, u_{j}\right)=0$ and the determining equations are solved by putting

$$
\phi_{n}\left(x, t, u_{j}\right)=f_{n, t}(x, t)+a\left[e^{u_{n}-f_{n+1}}-e^{u_{n-1}-f_{n}}\right]
$$

where $a$ is a real constant and $f_{n}(x, t)=\tilde{u}_{n}$ is a set of functions which solves the TDTS (2.67). The Bäcklund transformation for the TDTS can be indeed written as [66]

$$
\begin{aligned}
u_{n, t}-\tilde{u}_{n, t} & =a\left[e^{u_{n}-\tilde{u}_{n+1}}-e^{u_{n-1}-\tilde{u}_{n}}\right], \\
u_{n-1, x}-\tilde{u}_{n, x} & =\frac{1}{a}\left[e^{\tilde{u}_{n-1}-u_{n-1}}-e^{\tilde{u}_{n}-u_{n}}\right] .
\end{aligned}
$$

\section{Generalized Point Symmetries on a Fixed Uni- form Lattice}

We saw in Section 2 that point symmetries for purely difference equations on a fixed uniform lattice do not provide very powerful tools, though they work well for differential - difference equations. 
Here we shall consider an approach that makes use of a certain type of generalized symmetries that act simultaneously at more than one point of the lattice. We call them "generalized point symmetries", because in the continuous limit they reduce to point symmetries.

This approach is directly applicable to linear difference equations, indirectly to nonlinear equations that are linearizable by a change of variables.

The underlying formalism is called "umbral calculus", or "finite operator calculus". Its modern development is mainly due to G.C. Rota and his collaborators [210-212]. For a review article with an extensive list of references, see [47]. Umbral calculus has been implicitly used in mathematical physics $[63,64,82,128,130,145]$. The only explicit use in physics that we know of is in $[37,48,139,140]$.

\subsection{Basic Concepts of Umbral Calculus}

Definition 1. A shift operator $T_{\sigma}$ is a linear operator acting on polynomials or formal power series $f(x)$ in the following manner

$$
T_{\sigma} f(x)=f(x+\sigma), \quad x \in \mathbb{R}, \quad \sigma \in \mathbb{R} .
$$

For functions of several variables we introduce shift operators in the same manner

$$
\begin{aligned}
T_{\sigma_{i}} f\left(x_{1}, \ldots x_{i-1}, x_{i}, x_{i+1} \ldots x_{n}\right) & \\
& =f\left(x_{1}, \ldots, x_{i-1}, x_{i}+\sigma_{i}, x_{i+1}, \ldots, x_{n}\right) .
\end{aligned}
$$

In this section we restrict the exposition to the case of one real variable $x \in \mathbb{R}$. The extension to $n$ variables and other fields is obvious. We will sometimes drop the subscript on the shift operator $T$ when that does not give rise to misinterpretations.

Definition 2. An operator $U$ is called a delta operator if it satisfies the following properties,

1) It is shift invariant;

$$
T_{\sigma} U=U T_{\sigma}, \quad \forall \sigma \in \mathbb{R},
$$

$$
U x=c \neq 0, \quad c=\text { const }
$$

3)

$$
U a=0, \quad a=\text { const, },
$$

and the kernel of $U$ consists precisely of constants.

Important properties of delta operators are:

1. For every delta operator $U$ there exists a unique series of basic polynomials $\left\{P_{n}(x)\right\}$ satisfying

$$
P_{0}(x)=1 \quad P_{n}(0)=0, \quad n \geq 1, \quad U P_{n}(x)=n P_{n-1}(x) .
$$


2. For every delta operator $U$ there exists a conjugate operator $\beta$, such that

$$
[U, x \beta]=1 .
$$

The operator $\beta$ satisfies

$$
\beta=\left(U^{\prime}\right)^{-1}, \quad U^{\prime}=[U, x] .
$$

Eq. (3.7) can be interpreted as the Heisenberg relation between the delta operator $U$ and its conjugate $x \beta$.

We shall make use of two types of delta operators. The first is the ordinary (continuous) derivative operator, for which we have

$$
U=\partial_{x}, \quad \beta=1, \quad P_{n}(x)=x^{n} .
$$

The second is a general difference operator which we define as

$$
U=\Delta=\frac{1}{\sigma} \sum_{k=l}^{m} a_{k} T_{\sigma}^{k}, \quad l, m \in \mathbb{Z}, \quad l<m,
$$

where $a_{k}$ and $\sigma$ are real constants and $T_{\sigma}$ is a shift operator. In order for $\Delta$ to be a delta operator, we must impose

$$
\sum_{k=l}^{m} a_{k}=0 .
$$

We shall also require that the continuous limit of $\Delta$ be $\partial_{x}$. This imposes a further condition, namely

$$
\sum_{k=l}^{m} k a_{k}=1 .
$$

Than eqs. (3.3), .., 3.5 hold, with $c=1$.

If eqs. (3.11), (3.12) are satisfied, we shall call $\Delta$ a difference operator of order $m-l$. Acting with $\Delta$ on an arbitrary smooth function $f(x)$ we have

$$
\begin{aligned}
\Delta f(x) & =\frac{1}{\sigma} \sum_{k=l}^{m} a_{k} f(x+k \sigma)=\frac{1}{\sigma} \sum_{n=0}^{\infty} \frac{f^{(n)}(x)}{n !} \sigma^{n} \gamma_{n}, \\
\gamma_{n} & =\sum_{k=l}^{m} a_{k} k^{n}, \quad \gamma_{0}=0, \gamma_{1}=1 .
\end{aligned}
$$

Thus we have

$$
\Delta f(x)=\frac{\partial f}{\partial x}+\sum_{n=2}^{\infty} \frac{\left.f^{(n}\right)(x)}{n !} \sigma^{n-1} \gamma_{n} .
$$

For $m-l \geq 2$ we can impose further conditions, namely

$$
\gamma_{n}=0, \quad n=2,3, \ldots, m-l,
$$


and obtain

$$
\Delta=\frac{d}{d x}+O\left(\sigma^{m-l}\right) .
$$

From (3.8) the operator $\beta$ conjugate to $\Delta$ is

$$
\beta=\left(\sum_{k=l}^{m} a_{k} k T_{\sigma}^{k}\right)^{-1}
$$

and the basic polynomials are

$$
P_{n}(x)=(x \beta)^{n} 1
$$

It was shown in [139] that the eq. 3.18) yields a well defined polynomial of order $n$ in $x$. For any differential operator $\Delta$ all coefficients in $P_{n}(x)$ are finite and each involves a finite number of positive powers of shifts in $\sigma$.

The simplest examples of difference operators and the related quantities are

1.

$$
\begin{aligned}
\Delta^{+} & =\frac{T-1}{\sigma}, \quad \beta=T^{-1} \\
P_{n}(x) & =(x)_{n}=x(x-\sigma)(x-2 \sigma) \ldots(x-(n-1) \sigma), \quad n \geq 1
\end{aligned}
$$

Order $m-l=1$.

2.

$$
\begin{aligned}
\Delta^{-} & =\frac{1-T^{-1}}{\sigma}, \quad \beta=T, \\
P_{n}(x) & =(x)^{n}=x(x+\sigma)(x+2 \sigma) \ldots(x+(n-1) \sigma), \quad n \geq 1
\end{aligned}
$$

Order $m-l=1$.

3.

$$
\begin{array}{r}
\Delta^{s}=\frac{T-T^{-1}}{2 \sigma}, \quad \beta=\left(\frac{T+T^{-1}}{2}\right)^{-1}, \\
P_{2 n}(x)=x^{2}\left(x^{2}-4 \sigma^{2}\right) \ldots\left(x^{2}-(2 n-2)^{2} \sigma^{2}\right), \quad n \geq 1, \\
P_{2 n+1}(x)=x\left(x^{2}-\sigma^{2}\right) \ldots\left(x^{2}-(2 n-1)^{2} \sigma^{2}\right), \quad n \geq 1 .
\end{array}
$$

Order $m-l=2$.

For any $\Delta$ we have $P_{0}=1$ and $P_{1}=x$.

An important tool in the umbral calculus is the umbral correspondence. This is a bijective mapping $M$ between two delta operators $U_{1}$ and $U_{2}$. This will induce a mapping between the corresponding operators $\beta_{1}$ and $\beta_{2}$, and also between the corresponding basic polynomials:

$$
U_{1} \stackrel{M}{\longleftrightarrow} U_{2}, \quad \beta_{1} \stackrel{M}{\longleftrightarrow} \beta_{2}, \quad P_{n}^{(1)} \stackrel{M}{\longleftrightarrow} P_{n}^{(2)} .
$$


Let us now consider linear operators $\hat{L}(x \beta, U)$ that are polynomials, or formal power series in $x \beta$ and $U$. Since the umbral correspondence preserves the Heisenberg commutation relation (3.7), it will also preserve commutation relations between the operators $\hat{L}(x \beta, U)$. In particular, we can take

$$
U_{1}=\partial_{x}, \quad \beta_{1}=1, \quad U_{2}=\Delta, \quad \beta_{2}=\beta,
$$

where $\Delta$ is any one of the difference operators (3.10) and $\beta$ is as in (3.17).

Let $A_{1}$ be a Lie algebra of vector fields of the form

$$
\widehat{X}_{\alpha}=\sum_{j=1}^{n} a_{\alpha}^{j}\left(x_{1}, \cdots, x_{n}\right) \partial_{x_{j}}, \quad\left[\widehat{X}_{\alpha}, \widehat{X}_{\beta}\right]=c_{\alpha \beta}^{\gamma} \widehat{X}_{\gamma} .
$$

The umbral correspondence will map this algebra onto an isomorphic Lie algebra $A_{2}$ of difference operators

$$
\widehat{X}_{\alpha}^{u}=\sum_{j=1}^{n} a_{\alpha}^{j}\left(x_{1} \beta_{1}, \cdots, x_{n} \beta_{n}\right) \Delta_{x_{j}} .
$$

\subsection{Umbral Calculus and Symmetries of Linear Difference Equations}

Lie point symmetries of linear differential equations can be expressed in terms of commuting linear operators. Indeed, let us consider a linear differential equation

$$
L u=0
$$

where $L$ is some linear differential operator. The Lie point symmetry algebra of this equation can be realized by evolutionary vector fields of the form (1.12), satisfying eq. (1.14). If (3.26) is an ordinary differential equation of order 3 or higher, or a partial differential equation of order 2 or higher, then the characteristic $Q$ of the vector field (1.12) will have a specific form

$$
Q=f(\vec{x})+\widehat{X} u,
$$

where $f(\vec{x})$ is the general solution of eq. (3.26) and $\widehat{X}$ is a first order linear operator of the form [20]

$$
\widehat{X}=\sum_{i=1}^{n} \xi_{i}(\vec{x}) \partial_{x_{i}}-\phi(\vec{x}),
$$

satisfying

$$
\left.[L, \widehat{X}] u\right|_{L u=0}=0 .
$$

Using the umbral correspondence, we can carry this result over to a class of linear difference equations. Indeed, let us associate a difference operator $L_{D}$ 
with $L$ by the umbral correspondence $\partial_{x_{i}} \rightarrow \Delta_{x_{i}}, x_{i} \rightarrow x_{i} \beta_{i}$. Eq. (3.26) is replaced by a difference equation

$$
L_{D} u=0 .
$$

The analogue of eq. (3.29) will hold, namely

$$
\left.\left[L_{D}, \widehat{X}_{D}\right] u\right|_{L_{D} u=0}=0 .
$$

The difference operator $\widehat{X}_{D}$ will not generate point transformations taking solutions into solutions (they cannot be integrated to point transformation in general). They do, however, provide commuting flows, i.e. difference equations compatible with eq. (3.30).

\subsection{Symmetries of the Time Dependent Schrödinger Equa- tion on a Lattice}

As an example let us apply the umbral correspondence to the free time - dependent Schrödinger equation. We obtain the difference equation

$$
L_{D} \psi=0, \quad L_{D}=i \Delta_{t}+\frac{1}{2} \sum_{k=1}^{n} \Delta_{x_{k} x_{k}} .
$$

The symmetries of eq. (3.32 will be represented in terms of the "discrete" evolutionary vector fields

$$
\widehat{X}_{D}^{E}=Q_{D} \partial_{\psi}+Q_{D}^{*} \partial_{\psi^{*}}, \quad Q_{D}=\eta_{D}-\tau_{D} \Delta_{t} \psi-\xi_{D}^{k} \Delta_{x_{k}} \psi,
$$

where $\eta_{D}, \tau_{D}$ and $\xi_{D}^{k}$ are functions of $t \beta_{t}, x_{j} \beta_{j}$ and $\psi, \psi^{*}$ (the $*$ indicates complex conjugation).

As in the continuous case, it can be shown [139], that the characteristic $Q$ will in this case have the form

$$
Q_{D}=\chi\left(x_{k} \beta_{k}, t \beta_{t}\right)+i \widehat{X}_{D} \psi
$$

where $\chi$ is the general solution of eq. (3.32). The first order difference operator $\widehat{X}_{D}$ satisfies

$$
\begin{aligned}
& \widehat{X}_{D}=i\left[\tau_{D}\left(t \beta_{t}\right) \Delta_{t}+\sum_{k=1}^{n} \xi_{D}^{k} \Delta_{x_{k}}-i \eta_{D}\right] \\
& {\left.\left[L_{D}, \widehat{X}_{D}\right] \psi\right|_{L_{D} \psi=0}=0}
\end{aligned}
$$

Explicitating and solving eq. (3.36), we obtain a difference realization of the Schrödinger algebra, first obtained in the continuous case by Niederer [183]. A 
basis for this algebra is given by the following operators:

$$
\begin{aligned}
& \hat{P}_{0}=\Delta_{t}, \quad \hat{P}_{j}=\Delta_{x_{j}}, \quad \hat{L}_{j, k}=\left(x_{j} \beta_{j}\right) \Delta_{x_{k}}-\left(x_{k} \beta_{k}\right) \Delta_{x_{j}}, \\
& \hat{B}_{k}=\left(t \beta_{t}\right) \Delta_{x_{k}}-\frac{i}{2} x_{k} \beta_{k}, \quad \hat{D}=2\left(t \beta_{t}\right) \Delta_{t}-\sum_{k=1}^{n}\left(x_{k} \beta_{k}\right) \Delta_{x_{k}}+\frac{1}{2}, \\
& \hat{C}=\left(t \beta_{t}\right)^{2} \Delta_{t}+\sum_{k=1}^{n}\left(t \beta_{t}\right)\left(x_{k} \beta_{k}\right) \Delta_{x_{k}}+\frac{1}{2} t \beta_{t}-\frac{i n}{4} \sum_{k=1}^{n}\left(x_{k} \beta_{k}\right)^{2}, \quad \hat{W}=i .
\end{aligned}
$$

Comparing with the continuous limit (or using the umbral correspondence), we see that $P_{0}, P_{j}$ correspond to time and space translation, $L_{j, k}$ to rotations, $B_{k}$ to Galilei boosts, $D$ and $C$ to dilations and projective transformations and $W$ to changes of phase of the wave function.

\subsection{Symmetries of the Discrete Heat Equation}

As a further example let us consider the discrete heat equation

$$
\Delta_{x x} u(x, t)-\Delta_{t} u(x, t)=0 .
$$

Eq. (3.38) is a linear partial difference equation on a two dimensional lattice.

Floreanini, Negro, Nieto and Vinet showed in [63] that (3.38) has a group of symmetries isomorphous to that of the continuous heat equation

$$
u_{, x x}(x, t)-u_{, t}(x, t)=0 .
$$

This result can be easily recovered using the umbral calculus $[128,145]$.

Since (3.38) is linear, the symmetries are obtained by considering an evolutionary vector field of the form

$$
\hat{X}_{e}=Q \partial_{u}=\left(\tau \Delta_{t}+\xi \Delta_{x}+f\right) u \partial_{u}
$$

and the determining equation is give by

$$
\Delta_{t} Q-\left.\Delta_{x x} Q\right|_{\Delta_{x x} u=\Delta_{t} u}=0
$$

whose explicit expression is

$$
\Delta_{t}\left(\xi \Delta_{x} u+\tau \Delta_{t} u+f u\right)-\left[\left.\Delta_{x x}\left(\xi \Delta_{x} u+\tau \Delta_{t} u+f u\right)\right|_{\Delta_{x x} u=\Delta_{t} u}=0 .\right.
$$

Defining $D_{x} f=[\Delta, f]$ it is immediate to see that $\left(D_{x} f\right) 1=(\Delta f) 1$ and for the operator $D$ the Leibnitz's rule is satisfied: $D_{x} f g=\left(D_{x} f\right) g+f\left(D_{x} g\right)$. In term of the operator $D$ we can split eq. (3.42) into the following overdetermined system of equations

$$
\begin{array}{ccrl}
D_{x} \tau=0, & D_{t} \tau-2 D_{x} \xi & =0 \\
D_{t} \xi-D_{x x} \xi-2 D_{x} f=0, & D_{t} f-D_{x x} f & =0 .
\end{array}
$$


From the umbral correspondence the solution of eq. (3.43) is

$$
\begin{aligned}
\tau & =\tau_{2}\left(t \beta_{t}\right)^{2}+\tau_{1} t \beta_{t}+\tau_{0} \\
\xi & =\frac{1}{2}\left(\tau_{1}+2 \tau_{2} t \beta_{t}\right) x \beta_{x}+\xi_{1} t \beta_{t}+\xi_{0} \\
f & =\tau_{2}\left[\frac{1}{4}\left(x \beta_{x}\right)^{2}+\frac{1}{2} t \beta_{t}\right]+\frac{1}{2} \xi_{1} x \beta_{x}+f_{0}
\end{aligned}
$$

where $\tau_{0}, \tau_{1}, \tau_{2}, \xi_{1}, \xi_{0}$ and $f_{0}$ are arbitrary constants, functions of the lattice spacing and shifts. By a suitable choice of these constants, we get the following representation of the symmetries

$$
\begin{aligned}
& \hat{P}_{0}=\left(\Delta_{t} u\right) \partial_{u}, \quad \hat{P}_{1}=\left(\Delta_{x} u\right) \partial_{u} \\
& \hat{W}=u \partial_{u}, \quad \hat{B}=\left[2 t \beta_{t} \Delta_{x} u+x \beta_{x} u\right] \partial_{u} \\
& \hat{D}=\left[2 t \beta_{t} \Delta_{t} u+x \beta_{x} \Delta_{x} u+\frac{1}{2} u\right] \partial_{u} \\
& \hat{K}=\left[\left(t \beta_{t}\right)^{2} \Delta_{t} u+t \beta_{t} x \beta_{x} \Delta_{x} u+\left(\frac{1}{4}\left(x \beta_{x}\right)^{2}+\frac{1}{2} t \beta_{t}\right) u\right] \partial_{u} .
\end{aligned}
$$

For any choice of the $\Delta$ and the corresponding $\beta$ operators we have a different representation of the symmetry algebra of the heat equation.

\section{Generalized Symmetries on Fixed Uniform Lat- tices}

\subsection{Generalized symmetries of difference equations}

We consider here the construction of generalized symmetries for difference equations and show the structure of the infinite dimensional Lie algebra of point and higher symmetries.

Generalized symmetries are symmetries whose infinitesimal generators depend on more than one point of the lattice and on derivatives with respect to the variables which vary in a continuous way. E. Noether was the first to notice in 1918 [191] that one can extend symmetries by including higher derivatives of the dependent variables in the transformation. They are more rare than point symmetries. However, we can obtain an infinite number of generalized symmetries [194] when the system is integrable $[1,8,33,59,182,192,241]$, i.e when the equations can be written as the compatibility condition for an overdetermined system of linear equations (the Lax pair) and can be linearized either directly or via an Inverse Scattering Transform.

Lie symmetries, both point and generalized [194], can be obtained as flows commuting with the equation at study. We introduce here just the minimal notions on integrability necessary to be able to construct the commuting flows. For more on integrability see $[1,8,33,59,182,192,230,231,241]$.

A nonlinear partial differential equation for one function of two variables

$$
E\left(x, t, u(x, t), u_{x}(x, t), u_{t}(x, t), \ldots\right)=0
$$


is said to be integrable if it can be characterized by a Lax pair [121]

$$
\begin{gathered}
L(u) \psi=\lambda \psi, \\
\psi_{t}=-M(u) \psi,
\end{gathered}
$$

a system of linear equations compatible only on the solution set of solutions of eq. (4.1). In eqs. (4.2 4.3) $\lambda$ is an eigenvalue, $L$ and $M$ are two linear operators in $\mathrm{x}$ with coefficients depending on $u$ but not on $\lambda$. The function $\psi$, often called the wave or spectral function, depends on the independent variables $(x, t)$ and on $\lambda$. If $\lambda_{t}=0$ than $\lambda$ is an integral of motion, together with all the functions which depend only on it. In the particular case when eq. (4.2) represents the Schrödinger Spectral Problem

$$
\psi_{, x x}+[\lambda-u] \psi=0, \quad \lambda=k^{2},
$$

for $u(x)$ vanishing at infinity, the solution of eq. (4.4) has the following asymptotic behaviour:

$$
\begin{aligned}
& \psi(x, k) \rightarrow b(k) e^{i k x}+a(k) e^{-i k x}, \quad(x \rightarrow+\infty), \\
& \psi(x, k) \rightarrow \quad e^{-i k x}, \quad(x \rightarrow-\infty) .
\end{aligned}
$$

It can be easily proved that, when $u(x, t)$ evolves according to the KdV equation [33], the function $a(k)$ is conserved, i.e. $\dot{a}(k)=0$. Thus, an infinite set of conservation laws can be obtained by expanding the transmission coefficient $1 / a(\lambda)$ in powers of $\lambda$.

The compatibility of eqs.4.2 4.3) implies the Lax equation

$$
L_{t}(u)=[L(u), M(u)]
$$

if $\lambda_{t}=0$ or

$$
L_{t}(u)=[L(u), M(u)]+f(L(u), t)
$$

for $\lambda_{t}=f(\lambda, t)$. Here $f(z, t)$ is an entire function of its first argument.

Eq. (4.7) or eq. (4.8) is equivalent to the nonlinear partial differential equation (4.1). In the first case we say that the equation (4.1) is obtained as an isospectral deformation of (4.2) while in the second case by a non-isospectral deformation. For isospectral deformations the existence of a Lax pair implies the existence of an infinite number of conserved quantities while in the nonisospectral case these quantities will have a prescribed evolution in time.

The solution of the Cauchy problem for these systems is obtained by solving the Inverse Scattering Problem [1,8,33]. The integrability of the isospectral systems has also been confirmed by the Painlevé test, by deriving the global properties of its solutions through singularity analysis [117]. There is strong evidence, supported by many results, that all integrable equations have the Painlevé property, i.e. all solutions are single-valued around all movable singularities. In the case of ordinary differential equations this idea was used by Kowalevski $[115,116]$ to identify integrable rotating tops. For partial differential equations it lead to the Ablowitz, Ramani and Segur conjecture $[6,7]$ 
which states that any ordinary differential equation which arises as a (symmetry) reduction of an integrable partial differential equation possesses the Painlevé property, possibly after a transformation of variables. This conjecture has never been proven but there are no known counterexamples.

Each integrable nonlinear partial differential equation (4.1), be it isospectral, or non-isospectral, is characterized by an operator $M(u)$, defined up to an arbitrary constant matrix. To each linear problem (4.2) we can associate a recursion operator $\mathcal{L}$ and thus a hierarchy of nonlinear partial differential equations [33]. The operators $M(u)$ appear in a sequence constructed in terms of $\mathcal{L}$. The construction of $\mathcal{L}$ from the linear problem (4.2) is algorithmic [3,28,29,32]. The solution of the Cauchy problem for the integrable equation (4.1) is obtained by performing the Spectral Transform and solving the Inverse Spectral Problem for the linear problem (4.2). Starting from a given initial potential $u(x, t=0)$ we can characterize the spectrum $S[u]$ of eq. (4.2). Solving the linear equation for the spectrum we can obtain the potential $u(x, t)$ for any $t>0$ by solving the inverse problem. Each equation of the hierarchy

$$
E_{j}\left(\left(x, t, u(x, t), u_{x}(x, t), u_{t}(x, t), \ldots\right)=0\right.
$$

is characterized by a different linear evolution of its spectrum $\left.S_{j}[u)\right]$. At the level of the spectrum, it is easy to prove that from the hierarchy of equations associated to a given linear equation (4.2) we can obtain a set of commuting flows. Thus every equation of the hierarchy (4.9) can provide a set of evolutionary, in general generalized, symmetries [65,70-73]. We have described here this procedure in the case of partial differential equations, where it was first introduced. This procedure has then been extended to the case of differential-difference and difference-difference equations $[4,5,12,38,49,60,61,166,175,185,188,222,223]$.

In the case of a differential-difference equation

$$
E\left(n, t, u_{n}(t), u_{n+1}(t), u_{n, t}(t), \ldots\right)=0
$$

the linear operators $L$ and $M$ that characterize it, depend on the shift operators in the discrete variable $n$. The Lax equations (4.7 4.8) are still valid. The recursion operator $\mathcal{L}$ and the Lax pair will depend on the shift operators, instead of $x$ derivatives. This implies that the higher equations of the hierarchy

$$
E_{j}\left(n, t, u_{n}(t), u_{n+1}(t), u_{n, t}(t), \ldots\right)=0
$$

and the higher symmetries will depend on points further away from the point $n$ instead of depending on higher derivatives. The situation is slightly different in the case of difference-difference equations

$$
E\left(n, m, u_{n, m}, u_{n+1, m}, u_{n, m+1}, \ldots\right)=0 .
$$

The Lax pair in this case involves two linear functions $L_{n, m}$ and $M_{n, m}$ of the shift operator in $n$ with coefficients depending on $u_{n, m}$ The the linear equation (4.3) is replaced by

$$
\psi_{n, m+1}=-M_{n, m}(u) \psi_{n, m} .
$$


The Lax equation in the isospectral regime $\left(\lambda_{m+1}=\lambda_{m}\right)$ now reads:

$$
L_{n, m+1} M_{n, m}=M_{n, m} L_{n, m}
$$

and in the nonisospectral case, when $\lambda_{m+1}=f\left(\lambda_{m}\right)$, with $f($.$) an entire function$ of its argument,

$$
L_{n, m+1} M_{n, m}=M_{n, m} f\left(L_{n, m}\right) .
$$

Few results are known on generalized symmetries of difference-difference equations $[127,172,184]$. In [127], using the techniques presented below, we computed the point and generalized symmetries for the discrete time Toda Lattice(2.1). The symmetries are provided by flows commuting with it. We are considering Lie symmetries, so these flows are given by differential difference equations. Indeed the group parameter is a continuous variable. So the symmetries of the discrete time Toda Lattice (2.1) are given by the Toda Lattice hierarchy of nonlinear differential-difference equations (4.20), with the evolution not in the time variable, but in the group parameter.

The Painlevé property for integrable PDEs is not directly applicable. Several approaches have been proposed in the recent years $[2,18,43,44,83-85,96-98$, $193,202-204,228,232]$. The first is the singularity confinement proposed by Grammaticos et. al. [84]. iIn this approach one requires that any singularity of the solution of a discrete system be confined and should not propagate. This criterion is not sufficient, a system can satisfy the singularity confinement, but give rise to numerical chaos [97]. More refined techniques have been proposed later. Among them let us mention that of Conte and Musette [43] in which they propose a natural extension of the Painlevé property to discrete systems, that of Viallet and Hietarinta [18,96-98] based on the study of growth and complexity properties and that of Ablowitz et. al. [2] based on the study of the asymptotic behaviour using the Nevanlinna theory of value distribution and growth of meromorphic functions.

In the following we will construct an infinite class of symmetries for the Toda lattice. We will construct formally all the generalized symmetries and present explicitly the simplest examples. We will show the structure of the algebra of the generalized and point symmetries, using the one-to-one correspondence between the configuration space and the spectral space, where all equations are linear. In the continuous limit the Lie algebra of the point symmetries of the potential KdV (4.70) are recovered, as are the generalized symmetries. Finally, we present the Bäcklund transformations for the Toda lattice and show their relation to the symmetries. For the corresponding results for the Volterra equation, the completely discrete Toda lattice and for the Discrete Nonlinear Schrödinger equation we refer to the literature $[38,89,90,95,127,132]$.

\subsection{The Toda system and its symmetries}

The Toda equation (2.3) can be rewritten in the form of a system

$$
\dot{a}_{n}(t)=a_{n}(t)\left(b_{n}(t)-b_{n+1}(t)\right), \quad \dot{b}_{n}(t)=a_{n-1}(t)-a_{n}(t),
$$


where

$$
b_{n}=\dot{v}_{n}, \quad a_{n}=\mathrm{e}^{\mathrm{v}_{\mathrm{n}}-\mathrm{v}_{\mathrm{n}+1}} .
$$

It can be associated to the discrete Schrödinger spectral problem

$$
\psi(n-1, t ; \lambda)+b_{n} \psi(n, t ; \lambda)+a_{n} \psi(n+1, t ; \lambda)=\lambda \psi(n, t ; \lambda)
$$

The time evolution of the wave function $\psi(n, t ; \lambda)$ is given by

$$
\psi_{t}(n, t ; \lambda)=-a_{n}(t) \psi(n+1, t ; \lambda)
$$

For the point symmetries of the Toda equation see Section 2.3

To the spectral problem (4.18) we can associate a set of nonlinear differential difference equations (the Toda Lattice hierarchy)

$$
\left(\begin{array}{c}
\dot{a}_{n} \\
\dot{b}_{n}
\end{array}\right)=f_{1}(\mathcal{L}, t)\left(\begin{array}{c}
a_{n}\left(b_{n}-b_{n+1}\right) \\
a_{n-1}-a_{n}
\end{array}\right)
$$

where $f_{1}(\mathcal{L}, t)$ is an entire function of its first argument and the recursion operator $\mathcal{L}$, is given by

$$
\mathcal{L}\left(\begin{array}{c}
p_{n} \\
q_{n}
\end{array}\right)=\left(\begin{array}{c}
p_{n} b_{n+1}+a_{n}\left(q_{n}+q_{n+1}\right)+\left(b_{n}-b_{n+1}\right) s_{n} \\
b_{n} q_{n}+p_{n}+s_{n-1}-s_{n}
\end{array}\right) .
$$

The operator (4.21) was firstly obtained by Dodd [49] and by Bruschi, Levi and Ragnisco [26]. In eq. 4.21) $s_{n}$ is a solution of the nonhomogeneous first order equation

$$
s_{n+1}=\frac{a_{n+1}}{a_{n}}\left(s_{n}-p_{n}\right) .
$$

For any equation of the hierarchy (4.20) we can write down an explicit evolution equation for the function $\psi(n, t ; \lambda)[27,28,30]$ such that $\lambda$ does not evolve in time. This is possible if the following boundary conditions

$$
\lim _{|n| \rightarrow \infty} a_{n}-1=\lim _{|n| \rightarrow \infty} b_{n}=\lim _{|n| \rightarrow \infty} s_{n}=0,
$$

are imposed on the fields $a_{n}, b_{n}$ and $s_{n}$. The boundedness of the solutions of eqs. (4.23) was not required in the literature $[25,29,30]$ but it is necessary to get a hierarchy of nonlinear differential-difference equations with well defined evolution of the spectra. We can than associate to eq.4.18) a spectrum $S[u]$ $[27,60,61,175,230]$ defined in the complex plane of the variable $z\left(\lambda=z+z^{-1}\right)$ :

$$
S[u]=\left\{R(z, t), z \in \mathbf{C}_{1} ; z_{j}, c_{j}(t),\left|z_{j}\right|<1, j=1,2, \ldots, N\right\},
$$

where $R(z, t)$ is the reflection coefficient, $\mathbf{C}_{1}$ is the unit circle in the complex $z$ plane, $z_{j}$ are isolated points inside the unit disk and $c_{j}$ are some complex functions of $t$ related to the residues of $R(z, t)$ at the poles $z_{j}$. When $a_{n}, b_{n}$ and $s_{n}$ satisfy the boundary conditions (4.23), the spectral data define the potentials in a unique way. Thus, there is a one-to-one correspondence between the 
evolution of the potentials $\left(a_{n}, b_{n}\right)$ of the discrete Schrödinger spectral problem (4.18), given by eq. (4.20) and that of the reflection coefficient $R(z, t)$, given by

$$
\frac{d R(z, t)}{d t}=\mu f_{1}(\lambda, t) R(z, t), \quad \mu=z^{-1}-z .
$$

In eq. 4.25) and below, $\frac{d}{d t}$ denotes the total derivative with respect to $t$.

The Toda system is obtained from eq. (4.20) by choosing $f_{1}(\lambda, t)=1$ and thus the evolution equation of the reflection coefficient is given by

$$
\frac{d R(z, t)}{d t}=\mu R(z, t) .
$$

The symmetries for the Toda system (4.16), or the Toda equation (2.3) are provided by all flows commuting with the equation itself. Let us introduce the following denumerable set of equations

$$
\left(\begin{array}{c}
a_{n, \epsilon_{k}} \\
b_{n, \epsilon_{k}}
\end{array}\right)=\mathcal{L}^{k}\left(\begin{array}{c}
a_{n}\left(b_{n}-b_{n+1}\right) \\
a_{n-1}-a_{n}
\end{array}\right) .
$$

Here $k$ is any positive integer and $\epsilon_{k}$ is a variable. We can associate to the equation (4.27) an evolution of the reflection coefficient

$$
\frac{d R}{d \epsilon_{k}}=\mu \lambda^{k} R
$$

It can be verified directly that the flows (4.26) and (4.28) commute and hence the same must be true for the corresponding equations, i.e. for the Toda system (4.16) and the equations (4.27). This implies that eqs. (4.27) are symmetries of the Toda system and consequently $\epsilon_{k}$ is a group parameter. From the point of view of the spectral problem (4.18), eqs. (4.27) correspond to isospectral deformations, i.e. we have $\lambda_{\epsilon_{k}}=0$. For any $\epsilon_{k}$, the solution of the Cauchy problem for eq. (4.27), provides a solution of the Toda system (4.16) $\left[a_{n}\left(t, \epsilon_{k}\right), b_{n}\left(t, \epsilon_{k}\right)\right]$ in terms of the initial condition $\left[a_{n}\left(t, \epsilon_{k}=0\right), b_{n}\left(t, \epsilon_{k}=0\right)\right]$. The group transformation corresponding to the group parameter $\epsilon_{k}$ can usually be written explicitly only for the lowest values of $k$. In the case of the generalized symmetries, the group action is obtained in principle by solving the Cauchy problem for the nonlinear characteristic equation starting from a generic solution of the Toda lattice. This often cannot be done. In all cases we can construct just a few classes of explicit group transformations corresponding to very specific solutions of the Toda lattice equation, namely the solitons, the rational solutions and the periodic solutions [230]. In all cases one can use the symmetries (4.27) to do, for example, symmetry reduction and to reduce the equation under consideration to an ordinary differential equation, or possibly a functional one. This is done by looking for fixed pints of the transformation, i.e. putting $a_{n, \epsilon_{k}}=0, b_{n, \epsilon_{k}}=0$,

We can extend the class of symmetries by considering nonisopectral deformations of the spectral problem [4.18] $[65,70,71,132,162]$. Thus for the Toda 
system we obtain

$$
\begin{array}{rc}
\left(\begin{array}{c}
a_{n, \epsilon_{k}} \\
b_{n, \epsilon_{k}}
\end{array}\right)= & \mathcal{L}^{k+1} t\left(\begin{array}{c}
a_{n}\left(b_{n}-b_{n+1}\right) \\
a_{n-1}-a_{n}
\end{array}\right) \\
& +\mathcal{L}^{k}\left(\begin{array}{c}
a_{n}\left[(2 n+3) b_{n+1}-(2 n-1) b_{n}\right] \\
b_{n}^{2}-4+2\left[(n+1) a_{n}-(n-1) a_{n-1}\right]
\end{array}\right) .
\end{array}
$$

In correspondence with eq.(4.29) we have the evolution of the reflection coefficient 4.24, given by

$$
\frac{d R}{d \epsilon_{k}}=\mu \lambda^{k+1} t R, \quad \lambda_{\epsilon_{k}}=\mu^{2} \lambda^{k}
$$

The proof that the eqs. (4.29) are symmetries is done by showing that the flows (4.30) and (4.26) in the space of the reflection coefficients commute.

In addition to the above two hierarchies of symmetries (4.27) and (4.29), we can construct two further cases, which, however, do not satisfy the asymptotic boundary conditions (4.23). They are:

$$
\left(\begin{array}{l}
a_{n, \epsilon} \\
b_{n, \epsilon}
\end{array}\right)=\left(\begin{array}{l}
0 \\
1
\end{array}\right), \quad\left(\begin{array}{c}
a_{n, \epsilon} \\
b_{n, \epsilon}
\end{array}\right)=t\left(\begin{array}{c}
a_{n}\left(b_{n}-b_{n+1}\right) \\
a_{n-1}-a_{n}
\end{array}\right)+\left(\begin{array}{c}
2 a_{n} \\
b_{n}
\end{array}\right) .
$$

As these exceptional symmetries do not satisfy the asymptotic boundary conditions (4.23), we cannot write a corresponding evolution equation for the reflection coefficient (4.24).

Let us now write down the lowest order symmetries for the Toda system (4.16) that one can get from the hierarchies (4.27 4.29). In the case of the Toda lattice (2.3) the symmetries are obtained from those of the Toda system (4.16) by using the transformation (4.17). The symmetries of the Toda lattice and the Toda system, corresponding to the isospectral and nonisospectral flows, will have the same evolution of the reflection coefficient. The transformation (4.17) involves an integration (to obtain $u_{n}$ ). The integration constant must be chosen so as to satisfy the following boundary conditions:

$$
\lim _{|n| \rightarrow \infty} v_{n}=0
$$

In the case of the exceptional symmetries such an integration will provide an additional symmetry.

Taking $k=0,1$, and 2 in eq. (4.27) we obtain the first three isospectral symmetries for the Toda system, namely:

$$
\begin{gathered}
a_{n, \epsilon_{0}}=a_{n}\left(b_{n}-b_{n+1}\right), \\
b_{n, \epsilon_{0}}=a_{n-1}-a_{n}, \\
a_{n, \epsilon_{1}}=a_{n}\left(b_{n}^{2}-b_{n+1}^{2}+a_{n-1}-a_{n+1}\right), \\
b_{n, \epsilon_{1}}=a_{n-1}\left(b_{n}+b_{n-1}\right)-a_{n}\left(b_{n+1}+b_{n}\right),
\end{gathered}
$$




$$
\begin{array}{r}
a_{n, \epsilon_{2}}=a_{n}\left(b_{n}^{3}-b_{n+1}^{3}+a_{n} b_{n}-2 a_{n+1} b_{n+1}+a_{n-1} b_{n-1}+2 a_{n-1} b_{n}\right. \\
\left.-a_{n+1} b_{n+2}-a_{n} b_{n+1}-2 b_{n}+2 b_{n+1}\right), \\
b_{n, \epsilon_{2}}=a_{n-1}\left(b_{n}^{2}+b_{n-1}^{2}+b_{n} b_{n-1}+a_{n-1}+a_{n-2}-2\right) \\
-a_{n}\left(b_{n}^{2}+b_{n+1}^{2}+b_{n} b_{n+1}+a_{n+1}+a_{n}-2\right) .
\end{array}
$$

The lowest nonisospectral symmetry is obtained from eq. (4.29), taking $k=0$. It is:

$$
\begin{array}{r}
a_{n, \nu}=a_{n}\left\{t\left(b_{n}^{2}-b_{n+1}^{2}+a_{n-1}-a_{n+1}\right)+(2 n+3) b_{n+1}-(2 n-1) b_{n}\right\}(4.36) \\
b_{n, \nu}=t\left\{a_{n-1}\left(b_{n}+b_{n-1}\right)-a_{n}\left(b_{n+1}+b_{n}\right)\right\}+b_{n}^{2}-4+2\left[(n+1) a_{n}-(n-1) a_{n-1}\right] .
\end{array}
$$

The nonisospectral symmetries for $k>0$ are nonlocal.

The exceptional symmetries (4.31) are:

$$
\begin{aligned}
& a_{n, \mu_{0}}=0, \quad b_{n, \mu_{0}}=1, \\
& a_{n, \mu_{1}}=2 a_{n}+t \dot{a}_{n}, \quad b_{n, \mu_{1}}=b_{n}+t \dot{b}_{n} .
\end{aligned}
$$

The corresponding symmetries for the Toda lattice are

$$
\begin{gathered}
v_{n, \epsilon_{0}}=\dot{v}_{n} \\
v_{n, \epsilon_{1}}=\dot{v}_{n}^{2}+e^{v_{n-1}-v_{n}}+e^{v_{n}-v_{n+1}}-2 \\
v_{n, \epsilon_{2}}=\dot{v}_{n}^{3}-2 \dot{v}_{n}+e^{v_{n-1}-v_{n}}\left(\dot{v}_{n-1}+2 \dot{v}_{n}\right)+e^{v_{n}-v_{n+1}}\left(\dot{v}_{n+1}+2 \dot{v}_{n}\right) \\
v_{n, \nu}=t\left\{\dot{v}_{n}^{2}+e^{v_{n-1}-v_{n}}+e^{v_{n}-v_{n+1}}-2\right\}-(2 n-1) \dot{v}_{n}+w_{n}(t),
\end{gathered}
$$

where $w_{n}(t)$ is defined by the following compatible system of equations:

$$
w_{n+1}(t)-w_{n}(t)=-2 \dot{v}_{n+1}, \quad \dot{w}_{n}(t)=2\left(e^{v_{n}-v_{n+1}}-1\right) .
$$

Under the assumption (4.32) we can integrate eqs. (4.43) and obtain a formal solution. That is, we can write $w_{n}(t)$ in the form of an infinite sum;

$$
w_{n}(t)=2 \sum_{j=n+1}^{\infty} \dot{v}_{j}+\alpha,
$$

where $\alpha$ is an arbitrary integration constant which can be interpreted as an additional symmetry. The exceptional symmetries read:

$$
\begin{gathered}
v_{n, \mu_{1}}=t \dot{v}_{n}-2 n \\
v_{n, \mu_{0}}=t
\end{gathered}
$$

and the additional one, due to the integration

$$
v_{n, \mu_{-1}}=1 .
$$




\subsubsection{The symmetry algebra for the Toda lattice}

To define the structure of the symmetry algebra for the Toda lattice we need to compute the commutation relations between the symmetries, i.e. between the flows commuting with the equations of the hierarchy. Using the one-to-one correspondence between the integrable equations and the evolution equations for the reflection coefficients, we calculate the commutation relations between the symmetries and thus analyze the structure of the obtained infinite dimensional Lie algebra.

If we define

$$
\mathcal{L}^{k}=\left(\begin{array}{ll}
\mathcal{L}_{11}^{(k)} & \mathcal{L}_{12}^{(k)} \\
\mathcal{L}_{21}^{(k)} & \mathcal{L}_{22}^{(k)}
\end{array}\right),
$$

we can write the generators for the isospectral symmetries as

$$
\begin{aligned}
\hat{X}_{k}^{T}= & \left\{\mathcal{L}_{11}^{(k)}\left[a_{n}\left(b_{n}-b_{n+1}\right)\right]+\mathcal{L}_{12}^{(k)}\left(a_{n-1}-a_{n}\right)\right\} \partial_{a_{n}} \\
+ & \left\{\mathcal{L}_{21}^{(k)}\left[a_{n}\left(b_{n}-b_{n+1}\right)\right]+\mathcal{L}_{22}^{(k)}\left(a_{n-1}-a_{n}\right)\right\} \partial_{b_{n}} .
\end{aligned}
$$

The superscript on the generator $\hat{X}$, is there to indicate that this is the symmetry generator for the Toda system (4.16). We will indicate by the superscript $T L$ the symmetry generators for the Toda Lattice 2.3 . To these generators we can associate symmetry generators in the space of the reflection coefficients. These generators are written as

$$
\hat{\mathcal{X}}_{k}^{T}=\mu \lambda^{k} R \partial_{R}
$$

In agreement with Lie theory, whenever $R$ is an analytic function of $\epsilon$, the corresponding flows are given by solving the equations

$$
\frac{d \tilde{R}}{d \epsilon_{k}}=\mu \lambda^{k} \tilde{R}, \quad \frac{d \tilde{\lambda}}{d \epsilon_{k}}=0, \quad \tilde{R}\left(\epsilon_{k}=0\right)=R, \quad \tilde{\lambda}\left(\epsilon_{k}=0\right)=\lambda .
$$

One can prove that the isospectral symmetry generators (4.49) commute amongst each other

$$
\left[\hat{X}_{k}^{T}, \hat{X}_{m}^{T}\right]=0
$$

by computing the corresponding commutation relation in the space of the reflection coefficients

$$
\left[\hat{\mathcal{X}}_{k}^{T}, \hat{\mathcal{X}}_{m}^{T}\right]=\left[\mu \lambda^{k} R \partial_{R}, \mu \lambda^{m} R \partial_{R}\right]=0 .
$$

So far, the use of the vector fields in the reflection coefficient space has just reexpressed a known result, namely that the commutation of the reflection coefficients is rewritten as eq. 4.53). We now extend the use of vector fields in the space of the spectral data to the case of the nonisospectral symmetries (4.29). 
Using the definition (4.48) we can introduce the generators of the nonisospectral symmetries for the Toda system. The symmetry vector fields are:

$$
\begin{aligned}
\hat{Y}_{k}^{T}=\quad & \left\{t\left[\mathcal{L}_{11}^{(k+1)}\left[a_{n}\left(b_{n}-b_{n+1}\right)\right]+\mathcal{L}_{12}^{(k+1)}\left(a_{n-1}-a_{n}\right)\right]\right. \\
& \quad+\mathcal{L}_{11}^{(k)}\left[a_{n}\left((2 n+3) b_{n+1}-(2 n-1) b_{n}\right)\right] \\
& \left.+\mathcal{L}_{12}^{(k)}\left[b_{n}^{2}-4+2(n+1) a_{n}-2(n-1) a_{n-1}\right]\right\} \partial_{a_{n}} \\
& +\left\{t\left[\mathcal{L}_{21}^{(k+1)}\left[a_{n}\left(b_{n}-b_{n+1}\right)\right]+\mathcal{L}_{22}^{(k+1)}\left(a_{n-1}-a_{n}\right)\right]\right. \\
& \quad+\mathcal{L}_{21}^{(k)}\left[a_{n}\left((2 n+3) b_{n+1}-(2 n-1) b_{n}\right)\right] \\
& \left.+\mathcal{L}_{22}^{(k)}\left[b_{n}^{2}-4+2(n+1) a_{n}-2(n-1) a_{n-1}\right]\right\} \partial_{b_{n}}
\end{aligned}
$$

Taking into account eq. (4.30), we can define the symmetry generators 4.54) in the space of the spectral data, as

$$
\hat{\mathcal{Y}}_{k}^{T}=\mu \lambda^{k+1} t R \partial_{R}+\mu^{2} \lambda^{k} \partial_{\lambda} .
$$

Commuting $\hat{\mathcal{Y}}_{k}^{T}$ with $\hat{\mathcal{Y}}_{m}^{T}$ we have:

$$
\left[\hat{\mathcal{Y}}_{k}^{T}, \hat{\mathcal{Y}}_{m}^{T}\right]=(m-k)\left[\hat{\mathcal{Y}}_{k+m+1}^{T}-4 \hat{\mathcal{Y}}_{k+m-1}^{T}\right] .
$$

From the isomorphism between the spectral space and the space of the solutions, we conclude that the vector fields representing the symmetries of the studied evolution equations, satisfy the same commutation relations

$$
\left[\hat{Y}_{k}^{T}, \hat{Y}_{m}^{T}\right]=(m-k)\left[\hat{Y}_{k+m+1}^{T}-4 \hat{Y}_{k+m-1}^{T}\right],
$$

In a similar manner we can work out the commutation relations between the $\hat{Y}_{k}$ and $\hat{X}_{m}$ symmetry generators. We get:

$$
\left[\hat{\mathcal{X}}_{k}^{T}, \hat{\mathcal{Y}}_{m}^{T}\right]=-(1+k) \hat{\mathcal{X}}_{k+m+1}^{T}+4 k \hat{\mathcal{X}}_{k+m-1}^{T},
$$

and consequently

$$
\left[\hat{X}_{k}^{T}, \hat{Y}_{m}^{T}\right]=-(1+k) \hat{X}_{k+m+1}^{T}+4 k \hat{X}_{k+m-1}^{T} .
$$

Relations like (4.57) and 4.59) can also be checked directly, but the use of vector field in the reflection coefficient space is much more efficient.

Let us now consider the commutation relations involving the exceptional symmetries (4.31). We write them as:

$$
\begin{gathered}
\hat{Z}_{0}^{T}=\partial_{b_{n}} \\
\hat{Z}_{1}^{T}=\left[2 a_{n}+t \dot{a}_{n}\right] \partial_{a_{n}}+\left[b_{n}+t \dot{b}_{n}\right] \partial_{b_{n}} .
\end{gathered}
$$

As mentioned in Section 4.1 these symmetries do not satisfy the asymptotic conditions (4.23). Hence we cannot write down the commutation relations in all generality for all symmetries simultaneously. We calculate explicitly the 
commutation relations involving just $\hat{Z}_{0}^{T}$ and $\hat{Z}_{1}^{T}, \hat{X}_{0}^{T}, \hat{X}_{1}^{T}$ and $\hat{Y}_{0}^{T}$. The non zero commutation relations are:

$$
\begin{aligned}
& {\left[\hat{X}_{0}^{T}, \hat{Z}_{1}^{T}\right]=-\hat{X}_{0}^{T}, \quad\left[\hat{Z}_{0}^{T}, \hat{Z}_{1}^{T}\right]=\hat{Z}_{0}^{T}} \\
& {\left[\hat{Y}_{0}^{T}, \hat{Z}_{0}^{T}\right]=-2 \hat{Z}_{1}^{T}, \quad\left[\hat{Y}_{0}^{T}, \hat{Z}_{1}^{T}\right]=-\hat{Y}_{0}^{T}-8 \hat{Z}_{0}^{T}, \quad\left[\hat{X}_{1}^{T}, \hat{Z}_{0}^{T}\right]=-2 \hat{X}_{0}^{T},} \\
& {\left[\hat{X}_{1}^{T}, \hat{Z}_{1}^{T}\right]=-2 \hat{X}_{1}^{T},\left[\hat{X}_{0}^{T}, \hat{Y}_{0}^{T}\right]=-\hat{X}_{1}^{T},\left[\hat{X}_{1}^{T}, \hat{Y}_{0}^{T}\right]=-2 \hat{X}_{2}^{T}+4 \hat{X}_{0}^{T} .}
\end{aligned}
$$

In the case of the Toda lattice (2.3) we have (see eqs. (4.46] 4.45))

$$
\begin{aligned}
& \hat{Z}_{0}^{T L}=t \partial_{v_{n}} \\
& \hat{Z}_{1}^{T L}=\left[t \dot{v}_{n}-2 n\right] \partial_{v_{n}}
\end{aligned}
$$

and

$$
\hat{Z}_{-1}^{T L}=\partial_{v_{n}}
$$

in correspondence with eq. (4.47). As eqs. (2.3) and (4.16) are just two different representations of the same system, the symmetry generators in the space of the spectral data are the same. Consequently the commutation relations between $\hat{X}_{n}^{T L}$ and $\hat{Y}_{m}^{T L}$ are given by eqs. 4.53 4.57 and 4.59). The symmetries $\hat{X}_{0}^{T L}$, $\hat{X}_{1}^{T L}$ and $\hat{Y}_{0}^{T L}$, according to eqs.4.39), (4.40, (4.42) are given by:

$$
\begin{aligned}
\hat{X}_{0}^{T L}= & \dot{v}_{n} \partial_{v_{n}}, \quad \hat{X}_{1}^{T L}=\left[\dot{v}_{n}^{2}+e^{v_{n-1}-v_{n}}+e^{v_{n}-v_{n+1}}-2\right] \partial_{v_{n}} \\
\hat{Y}_{0}^{T L}= & \left\{t\left[v_{n, t}^{2}+e^{v_{n-1}-v_{n}}+e^{v_{n}-v_{n+1}}-2\right]-(2 n-1) v_{n, t}+w_{n}(t)\right\} \partial_{v_{n}} \\
& w_{n+1}(t)-w_{n}(t)=-2 \dot{v}_{n+1}, \quad \dot{w}_{n}(t)=2\left(e^{v_{n}-v_{n+1}}-1\right)
\end{aligned}
$$

The nonzero commutation relations are:

$$
\begin{aligned}
& {\left[\hat{X}_{0}^{T L}, \hat{Z}_{0}^{T L}\right]=-\hat{Z}_{-1}^{T L}, \quad\left[\hat{X}_{0}^{T L}, \hat{Z}_{1}^{T L}\right]=-\hat{X}_{0}^{T L}} \\
& {\left[\hat{X}_{0}^{T L}, \hat{Y}_{0}^{T L}\right]=-\hat{X}_{1}^{T L}+\omega \hat{Z}_{-1}^{T L},} \\
& {\left[\hat{X}_{1}^{T L}, \hat{Z}_{0}^{T L}\right]=-2 \hat{X}_{0}^{T L}, \quad\left[\hat{X}_{1}^{T L}, \hat{Z}_{1}^{T L}\right]=-2 \hat{X}_{1}^{T L}-4 \hat{Z}_{-1}^{T L},} \\
& {\left[\hat{X}_{1}^{T L}, \hat{Y}_{0}^{T L}\right]=-2 \hat{X}_{2}^{T L}+4 \hat{X}_{0}^{T L}+\sigma \hat{Z}_{-1}^{T L}} \\
& {\left[\hat{Y}_{0}^{T L}, \hat{Z}_{-1}^{T L}\right]=\beta \hat{Z}_{-1}^{T L}, \quad\left[\hat{Y}_{0}^{T L}, \hat{Z}_{0}^{T L}\right]=-2 \hat{Z}_{1}^{T L}+\gamma \hat{Z}_{-1}^{T L},} \\
& {\left[\hat{Y}_{0}^{T L}, \hat{Z}_{1}^{T L}\right]=-\hat{Y}_{0}^{T L}-8 \hat{Z}_{0}^{T L}+\delta \hat{Z}_{-1}^{T L}, \quad\left[\hat{Z}_{0}^{T L}, \hat{Z}_{1}^{T L}\right]=\hat{Z}_{0}^{T L},}
\end{aligned}
$$

where $(\beta, \gamma, \delta, \omega, \sigma)$ are integration constants. The presence of these integration constants indicates that the symmetry algebra of the Toda equation is not completely specified. The constants appear whenever the symmetry $\hat{Y}_{0}^{T L}$ is involved. The ambiguity is related to the ambiguity in the definition of $\hat{Y}_{0}^{T L}$ itself, i.e. in the solution of eq. (4.66) for $w_{n}(t)$. We fix these coefficients by requiring that one obtains the correct continuous limit, i.e. in the asymptotic limit, when $h$ goes to zero, a combination of the generators of the Toda Lattice (2.3) and Toda system (4.16) goes over to the symmetry algebra of the potential Korteweg - de Vries equation (see below).

The commutation relations obtained above determine the structure of the infinite dimensional Lie symmetry algebras. The first symmetry generators are 
given in eqs. (4.49), (4.54), 4.60), (4.61) and the corresponding commutation relations are given by eqs. 4.57), 4.59), 4.62). As one can see, the symmetry operators $\hat{Y}_{k}^{T}$ and $\hat{Z}_{k}^{T}$ are linear in $t$ and the coefficient of $t$ is an isospectral symmetry operator $\hat{X}_{k}^{T}$. Consequently, as the operators $\hat{X}_{k}^{T}$ commute amongst each other, the commutator of $\hat{X}_{m}^{T}$ with any of the $\hat{Y}_{k}^{T}$ or $\hat{Z}_{k}^{T}$ symmetries will not have any explicit time dependence and thus can be written in terms of $\hat{X}_{n}^{T}$ only. Thus the structure of the Lie algebra for the Toda system can be written as:

$$
L=L_{0} \boxplus L_{1}, \quad L_{0}=\left\{\hat{h}, \hat{e}, \hat{f}, \hat{Y}_{1}^{T}, \hat{Y}_{2}^{T}, \cdots\right\}, L_{1}=\left\{\hat{X}_{0}^{T}, \hat{X}_{1}^{T}, \cdots\right\}
$$

where $\left\{\hat{h}=\hat{Z}_{1}^{T}, \hat{e}=\hat{Z}_{0}^{T}, \hat{f}=\hat{Y}_{0}^{T}+4 \hat{Z}_{0}^{T}\right\}$ denotes a sl(2,R) subalgebra with $[\hat{h}, \hat{e}]=\hat{e},[\hat{h}, \hat{f}]=-\hat{f},[\hat{e}, \hat{f}]=2 \hat{h}$. The algebra $L_{0}$ is perfect, i.e. we have $\left[L_{0}, L_{0}\right]=L_{0}$. It is worthwhile to notice that $\hat{Z}_{0}^{T}, \hat{Z}_{1}^{T}$ and $\hat{X}_{0}^{T}$ are point symmetries while all the others are generalized symmetries.

For the Toda lattice equation the point transformations are $\hat{X}_{0}^{T L}, \hat{Z}_{0}^{T L}$ and $\hat{Z}_{1}^{T L}$, as for the Toda system, plus the additional $\hat{Z}_{-1}^{T L}$. Taking into account eqs. (4.63 4.67), the structure of the Lie algebra is the same as that of the Toda system with $L_{0}=\left\{\hat{Z}_{-1}^{T L}, \hat{Z}_{0}^{T L}, \hat{Z}_{1}^{T L}, \hat{Y}_{0}^{T L}, \hat{Y}_{1}^{T L}, \hat{Y}_{2}^{T L}, \cdots\right\}, L_{1}=\left\{\hat{X}_{0}^{T L}, \hat{X}_{1}^{T L}, \hat{X}_{2}^{T L}, \cdots\right\}$.

\subsubsection{Contraction of the symmetry algebras in the continuous limit}

It is well known $[27,30,136,137,230]$, that the Toda equation has the potential Korteweg-de Vries equation as one of its possible continuous limits. In fact, by setting

$$
v_{n}(t)=-\frac{1}{2} h u(x, \tau) \quad x=(n-t) h \quad \tau=-\frac{1}{24} h^{3} t
$$

we can write eq. (2.3) as

$$
\left(u_{\tau}-u_{x x x}-3 u_{x}^{2}\right)_{x}=\mathcal{O}\left(h^{2}\right)
$$

i.e. the once differentiated potential Korteweg-de Vries equation. Let us now rewrite the symmetry generators in the new coordinate system defined by (4.69) and develop them for small $h$ in Taylor series. We have:

$$
\begin{aligned}
\hat{X}_{0}^{T L} & =\left\{-u_{x}(x, \tau) h-\frac{1}{24} u_{\tau}(x, \tau) h^{3}\right\} \partial_{u} \\
\hat{X}_{1}^{T L} & =\left\{-2 u_{x}(x, \tau) h-\frac{1}{3} u_{\tau}(x, \tau) h^{3}+\mathcal{O}\left(h^{5}\right)\right\} \partial_{u} \\
\hat{X}_{2}^{T L} & =\left\{-4 u_{x}(x, \tau) h-\frac{7}{6} u_{\tau}(x, \tau) h^{3}+\mathcal{O}\left(h^{5}\right)\right\} \partial_{u} \\
\hat{Y}_{0}^{T L} & =\left\{2\left[u(x, \tau)+x u_{x}(x, \tau)+3 \tau u_{\tau}(x, \tau)\right]+\mathcal{O}(h)\right\} \partial_{u} \\
\hat{Z}_{-1}^{T L} & =-\frac{2}{h} \partial_{u}, \quad \hat{Z}_{0}^{T L}=\frac{48}{h^{4}} \tau \partial_{u} \\
\hat{Z}_{1}^{T L} & =\left\{-\frac{96}{h^{4}} \tau+\frac{4}{h^{2}}\left[x+6 \tau u_{x}(x, \tau)\right]+\mathcal{O}(1)\right\} \partial_{u} .
\end{aligned}
$$


To obtain eqs. (4.72 4.74) we have used the following evolution for $u$ :

$$
u_{\tau}=u_{x x x}+3 u_{x}^{2} .
$$

The point symmetry generators written in the evolutionary form, for the potential Korteweg-de Vries equation [4.77) read:

$$
\begin{aligned}
\hat{P}_{0} & =u_{\tau} \partial_{u}, \quad \hat{P}_{1}=u_{x} \partial_{u}, \quad \hat{B}=\left[x+6 \tau u_{x}\right] \partial_{u}, \\
\hat{D} & =\left[u+x u_{x}+3 \tau u_{\tau}\right] \partial_{u}, \quad \hat{\Gamma}=\partial_{u},
\end{aligned}
$$

and their commutation table is:

\begin{tabular}{c|ccccc} 
& $\hat{P}_{0}$ & $\hat{P}_{1}$ & $\hat{B}$ & $\hat{D}$ & $\hat{\Gamma}$ \\
\hline$\hat{P}_{0}$ & 0 & 0 & $-6 \hat{P}_{1}$ & $-3 \hat{P}_{0}$ & 0 \\
$\hat{P}_{1}$ & & 0 & $-\hat{\Gamma}$ & $-\hat{P}_{1}$ & 0 \\
$\hat{B}$ & & & 0 & $2 \hat{B}$ & 0 \\
$\hat{D}$ & & & & 0 & $-\hat{\Gamma}$ \\
$\hat{\Gamma}$ & & & & & 0
\end{tabular}

Let us now consider the continuous limit $h \rightarrow 0$ of the symmetry algebra of the Toda equation. We can write the simplest symmetry generators of the Toda equation as a linear combination of the generators (4.71 4.76), so that in the continuous limit they go over to the generators of the point symmetries of the potential Korteweg-de Vries equation (4.78 4.79):

$$
\begin{aligned}
& \tilde{P}_{0}=\frac{4}{h^{3}}\left(2 \hat{X}_{0}^{T L}-\hat{X}_{1}^{T L}\right), \tilde{P}_{1}=-\frac{1}{h} \hat{X}_{0}^{T L}, \quad \tilde{D}=\frac{1}{2} \hat{Y}_{0}^{T L}, \\
& \tilde{B}=\frac{h^{2}}{4}\left(2 \hat{Z}_{0}^{T L}+\hat{Z}_{1}^{T L}\right), \quad \tilde{\Gamma}=-\frac{h}{2} \hat{Z}_{-1}^{T L} .
\end{aligned}
$$

Taking into account the commutation table between the generators $\hat{X}_{0}^{T L}, \hat{X}_{1}^{T L}$, $\hat{Z}_{-1}^{T L}, \hat{Z}_{0}^{T L}, \hat{Z}_{1}^{T L}$ and $\hat{Y}_{0}^{T L}$, given by (4.67) and the continuous limit of $\hat{X}_{2}^{T L}$ given by eq. (4.73), we get:

\begin{tabular}{c|ccccc} 
& $\tilde{P}_{0}$ & $\tilde{P}_{1}$ & $\tilde{B}$ & $\tilde{D}$ & $\tilde{\Gamma}$ \\
\hline$\tilde{P}_{0}$ & 0 & 0 & $-6 \tilde{P}_{1}+\mathcal{O}\left(h^{2}\right)$ & $-3 \tilde{P}_{0}+\mathcal{O}\left(h^{2}\right)$ & 0 \\
$\tilde{P}_{1}$ & & 0 & $-\tilde{\Gamma}+\mathcal{O}\left(h^{2}\right)$ & $-\tilde{P}_{1}+\mathcal{O}\left(h^{2}\right)$ & 0 \\
$\tilde{B}$ & & & 0 & $2 \tilde{B}+\mathcal{O}\left(h^{2}\right)$ & 0 \\
$\tilde{D}$ & & & & 0 & $-\tilde{\Gamma}$ \\
$\tilde{\Gamma}$ & & & & & 0
\end{tabular}

The results contained in table 4.83) are obtained by setting $\beta=-2,2 \gamma+\delta=$ 0 , and $\omega=\sigma=0$. Thus, we have reobtained in the continuous limit, all point symmetries of the potential KdV equation. The limit partially fixes the previously undetermined constants in eq. (4.67). To get all point symmetries of the potential $\mathrm{KdV}$ equation we needed not only the point symmetries $\hat{X}_{0}^{T L}$, 
$\hat{Z}_{0}^{T L}, \hat{Z}_{-1}^{T L}$ and $\hat{Z}_{1}^{T L}$ of the Toda equation, but also the higher symmetries $\hat{X}_{1}^{T L}$, $\hat{Y}_{0}^{T L}$.

This procedure can be viewed as a new application of the concept of Lie algebra contractions. Lie algebra contractions were first introduced by Inönü and Wigner [105] in order to relate the group theoretical foundations of relativistic and nonrelativistic physics. The speed of light $c$ was introduced as a parameter into the commutation relations of the Lorentz group. For $c \rightarrow \infty$ the Lorentz group "contracted" to the Galilei group. Lie algebra contractions thus relate different Lie algebras of the same dimension, but of different isomorphism classes. A systematic study of contractions, relating large families of nonisomorphic Lie algebras of the same dimension, based on Lie algebra grading, was initiated by Moody and Patera [180].

In general Lie algebra and Lie group contractions are extremely useful when describing the mathematical relation between different theories. The contraction parameter can be the Planck constant, when relating quantum systems to classical ones. It can be the curvature $k$ of a space of constant curvature, which for $k \rightarrow 0$ goes to a flat space. The contraction will then relate special functions defined e.g. on spheres, to those defined in a Euclidean space [110].

In our case the contraction parameter is the lattice spacing $h$. Some novel features appear. First of all, we are contracting an infinite dimensional Lie algebra of generalized symmetries, that of the Toda lattice. The contraction leads to an infinite dimensional Lie algebra, not isomorphic to the first one. This "target algebra" is the Lie algebra of point and generalized symmetries of the potential $\mathrm{KdV}$ equation. A particularly interesting feature is that the five dimensional Lie algebra of point symmetries of the potential $\mathrm{KdV}$ is obtained from a subset of point and generalized symmetries of the Toda equation. This 5 dimensional subset is not an algebra (it is not closed under commutations). It does contract into a Lie algebra in the continuous limit.

\subsubsection{Bäcklund transformations for the Toda equation}

In addition to symmetry transformations presented in Section (4.2.1), the Toda system admits Bäcklund transformations [25, 29, 33, 49, 91, 122, 126, 133]. They are discrete transformations (i.e. mappings) that starting from a solution, produce a new solution. Bäcklund transformations commute amongst each other, allowing the definition of a soliton superposition formula that endows the evolution equation with an integrability feature. Using the spectral transform [33] we can write down families of Bäcklund transformations. They are obtained by requiring the existence of two essentially different solutions to the Lax equations (4.2 4.3), $\psi(x, t ; \lambda)$ and $\tilde{\psi}(x, t ; \lambda)$. These two solutions will be associated to two different solutions to the nonlinear partial differential equation (4.1), $u(x, t)$ and $\tilde{u}(x, t)$ and consequently two different Lax pairs $(L(u), M(u))$ and $(L(\tilde{u}), M(\tilde{u}))$. If a transformation exists between $u$ and $\tilde{u}$ than there must exist a transformation between $\psi(x, t ; \lambda)$ and $\tilde{\psi}(x, t ; \lambda)$ and between $(L(u), M(u))$ and $(L(\tilde{u}), M(\tilde{u}))$. This implies that there will exist an operator $D(u, \tilde{u})$, often 
called the Darboux operator which will relate $\tilde{\psi}(x, t ; \lambda)$ and $\psi(x, t ; \lambda)$, i.e.

$$
\tilde{\psi}(x, t ; \lambda)=D(u, \tilde{u}) \psi(x, t ; \lambda) .
$$

Taking into account the Lax equations for $\psi(x, t ; \lambda)$ and $\tilde{\psi}(x, t ; \lambda)$, we get from (4.84) the following operator equations for $D$ :

$$
\begin{aligned}
& \tilde{L}(\tilde{u}) D(u, \tilde{u})=D(u, \tilde{u}) L(u), \\
& D_{t}(u, \tilde{u})=D(u, \tilde{u}) M(u)-M(\tilde{u}) D(u, \tilde{u}) .
\end{aligned}
$$

From eqs. 4.85 4.86 we get a class of Bäcklund transformations, which we will symbolically write as $B_{j}(u(x, t), \tilde{u}(x, t), \ldots)$ characterized by a recursion operator $\Lambda$.

In the discrete case, the technique is basically the same, and in the case of the matrix discrete Schrödinger spectral problem, a generalization of the scalar problem (4.18), the appropriate developments are found in [29]. Specializing to the scalar case, the class of Bäcklund transformations associated to the Toda system (4.16) is given by

$$
\gamma(\Lambda)\left(\begin{array}{c}
\tilde{a}(n)-a(n) \\
\tilde{b}(n)-b(n)
\end{array}\right)=\delta(\Lambda)\left(\begin{array}{c}
\tilde{\Pi}(n) \Pi^{-1}(n+1)(\tilde{b}(n)-b(n+1)) \\
\tilde{\Pi}(n-1) \Pi^{-1}(n)-\tilde{\Pi}(n) \Pi^{-1}(n+1),
\end{array}\right)
$$

where $\gamma(z)$ and $\delta(z)$ are entire functions of their argument and we have denoted

$$
\Pi(n)=\prod_{j=n}^{\infty} a(j), \quad \tilde{\Pi}(n)=\prod_{j=n}^{\infty} \tilde{a}(j) .
$$

Above, $\Lambda$ is the recursion operator

$$
\Lambda\left[\begin{array}{l}
p(n) \\
q(n)
\end{array}\right]=\left[\begin{array}{r}
p(n) b(n+1)+\tilde{a}(n)[q(n)+q(n+1)]+\Sigma(n)[\tilde{b}(n)-b(n+1)] \\
+[a(n)-\tilde{a}(n)] \sum_{j=n}^{\infty} p(j) \\
p(n)+\tilde{b}(n) q(n)-\Sigma(n)+\Sigma(n-1)+[b(n)-\tilde{b}(n)] \sum_{j=n}^{\infty} q(j)
\end{array}\right]
$$

and

$$
\Sigma(n)=\tilde{\Pi}(n)\left[\sum_{j=n}^{\infty} \tilde{\Pi}(j)^{-1} p(j) \Pi(j+1)\right] \Pi^{-1}(n+1) .
$$

In [30] it is proven that whenever $\left(a_{n}, b_{n}\right)$ and $\left(\tilde{a}_{n}, \tilde{b}_{n}\right)$ satisfy the asymptotic conditions (4.23) and the Bäcklund transformations (4.87), the reflection coefficient satisfies the equation

$$
\tilde{R}(\lambda)=\frac{\gamma(\lambda)-\delta(\lambda) z}{\gamma(\lambda)-\delta(\lambda) / z} R(\lambda)
$$


For the Toda lattice (2.3) the one-soliton Bäcklund transformation, when the functions $\gamma(\lambda)$ and $\delta(\lambda)$ are constant, reads:

$\dot{u}(n)-\dot{u}(n+1)=\beta\left\{\mathrm{e}^{u(n+1)-\tilde{u}(n+1)}-\mathrm{e}^{\tilde{u}(n-1)-u(n)}-\mathrm{e}^{u(n)-\tilde{u}(n)}+\mathrm{e}^{\tilde{u}(n)-u(n+1)}\right\}$

Equation (4.92) can be interpreted as a (nonlinear) three point difference equation for $\tilde{u}$, where $u$ is some chosen solution of (2.3).

The formulas 4.87 4.910 provide also much more general transformations, i.e. higher order Bäcklund transformations. If the arbitrary functions $\gamma(\lambda)$ and $\delta(\lambda)$ are polynomials, then we have a finite order Bäcklund transformation that can be interpreted as a composition of a finite number of one-soliton transformations. In more general cases, when $\gamma(\lambda)$ and $\delta(\lambda)$ are entire functions, we face an infinite-order Bäcklund transformations.

In the following section we discuss how Bäcklund transformations are related to continuous symmetry transformations, allowing, albeit formally, an integration of the latter.

\subsubsection{Relation between Bäcklund transformations and higher sym- metries}

A general isospectral higher symmetry of the Toda equation is given by

$$
\left(\begin{array}{c}
a_{n, \epsilon} \\
b_{n, \epsilon}
\end{array}\right)=\phi(\mathcal{L})\left(\begin{array}{c}
a_{n}\left(b_{n}-b_{n+1}\right) \\
a_{n-1}-a_{n}
\end{array}\right)
$$

with the spectum evolution

$$
\frac{d R(\lambda, \epsilon)}{d \epsilon}=\mu \phi(\lambda) R(\lambda, \epsilon)
$$

These equations generalize eq. (4.27), used above. In eqs. 4.93 4.94 the function $\phi$ is an entire function of its argument. Eq. (4.94) can be formally integrated in the spectral parameter $(\lambda)$ space, giving:

$$
R(\lambda, \epsilon)=\exp (\mu \phi(\lambda)) R(\lambda, 0) .
$$

Taking into account the following definitions of $\lambda$ and $\mu$ in terms of $z$

$$
\begin{gathered}
\lambda=\frac{1}{z}+z, \quad \mu=\frac{1}{z}-z, \quad \mu^{2}=\lambda^{2}-4, \\
z=\frac{\lambda-\mu}{2}, \quad \frac{1}{z}=\frac{\lambda+\mu}{2},
\end{gathered}
$$

we can rewrite the general Bäcklund transformation (4.91) as

$$
\tilde{R}(\lambda)=\frac{2-(\lambda-\mu) \beta(\lambda)}{2-(\lambda+\mu) \beta(\lambda)} R(\lambda), \quad \beta(\lambda)=\frac{\delta(\lambda)}{\gamma(\lambda)}
$$

In order to identify a general symmetry transformation with a Bäcklund transformation, and vice versa, we equate $R(\lambda, \epsilon)=\tilde{R}(\lambda)$

$$
\exp (\mu \phi(\lambda))=\frac{2-(\lambda-\mu) \beta(\lambda)}{2-(\lambda+\mu) \beta(\lambda)}
$$


and find that $\phi(\lambda)$ in eq. 4.95) is given by

$$
\phi(\lambda)=\frac{1}{\mu} \ln \frac{2-(\lambda-\mu) \beta(\lambda)}{2-(\lambda+\mu) \beta(\lambda)} .
$$

The rhs of eq. 4.99) must not depend on $\mu$. Relations (4.96) allow us to separate the exponential in (4.98) into two entire components $E_{0}(\lambda)$ and $E_{1}(\lambda)$ satisfying

$$
\exp (\mu \phi(\lambda))=\cosh (\mu \phi(\lambda))+\mu \frac{\sinh (\mu \phi(\lambda))}{\mu}=E_{0}(\lambda)+\mu E_{1}(\lambda) .
$$

Noticing that the rhs of eq. (4.99) is rational in $\mu$, developing $\mu^{2}$ and identifying powers (0th and 1st) of $\mu$, we get a system of two compatible equations

$$
\begin{aligned}
-(2-\lambda \beta) E_{0}+\left(\lambda^{2}-4\right) \beta E_{1} & =-(2-\lambda \beta), \\
-\beta E_{0}+(2-\lambda \beta) E_{1} & =\beta .
\end{aligned}
$$

Eqs. (4.101 4.102) provide us with explicit formulas relating a given general higher symmetry (characterized by $\phi$, and thus $E_{0}, E_{1}$ ) with a general Bäcklund transformation (characterized by $\gamma$ and $\delta$, and thus by $\beta$ ):

$$
\beta(\lambda)=\frac{\delta(\lambda)}{\gamma(\lambda)}=\frac{2 E_{1}}{E_{0}+\lambda E_{1}+1}=\frac{2 \sinh (\mu \phi) / \mu}{\cosh (\mu \phi)+\lambda \sinh (\mu \phi) / \mu+1} .
$$

From this equation we see that whatsoever be the symmetry, we find a Bäcklund transformation, i.e. for an arbitrary function $\phi$ we obtain the two entire functions $\gamma$ and $\delta$. Vice versa, given a general Bäcklund transformation, we can find a corresponding generalized symmetry

$$
E_{0}=-\frac{2\left(\beta^{2}-1\right)+\lambda \beta(2-\lambda \beta)}{2\left(\beta^{2}-\lambda \beta+1\right)}, \quad E_{1}=-\frac{(\lambda \beta-2) \beta}{2\left(\beta^{2}-\lambda \beta+1\right)},
$$

or more explicitly,

$$
\phi(\lambda)=\frac{1}{\mu} \sinh ^{-1}\left[-\mu \frac{(\lambda \beta-2) \beta}{2\left(\beta^{2}-\lambda \beta+1\right)}\right] .
$$

In the case of a one-soliton Bäcklund transformation with $\beta=1$, we have:

$$
E_{0}=-\frac{\lambda}{2}, \quad E_{1}=\frac{1}{2}
$$

and we can write $\phi(\lambda)$ as

$$
\phi(\lambda)=\frac{\sinh ^{-1}\left(\sqrt{\lambda^{2}-4} / 2\right)}{\sqrt{\lambda^{2}-4}} .
$$

In this simple case we can write the symmetry in closed form as an infinite sequence of elementary symmetry transformations:

$$
\phi(\lambda)=\sum_{k=0}^{\infty}\left[\frac{(2 k) ! \pi}{k !(k-1) ! 2^{4 k+2}} \lambda^{2 k}+\frac{1}{2} \frac{k !(k+1) !}{(2 k+2) !} \lambda^{2 k+1}\right] .
$$


In this way, the existence of a one-soliton transformation implies the existence of an infinite-order generalized symmetry.

Let us consider the time shift symmetry given by $\phi(\lambda)=1$. Then eq. 4.100) implies that $E_{0}=\cosh \mu$ and $E_{1}=\sinh \mu / \mu$. According to (4.103) the corresponding higher Bäcklund transformation is

$$
\begin{aligned}
\delta(\lambda) & =2 \sinh \mu / \mu \\
\gamma(\lambda) & =\cosh \mu+\lambda \sinh \mu / \mu+1
\end{aligned}
$$

This Bäcklund transformation, corresponding to the point symmetry studied, is of infinite order.

\section{Point Symmetries Transforming Solutions and Lattices}

\subsection{Lie Point Symmetries of Ordinary Difference Schemes}

\subsubsection{Ordinary Difference Schemes}

In this section we take a complementary view to the previous ones. We shall restrict ourselves to continuous point symmetries only. On the other hand we shall consider flexible difference schemes, rather than just difference equations on fixed lattices. For brevity and simplicity of exposition, we limit ourselves to ordinary difference schemes $(\mathrm{O} \Delta \mathrm{S})$. For partial difference schemes, involving more than one independent variable, see Ref. [55,143].

An $\mathrm{O} \Delta \mathrm{S}$ involves two objects, a difference equation and a lattice. We shall specify an $\mathrm{O} \Delta \mathrm{S}$ by a system of two equations, both involving two continuous variables $x$ and $u(x)$, evaluated at a discrete set of points $\left\{x_{n}\right\}$.

Thus, a difference scheme of order $K$ will have the form

$$
\begin{gathered}
E_{a}\left(\left\{x_{k}\right\}_{k=n+M}^{n+N},\left\{u_{k}\right\}_{k=n+M}^{n+N}\right)=0, a=1,2 \\
K=N-M+1, \quad n, M, N \in \mathbb{Z}, \quad N \geq M, \quad u_{k} \equiv u\left(x_{k}\right) .
\end{gathered}
$$

At this stage we are not imposing any boundary conditions, so the reference point $x_{n}$ can be arbitrarily shifted to the left, or to the right. The order $K$ of the system is the number of points involved in the scheme (5.1) and it is assumed to be finite. We also assume that if the values of $x_{k}$ and $u_{k}$ are specified at $(N-M)$ neighbouring point, we can calculate their values at the point to the right, or to the left of the given set, using equations (5.1) i.e., the Jacobians satisfy

$$
\frac{\partial\left(E_{1}, E_{2}\right)}{\partial\left(x_{n+N}, u_{n+N}\right)} \neq 0, \quad \frac{\partial\left(E_{1}, E_{2}\right)}{\partial\left(x_{n+M}, u_{n+M}\right)} \neq 0 .
$$

A continuous limit, when the spacings between all neighbouring points go to zero, if it exists, will take one of the equations (5.1) into a differential equation of order $K^{\prime} \leq K$, the other into an identity (like $0=0$ ). 
When taking the continuous limit it is convenient to introduce different quantities, namely differences between neighbouring points and discrete derivatives like

$$
\begin{aligned}
h_{+}\left(x_{n}\right) & =x_{n+1}-x_{n}, \quad h_{-}\left(x_{n}\right)=x_{n}-x_{n-1}, \\
u_{x} & =\frac{u_{n+1}-u_{n}}{x_{n+1}-x_{n}}, \quad u_{\underline{x}}=\frac{u_{n}-u_{n-1}}{x_{n}-x_{n-1}}, \\
u_{x \underline{x}} & =2 \frac{u_{, x}-u_{, \underline{x}}}{x_{n+1}-x_{n-1}}, \ldots
\end{aligned}
$$

In the continuous limit, we have

$$
h_{+} \rightarrow 0, \quad h_{-} \rightarrow 0, \quad u_{, x} \rightarrow u^{\prime}, \quad u_{\underline{x}} \rightarrow u^{\prime}, \quad u_{x \underline{x}} \rightarrow u^{\prime \prime} .
$$

As a clarifying example of the meaning of the difference scheme (5.1), let us consider a three-point scheme that will approximate a second-order linear difference equation:

$$
\begin{aligned}
& E_{1}=\frac{u_{n+1}-2 u_{n}+u_{n-1}}{\left(x_{n+1}-x_{n}\right)^{2}}-u_{n}=0, \\
& E_{2}=x_{n+1}-2 x_{n}+x_{n-1}=0 .
\end{aligned}
$$

The solution of eq. $E_{2}=0$, determines a uniform lattice

$$
x_{n}=h n+x_{0} .
$$

The scale $h$ and the origin $x_{0}$ in eq. (5.6) are not fixed by eq. (5.5), instead they appear as integration constants, i.e., they are a priori arbitrary. Once they are chosen, eq. (5.4) reduces to a linear difference equation with constant coefficients, since we have $x_{n+1}-x_{n}=h$. Thus, a solution of eq. (5.4) will have the form

$$
u_{n}=\lambda^{x_{n}}
$$

Substituting (5.7) into (5.4) we obtain the general solution of the difference scheme (5.4, (5.5),

$$
\begin{aligned}
u\left(x_{n}\right) & =c_{1} \lambda_{1}^{x_{n}}+c_{2} \lambda_{2}^{x_{n}}, \quad x_{n}=h n+x_{0}, \\
\lambda_{1,2} & =\left(\frac{2+h^{2} \pm h \sqrt{4+h^{2}}}{2}\right)^{1 / 2} .
\end{aligned}
$$

The solution (5.8) of system (5.4) - (5.5) depends on 4 arbitrary constants $c_{1}, c_{2}$, $h$ and $x_{0}$.

Now let us consider a general three-point scheme of the form

$$
E_{a}\left(x_{n-1}, x_{n}, x_{n+1}, u_{n-1}, u_{n}, u_{n+1}\right)=0, \quad a=1,2,
$$

satisfying (5.2) with $N=1, M=-1$ (possibly after an up or down shifting). The two conditions on the Jacobians (5.2) are sufficient to allow us to calculate 
$\left(x_{n+1}, u_{n+1}\right)$ if $\left(x_{n-1}, u_{n-1}, x_{n}, u_{n}\right)$ are known. Similarly, $\left(x_{n-1}, u_{n-1}\right)$ can be calculated if $\left(x_{n}, u_{n}, x_{n+1}, u_{n+1}\right)$ are known. The general solution of the scheme (5.9) will hence depend on 4 arbitrary constants and will have the form

$$
\begin{aligned}
& u_{n}=f\left(x_{n}, c_{1}, c_{2}, c_{3}, c_{4}\right) \\
& x_{n}=\phi\left(n, c_{1}, c_{2}, c_{3}, c_{4}\right) .
\end{aligned}
$$

A more standard approach to difference equations would be to consider a fixed equally spaced lattice, e.g., with spacing $h=1$. We can then identify the continuous variable $x$, sampled at discrete points $x_{n}$, with the discrete variable $n$,

$$
x_{n}=n .
$$

Instead of a difference scheme we then have a difference equation, lke the one we considered in Section 5

$$
E\left(\left\{u_{k}\right\}_{k=n+M}^{n+N}\right)=0,
$$

involving $K=N-M+1$ points. Its general solution has the form

$$
u_{n}=f\left(n, c_{1}, c_{2}, \ldots c_{N-M}\right) \text {, }
$$

i.e., it depends on $N-M$ constants.

Below, when studying point symmetries of discrete equations we will see the advantage of considering difference systems like system (5.1). A treasury of information on difference equations and their solutions can be found in the classical book by Milne-Thompson [179].

\subsubsection{Point Symmetries of Ordinary Difference Schemes}

In this section we shall follow rather closely the article [141]. We shall define the symmetry group of an ordinary difference scheme in the same manner as for ODEs: it is, a group of continuous local point transformations of the form (1.2) taking solutions of the $\mathrm{O} \Delta \mathrm{S}$ (5.1) into solutions of the same scheme. The transformations considered being continuous, we will adopt an infinitesimal approach, as in eq. (1.3). We drop the labels $i$ and $\alpha$, since we are considering the case of one independent and one dependent variable only.

As in the case of differential equations, our basic tool will be vector fields of the form (1.4). In the case of $\mathrm{O} \Delta \mathrm{S}$ they will have the form

$$
X=\xi(x, u) \partial_{x}+\phi(x, u) \partial_{u}
$$

with

$$
x \equiv x_{n}, \quad u \equiv u_{n}=u\left(x_{n}\right) .
$$

Because we are considering point transformations, $\xi$ and $\phi$ in (5.15) depend on $x$ and $u$ at one point only. 
The prolongation of the vector field $X$ is different from that of the case of ODEs. Instead of prolonging to derivatives, we prolong to all points of the lattice figuring in scheme (5.1). Thus we set

$$
\operatorname{pr} X=\sum_{k=n+M}^{n+N} \xi\left(x_{k}, u_{k}\right) \partial_{x_{k}}+\sum_{k=n+M}^{n+N} \phi\left(x_{k}, u_{k}\right) \partial_{u_{k}} .
$$

In these terms the requirement that the transformed function $\tilde{u}(\tilde{x})$ should satisfy the same $\mathrm{O} \Delta \mathrm{S}$ as the original $u(x)$ is expressed by the requirement

$$
\left.\operatorname{pr} X E_{a}\right|_{E_{1}=E_{2}=0}=0, \quad a=1,2 .
$$

Since we must respect both the difference equation and the lattice, we have two conditions (5.17) from which to determine $\xi(x, u)$ and $\phi(x, u)$. Since each of these functions depends on a single point $(x, u)$ and the prolongation (5.16) introduces $N-M+1$ points in space $X \times U$, equation (5.17) will imply a system of determining equations for $\xi$ and $\phi$. Moreover, in general this will be an overdetermined system of linear functional equations that we transform into an overdetermined system of linear differential equations $[9,10]$.

Let us first of all check that the prolongations (5.16) have the continuous limit. We consider the first prolongation in the discrete case

$$
\begin{aligned}
p r^{1} X=\quad & \xi(x, u) \partial_{x}+\phi(x, u) \partial_{u}+\xi\left(x_{+}, u_{+}\right) \partial_{x_{+}}+\phi\left(x_{+}, u_{+}\right) \partial_{u_{+}} \\
& x_{+} \equiv x_{n+1}, \quad u_{+} \equiv u_{n+1}
\end{aligned}
$$

and apply it to a function of the variables $x, u, h_{+}, u_{x}$ (5.3) . The continuous limit is recovered by taking $h \rightarrow 0$ and putting

$$
\begin{aligned}
u_{+} & =u\left(x_{+}\right)=u(x)+h u^{\prime}(x)+\ldots, \\
\xi\left(x_{+}, u_{+}\right) & =\xi(x, u)+\xi_{, x} h+\xi_{, u} u^{\prime} h+\ldots \\
\phi\left(x_{+}, u_{+}\right) & =\phi(x, u)+\phi_{, x} h+\phi_{, u} u^{\prime} h+\ldots
\end{aligned}
$$

where $u^{\prime}$ is the (continuous) derivative of $u(x)$. We have

$$
\begin{array}{r}
p r_{D}^{1} X F\left(x, u, h, u_{x}\right)=\left\{\xi \partial_{x}+\phi \partial_{u}+\left[\xi_{, x}+\xi_{, u} u^{\prime}\right] h \partial_{h}+\right. \\
\left.+\left[-u^{\prime}\left(\xi_{, x}+\xi_{, u} u^{\prime}\right)+\phi_{, x}+\phi_{, u} u^{\prime}\right] \partial_{u_{x}}\right\} F .
\end{array}
$$

In the limit when $h \rightarrow 0$ we obtain

$$
\begin{array}{r}
\lim _{h \rightarrow 0} p r_{D}^{1} X=\xi(x, u) \partial_{x}+\phi(x, u) \partial_{u}+\phi^{x}\left(x, u, u^{\prime}\right) \partial_{u^{\prime}} \\
\phi^{x}=\phi_{, x}+\left(\phi_{, u}-\xi_{, x}\right) u^{\prime}-\xi_{, u} u^{\prime 2}
\end{array}
$$

which is the correct expression for the first prolongation in the continuous case [194]. The proof that the $n$-th prolongation (5.16) has the correct continuous limit can be performed by induction. 


\subsection{Examples}

1. Power nonlinearity on a uniform lattice.

Let us consider a difference scheme that is a discretization of the ODE

$$
u^{\prime \prime}-u^{N}=0, \quad N \not \equiv 0,1 .
$$

For $N \not \equiv-3$, eq. (5.22) is invariant under a two-dimensional Lie group whose Lie algebra is given by

$$
X_{1}=\partial_{x}, \quad X_{2}=(N-1) x \partial_{x}-2 u \partial_{u}
$$

(translations and dilations). For $N=-3$ the symmetry algebra is threedimensional, isomorphic to $\operatorname{sl}(2, \mathbb{R})$, i.e., it contains a third element in addition to (5.23). A convenient basis for the symmetry algebra of the equation

$$
u^{\prime \prime}-u^{-3}=0
$$

is

$$
X_{1}=\partial_{x}, \quad X_{2}=2 x \partial_{x}+u \partial_{u}, \quad X_{3}=x\left(x \partial_{x}+u \partial_{u}\right) .
$$

A very natural $\mathrm{O} \Delta \mathrm{S}$ that has (5.22) as its continuous limit is

$$
\begin{aligned}
& E_{1}=\frac{u_{n+1}-2 u_{n}+u_{n-1}}{\left(x_{n+1}-x_{n}\right)^{2}}-u_{n}^{N}=0 \quad N \not \equiv 0,1 \\
& E_{2}=x_{n+1}-2 x_{n}+x_{n-1}=0 .
\end{aligned}
$$

Let us now apply the symmetry algorithm described in Section 5.1 .2 to system (5.26)-(5.27). To illustrate the method, we shall present all calculations in detail.

First, we choose two variables that will be substituted in eq. (5.17), once the prolonged vector field (5.16) is applied to system (5.26) (5.27), namely

$$
\begin{aligned}
& x_{n+1}=2 x_{n}-x_{n-1} \\
& u_{n+1}=\left(x_{n}-x_{n-1}\right)^{2} u_{n}^{N}+2 u_{n}-u_{n-1}
\end{aligned}
$$

We apply pr $X$ of (5.16) to eq. (5.27) and obtain

$$
\xi\left(x_{n+1}, u_{n+1}\right)-2 \xi\left(x_{n}, u_{n}\right)+\xi\left(x_{n-1}, u_{n-1}\right)=0
$$

where, $x_{n}, u_{n} x_{n-1}, u_{n-1}$ are independent, but $x_{n+1}, u_{n+1}$ are expressed in terms of these quantities, as in eq. (5.28). Taking this into acccount, we differentiate (5.29) first with respect to $u_{n-1}$, then with respect to $u_{n}$. We obtain successively

$$
\begin{aligned}
-\xi_{, u_{n+1}}\left(x_{n+1}, u_{n+1}\right)+\xi_{, u_{n-1}}\left(x_{n-1}, u_{n-1}\right) & =0 \\
\left(N\left(x_{n}-x_{n-1}\right)^{2} u_{n}^{N-1}+2\right) \xi_{, u_{n+1} u_{n+1}}\left(x_{n+1}, u_{n+1}\right) & =0 .
\end{aligned}
$$

Eq. (5.31) is the desired one-term equation. It implies that

$$
\xi(x, u)=a(x) u+b(x) .
$$


Substituting (5.32) into (5.30) we obtain

$$
-a\left(2 x_{n}-x_{n-1}\right)+a\left(x_{n-1}\right)=0 .
$$

Differentiating with respect to $x_{n}$, we obtain $a=a_{0}=$ const. Finally, we substitute (5.32) with $a=a_{0}$ into (5.29) and obtain

$$
a=a_{0}=0, \quad b\left(2 x_{n}-x_{n-1}\right)-2 b\left(x_{n}\right)+b\left(x_{n-1}\right)=0,
$$

and hence

$$
\xi=b=b_{1} x+b_{0},
$$

where $b_{0}$ and $b_{1}$ are constants. To obtain the function $\phi\left(x_{n}, u_{n}\right)$, we apply $\operatorname{pr} X$ to eq. (5.26) and obtain

$$
\begin{aligned}
\phi\left(x_{n+1}, u_{n+1}\right)-2 \phi\left(x_{n}, u_{n}\right)+\phi\left(x_{n-1}, u_{n-1}\right) \\
-\left(x_{n}-x_{n-1}\right)^{2}\left(N \phi\left(x_{n}, u_{n}\right) u_{n}^{N-1}+2 b_{1} u_{n}^{N}\right)=0 .(5.3
\end{aligned}
$$

Differentiating successively with respect to $u_{n-1}$ and $u_{n}$ (taking (5.28) into account), we obtain

$$
\begin{aligned}
-\phi_{, u_{n+1}}\left(x_{n+1}, u_{n+1}\right)+\phi_{, u_{n-1}}\left(x_{n-1}, u_{n-1}\right) & =0 \\
\left(N\left(x_{n}-x_{n-1}\right)^{2} u_{n}^{N}+2\right) \phi_{, u_{n+1} u_{n+1}} & =0
\end{aligned}
$$

and hence

$$
\phi=\phi_{1} u+\phi_{0}(x), \quad \phi_{1}=\text { const } .
$$

Eq. (5.36) now reduces to

$$
\begin{gathered}
\phi_{0}\left(2 x_{n}-x_{n-1}\right)-2 \phi_{0}\left(x_{n}\right)+\phi_{0}\left(x_{n-1}\right) \\
-\left(x_{n}-x_{n-1}\right)^{2}\left((N-1) \phi_{1}+2 b_{1}\right) u_{n}^{N} \\
-N\left(x_{n}-x_{n-1}\right)^{2} \phi_{0} u_{n}^{N-1}=0 .
\end{gathered}
$$

We have $N \not \equiv 0,1$ and hence (5.40) implies that

$$
\phi_{0}=0, \quad(N-1) \phi_{1}+2 b_{1}=0 .
$$

We have thus proven that the symmetry algebra of the $\mathrm{O} \Delta \mathrm{S}(5.26)-(5.27)$ is the same as that of the ODE (5.22), namely the algebra (5.23).

We observe that the value $N=-3$ is not distinguished here and that system (5.26) - (5.27) is not invariant under $S L(2, \mathbb{R})$ for $N=-3$. Actually, a difference scheme invariant under $S L(2, \mathbb{R})$ does exist and it will have eq. (5.24) as its continuous limit. It will not however have the form (5.26)-(5.27), and the lattice will not be uniform $[56,57]$. The corresponding $\mathrm{SL}(2, \mathrm{R})$ invariant scheme is presented below in Section 5.3 .3

Had we taken a two-point lattice, $x_{n+1}-x_{n}=h$ with $h$ fixed, instead of $E_{2}=0$ as in (5.27), we would only have obtained translational invariance for the equation (5.26) and lost the dilational invariance represented by $X_{2}$ of eq. (5.23). 
2. An $O \Delta S$ involving an arbitrary function on a uniform lattice

We consider

$$
\begin{aligned}
& E_{1}=\frac{u_{n+1}-2 u_{n}+u_{n-1}}{\left(x_{n+1}-x_{n}\right)^{2}}-f\left(\frac{u_{n}-u_{n-1}}{x_{n}-x_{n-1}}\right)=0, \\
& E_{2}=x_{n+1}-2 x_{n}+x_{n-1}=0,
\end{aligned}
$$

where $f(z)$ is some sufficiently smooth function satisfying

$$
f^{\prime \prime}(z) \not \equiv 0 .
$$

The continuous limit of eq. (5.42) and (5.43) is

$$
u^{\prime \prime}-f\left(u^{\prime}\right)=0,
$$

and it is invariant under a two-dimensional group with Lie algebra,

$$
X_{1}=\partial_{x}, \quad X_{2}=\partial_{u},
$$

for any function $f\left(u^{\prime}\right)$. For certain functions $f$ the symmetry group is threedimensional, where the additional basis element of the Lie algebra is

$$
X_{3}=(a x+b u) \partial_{x}+(c x+d u) \partial_{u}
$$

Now let us consider the discrete system (5.42) - Before applying $\operatorname{pr} X$ to this system we choose two variables to substitute in eq. (5.17), namely

$$
\begin{aligned}
& x_{n+1}=2 x_{n}-x_{n-1} \\
& u_{n+1}=2 u_{n}-u_{n-1}+\left(x_{n}-x_{n-1}\right)^{2} f\left(\frac{u_{n}-u_{n-1}}{x_{n}-x_{n-1}}\right) .
\end{aligned}
$$

Applying pr $X$ to eq. (5.43) and performing the same kind of passages necessary to solve the determining equation as we did for eq. (5.28) we get

$$
\xi=\alpha x+\beta,
$$

with $\alpha=$ const, $\beta=$ const. Now let us apply pr $X$ to eq. (5.42). We get the following equation for $\phi$

$$
\begin{aligned}
\phi\left(x+h, u+h z+h^{2} f(z)\right)-2 \phi(x, u)+\phi(x-h, u-h z) \\
=2 \alpha h^{2} f(z)+h^{2} f^{\prime}(z)\left(\frac{\phi(x, u)-\phi(x-h, u-h z)}{h}-\alpha z\right) .
\end{aligned}
$$

where we have defined

$$
z=\frac{u_{n}-u_{n-1}}{x_{n}-x_{n-1}}, \quad h=x_{n+1}-x_{n} .
$$

In general, eq. (5.50) is quite difficult to solve and for most functions $f(z)$ it has no further solutions. 
However, if we make the choice

$$
f(z)=e^{-z}
$$

we find that eq. (5.50) is solved by putting

$$
\alpha=1, \quad \phi=x+u .
$$

Finally, we find that the system

$$
\frac{u_{n+1}-2 u_{n}+u_{n-1}}{\left(x_{n+1}-x_{n}\right)^{2}}=e^{\frac{u_{n}-u_{n-1}}{x_{n}-x_{n-1}}}, \quad x_{n+1}-2 x_{n}+x_{n-1}=0
$$

is left invariant by a three-dimensional transformation group, generated by the solvable Lie algebra with basis

$$
X_{1}=\partial_{x} ; \quad X_{2}=\partial_{u} ; \quad X_{3}=x \partial_{x}+(x+u) \partial_{u} .
$$

For further examples see the original article [141] and the lectures [238].

\subsection{Symmetry Preserving Discretization of Ordinary Dif- ferential Equations}

\subsubsection{General Comments}

In Section 5.1 we assumed that an $\mathrm{O} \Delta \mathrm{S}$ (5.1) is given and we showed how to determine its symmetries.

Here we will discuss a different problem, namely the construction of $\mathrm{O} \Delta \mathrm{S}$ with a priori given symmetry groups. More specifically, we start from a given ODE

$$
E(x, y, \dot{y}, \ddot{y}, \ldots)=0
$$

and its symmetry algebra, realized by vector fields of the form (5.15). We now wish to construct an $\mathrm{O} \Delta \mathrm{S}$ (5.1), approximating the ODE (5.56) and having the same symmetry algebra (and the same symmetry group).

This can be done systematically, once the order of the ODE (5.56) is fixed. In general, the motivation for such a study is multifold. In physical applications the symmetry may actually be more important than the equation itself. A discrete scheme with the correct symmetries has a good chance of describing the physics correctly. This is specially true if the underlying phenomena really are discrete and the differential equations come from a continuum approximation. Furthermore, the existence of point symmetries for differential and difference equations makes it possible to obtain explicit analytical solutions. Finally, a discretization respecting point symmetries should provide improved numerical methods.

Let us first outline the general method of discretization. If the ODE (5.56) is of order $N$ we need a $\mathrm{O} \Delta \mathrm{S}$ involving at least $N+1$ points, i.e. $N+1$ pairs

$$
\left\{x_{i}, y_{i} ; i=1, \ldots, N+1\right\} .
$$

The procedure is as follows 
1. Take the Lie algebra $\mathfrak{g}$ of the symmetry group of the ODE (5.56) and prolong the (known) vector fields $\left\{X_{1}, \ldots, X_{n}\right\}$ to all $N+1$ points (5.57), as in eq. (5.16).

2. Find a basis for all invariants of the (prolonged) Lie algebra in the space (5.57) of independent and dependent variables. Such a basis will consist of $K$ functionally independent invariants

$$
I_{a}=I_{a}\left(x_{1}, \ldots, x_{N+1}, y_{1}, \ldots, y_{N+1}\right), \quad 1 \leq a \leq K .
$$

They are determined by solving the differential equations

$$
\operatorname{pr} X_{i} I_{a}\left(x_{1}, \ldots, x_{N+1}, y_{1}, \ldots, y_{N+1}\right)=0, \quad i=1, \ldots, n .
$$

The actual number $K$ satisfies

$$
K=2 N+2-\left(\operatorname{dim} \mathfrak{g}-\operatorname{dim}_{0}\right)
$$

where $\mathfrak{g}_{0}$ is the Lie algebra of the group $\mathfrak{G}_{0} \subset \mathfrak{G}$, stabilizing the $N+1$ points (5.57).

We need at least two independent invariants of the form (5.58) to write an invariant difference scheme.

3. If the number of invariants is not sufficient, we can make use of invariant manifolds. To find them, we first write out the matrix of coefficients of the prolonged vector fields $\left\{X_{1}, \ldots, X_{n}\right\}$ :

$$
M=\left(\begin{array}{cccccccc}
\xi_{11} & \xi_{12} & \ldots & \xi_{1 N+1} & \phi_{11} & \phi_{12} & \ldots & \phi_{1 N+1} \\
\vdots & \vdots & & \vdots & \vdots & \vdots & & \vdots \\
\xi_{n 1} & \xi_{n 2} & \ldots & \xi_{n N+1} & \phi_{n 1} & \phi_{n 2} & \ldots & \phi_{n N+1}
\end{array}\right)
$$

and determine the manifolds on which the $\operatorname{rank}$ of $M, \operatorname{Rank}(M)$, satisfies

$$
\operatorname{Rank}(M)<\min (n, 2 N+2),
$$

i.e. is less than maximal. The invariant manifolds are then obtained by requiring that eq. (5.59) be satisfied on the manifold satisfying eq. (5.62).

\subsubsection{Symmetries of Second Order ODEs}

Let us now restrict to the case of a second order ODE

$$
\ddot{u}=F(x, u, \dot{u}) \text {. }
$$

Sophus Lie gave a symmetry classification of second order ODE's (over the field of complex numbers $\mathbb{C}$ ) $[163,164]$. A similar classification over $\mathbb{R}$ is much more recent $[173,174]$.

The main classification results can be summed up as follows. 
1. The dimension $n=$ dimg of the symmetry algebra of eq. (5.63) can be $\operatorname{dimg}=0,1,2,3$ or 8 .

2. If we have $\operatorname{dimg}=1$ we can decrease the order of eq. (5.63) by one. If the dimension is $\operatorname{dim} \mathfrak{g} \geq 2$ we can integrate by quadratures.

3. If we have $\operatorname{dimg}=8$, then the symmetry algebra is $\operatorname{sl}(3, \mathbb{C})$, or $\operatorname{sl}(3, \mathbb{R})$, respectively. The equation can be transformed into $\ddot{y}=0$ by a point transformation.

Further symmetry results are due to E. Noether [191] and Bessel-Hagen [19]. Every ODE (5.63) can be interpreted as an Euler-Lagrange equation for some Lagrangian density

$$
\mathcal{L}=\mathcal{L}(x, u, \dot{u})
$$

The equation is

$$
\frac{\partial \mathcal{L}}{\partial u}-D\left(\frac{\partial \mathcal{L}}{\partial \dot{u}}\right)=0, \quad D=\frac{\partial}{\partial x}+\dot{u} \frac{\partial}{\partial u}+\ddot{u} \frac{\partial}{\partial \dot{u}}+\ldots
$$

An infinitesimal divergence symmetry, or a Lagrangian symmetry is a vector field $X$ (5.15) satisfying

$$
\operatorname{pr} X(\mathcal{L})+\mathcal{L} D(\xi)=D(V), \quad V=V(x, u),
$$

where $V$ is some function of $x$ and $u$. A symmetry of the Lagrangian $\mathcal{L}$ is always a symmetry of the Euler-Lagrange equation (5.65), however equation (5.65) may have additional, non-Lagrangian symmetries.

A relevant symmetry result is that if we have $\operatorname{dim} \mathfrak{g}=1$, or $\operatorname{dim} \mathfrak{g}=2$ for eq. (5.63), then there always exists a Lagrangian having the same symmetry. For $\operatorname{dim} \mathcal{L}=3$, at least a two-dimensional subalgebra of the Lagrangian symmetries exists. For $\operatorname{dimg}=8$ a four-dimensional solvable subalgebra of Lagrangian symmetries exists.

\subsubsection{Symmetries of the Three-point Difference Schemes}

A symmetry classification of three-point difference schemes was performed quite recently $[56,57]$. It is similar to Lie's classification of second order ODE's and goes over into this classification in the continuous limit. We shall now review the main results of the classification following the method outlined in Section 5.3 .1 above.

Sophus Lie [165] gave a classification of all finite dimensional Lie algebras that can be realized by vector fields of the form (5.15). This was done over the field $\mathbb{C}$ and thus amounts to a classification of finite dimensional subalgebras of $\operatorname{diff}(2, \mathbb{C})$, the Lie algebra of the group of diffeomorphisms of the complex plane $\mathbb{C}^{2}$. A similar classification of finite dimensional subalgebras of $\operatorname{diff}(2, \mathbb{R})$ exists [81], but we restrict ourselves to the simpler complex case.

We use the following notation for 3 neighbouring points on the lattice:

$$
x_{-}=x_{n-1}, x=x_{n}, x_{+}=x_{n+1}, u_{-}=u_{n-1}, u=u_{n}, u_{+}=u_{n+1} .
$$

Let us now proceed by dimension of the symmetry algebras. 
$\operatorname{dim} \mathfrak{g}=1 \quad$ A single vector field can always be rectified into the form

$$
A_{1,1}: \quad X_{1}=\frac{\partial}{\partial u}
$$

The invariant ODE is

$$
\ddot{u}=F(x, \dot{u}) .
$$

Putting $\dot{u}=y$ we obtain a first order ODE.

The difference invariants of $X_{1}$ are

$$
x, h_{+}=x_{+}-x, h_{-}=x-x_{-}, \eta_{+}=u_{+}-u, \eta_{-}=u-u_{-} .
$$

Using these invariants and the notation (5.3), we can write a difference scheme

$$
y_{x \underline{x}}=F\left(x, \frac{u_{x}+u_{\underline{x}}}{2}, h_{-}\right), \quad h_{+}=h_{-} G\left(x, \frac{u_{x}+u_{\underline{x}}}{2}, h_{-}\right) .
$$

This scheme goes into eq. (5.69) if we require that the otherwise arbitrary functions $F$ and $G$ satisfy

$$
\lim _{h_{-} \rightarrow 0} F\left(x, \frac{u_{x}+u_{\underline{x}}}{2}, h_{-}\right)=F(x, \dot{u}), \quad \lim _{h_{-} \rightarrow 0} G\left(x, \frac{u_{x}+u_{\underline{x}}}{2}, h_{-}\right)<\infty .
$$

$\operatorname{dimg}=2$ Precisely four equivalence classes of two-dimensional subalgebras of $\operatorname{diff}(2, \mathbb{C})$ exist. Let us consider them separately.

$A_{2,1}$

$$
X_{1}=\partial_{x}, \quad X_{2}=\partial_{u}
$$

The algebra $A_{2,1}$ is Abelian, the elements $X_{1}$ and $X_{2}$ are linearly nonconnected (linearly independent in any point $(x, y)$ ). The invariant ODE is

$$
\ddot{u}=F(\dot{u}),
$$

and can be immediately integrated.

An invariant difference scheme is given by any two relations between the invariants $h_{+}, h_{-}, \eta_{+}, \eta_{-}$of eq. (5.70), for instance

$$
u_{x \underline{x}}=F\left(\frac{u_{x}+u_{\underline{x}}}{2}, h_{-}\right), h_{+}=h_{-} G\left(\frac{u_{x}+u_{\underline{x}}}{2}, h_{-}\right),
$$

with conditions (5.72) imposed on the functions $F$ and $G$.

$A_{2,2}$

$$
X_{1}=\partial_{u}, \quad X_{2}=x \partial_{x}+u \partial_{u}
$$

This Lie algebra in non-Abelian, the two elements are linearly nonconnected. The invariant ODE is

$$
\ddot{u}=\frac{1}{x} F(\dot{u}) .
$$


A basis for the difference invariance is

$$
\left\{x u_{x \underline{x}}, u_{x}+u_{\underline{x}}, \frac{h_{+}}{h_{-}}, \frac{h_{-}}{x}\right\}
$$

so a possible invariant difference scheme is

$$
u_{x \underline{x}}=\frac{1}{x} F\left(\frac{u_{x}+u_{\underline{x}}}{2}, \frac{h_{-}}{x}\right), h_{+}=h_{-} G\left(\frac{u_{x}+u_{\underline{x}}}{2}, \frac{h_{-}}{x}\right) .
$$

$A_{2,3}$

$$
X_{1}=\partial_{u}, \quad X_{2}=x \partial_{u}
$$

The algebra is Abelian, the elements $X_{1}$ and $X_{2}$ are linearly connected. The invariant ODE is

$$
\ddot{u}=F(x) .
$$

This equation is linear and hence has an eight dimensional symmetry algebra (of which $A_{2,3}$ is just a subalgebra).

The difference invariants are

$$
\left\{u_{x \bar{x}}, x, h_{+}, h_{-}\right\}
$$

so the invariant difference scheme will also be linear (at least in the dependent variable $u$ ).

$A_{2,4}$

$$
X_{1}=\partial_{u}, \quad X_{2}=u \partial_{u}
$$

The algebra is non-Abelian and isomorphic to $A_{2,2}$, but with linearly connected elements. The invariant ODE is again linear,

$$
\ddot{u}=F(x) \dot{u},
$$

as is the invariant difference scheme. Eq. (5.84) is invariant under the group $S L(3, \mathbb{C})$. Difference invariants are

$$
\left\{\xi=2 \frac{u_{x \underline{x}}}{u_{x}+u_{\underline{x}}}, \quad x, h_{+}, h_{-}\right\}
$$

and a possible invariant $\mathrm{O} \Delta \mathrm{S}$ is

$$
2 \frac{u_{x \underline{x}}}{u_{x}+u_{\underline{x}}}=F\left(x, h_{-}\right), \quad G\left(x, h_{+}, h_{-}\right)=0 .
$$

$\operatorname{dimg}=3$ We now turn to difference schemes invariant under three-dimensional symmetry groups. We will restrict ourselves to the case when the corresponding ODE is nonlinear. Hence we will omit all algebras that contain $A_{2,3}$ or $A_{2,4}$ subalgebras (they were considered in [56]). 
$A_{3,1}$

$$
X_{1}=\partial_{x}, \quad X_{2}=\partial_{u}, \quad X_{3}=x \partial_{x}+k u \partial_{u}, k \neq 0, \frac{1}{2}, 1,2
$$

The invariant ODE is

$$
\ddot{u}=\dot{u}^{\frac{k-2}{k-1}} .
$$

For $k=1$ there is no invariant second order equation; for $k=2$ the equation is linear, for $k=\frac{1}{2}$ it is trasformable into a linear equation and has a larger symmetry group.

Difference invariants are

$$
I_{1}=\frac{h_{+}}{h_{-}}, \quad I_{2}=u_{x} h_{+}^{1-k}, \quad I_{3}=u_{\underline{x}} h_{-}^{1-k} .
$$

A simple invariant difference scheme is

$u_{\underline{x}}=\left(\frac{u_{x}+u_{\underline{x}}}{2}\right)\left[\frac{k-2}{k-1} f\left(\frac{u_{x}+u_{\underline{x}}}{2} h_{-}^{1-k}\right), \quad h_{+}=h_{-} g\left(\frac{u_{x}+u_{\underline{x}}}{2} h_{-}^{1-k}\right)\right.$.

We shall see below that other invariant schemes may be more convenient.

$A_{3,2}$

$$
X_{1}=\partial_{x}, \quad X_{2}=\partial_{u}, \quad X_{3}=x \partial_{x}+(x+u) \partial_{u},
$$

The invariant ODE is

$$
\ddot{u}=e^{-\dot{u}} .
$$

Difference invariants in this case are

$$
I_{1}=\frac{h_{+}}{h_{-}}, \quad I_{2}=h_{+} e^{-u_{x}}, \quad I_{3}=h_{-} e^{-u_{x}} .
$$

A possible invariant scheme is

$$
u_{x \underline{x}}=e^{-\frac{u_{x}+u_{\underline{x}}}{2}} f\left(\sqrt{h_{-} h_{+}} e^{-\frac{u_{x}+u_{\underline{x}}}{2}}\right), \quad h_{+}=h_{-} g\left(\sqrt{h_{-} h_{+}} e^{-\frac{u_{x}+u_{\underline{x}}}{2}}\right) .
$$

No further solvable three-dimensional subalgebras of $\operatorname{diff}(2, \mathbb{C})$ exist (though there is another family for $\operatorname{diff}(2, \mathbb{R})[56]$ ).

Two inequivalent realizations of $s l(2, \mathbb{C})$ exist. Let us consider them separately.

$A_{3,3}$

$$
X_{1}=\partial_{x}, \quad X_{2}=2 x \partial_{x}+u \partial_{u}, \quad X_{3}=x^{2} \partial_{x}+x u \partial_{u},
$$

The corresponding invariant ODE is

$$
\ddot{u}=u^{-3},
$$


and its general solution is

$$
u^{2}=A\left(x-x_{0}\right)^{2}+\frac{1}{A}, \quad A \neq 0 .
$$

A convenient set of difference invariants is

$$
\begin{aligned}
& I_{1}=\frac{h_{+}}{u u_{+}}, \quad I_{2}=\frac{h_{-}}{h_{+}+h_{-}} \frac{u_{+}}{u}, \\
& I_{3}=\frac{h_{+}}{h_{+}+h_{-}} \frac{u_{-}}{u}, \quad I_{4}=\frac{h_{-}}{u u_{-}} .
\end{aligned}
$$

Any three of these are independent; the four satisfy the identity

$$
I_{1} I_{2}=I_{3} I_{4}
$$

An invariant difference scheme can be written as

$$
2\left(I_{2}+I_{3}-1\right)=I_{1}^{2} I_{2} \frac{I_{2}+I_{3}}{I_{3}} f\left(I_{1} I_{2}\right), \quad I_{1}+I_{4}=4 I_{1} I_{2} g\left(I_{1} I_{2}\right),
$$

i.e.

$$
\begin{aligned}
& u_{x \bar{x}}=\frac{1}{h_{+}+h_{-}} \frac{1}{u^{2}}\left(\frac{h_{+}}{u_{+}}+\frac{h_{-}}{u_{-}}\right) f\left(\frac{1}{u^{2}} \frac{h_{+} h_{-}}{h_{+}+h_{-}}\right), \\
& \frac{h_{+}}{u_{+}}+\frac{h_{-}}{u_{-}}=\frac{4}{u} \frac{h_{+} h_{-}}{h_{+}+h_{-}} g\left(\frac{1}{u^{2}} \frac{h_{+} h_{-}}{h_{+}+h_{-}}\right) .
\end{aligned}
$$

For $f=g=1$ this scheme approximates the ODE (5.96).

$A_{3,4}$

$$
X_{1}=\partial_{u}, \quad X_{2}=x \partial_{x}+u \partial_{u}, \quad X_{3}=x^{2} \partial_{x}+\left(-x^{2}+u^{2}\right) \partial_{u}
$$

This algebra is again $s l(2, \mathbb{C})$ and can be transformed into

$$
Y_{1}=\partial_{x}+\partial_{u}, \quad Y_{2}=x \partial_{x}+u \partial_{u}, \quad Y_{3}=x^{2} \partial_{x}+u^{2} \partial_{u}
$$

The realization (5.103) (and hence also (5.102)) is imprimitive; (5.91) is primitive. Hence $A_{3.4}$ and $A_{3.3}$ are not equivalent. The invariant ODE for the algebra (5.102) is

$$
x \ddot{u}=C\left(1+\dot{u}^{2}\right)^{\frac{3}{2}}+\dot{u}\left(1+\dot{u}^{2}\right),
$$

where $C$ is a constant. The general integral of eq. (5.104) can be written as

$$
\begin{aligned}
\left(x-x_{0}\right)^{2}+\left(u-u_{0}\right)^{2} & =\left(\frac{x_{0}}{C}\right)^{2}, \quad C \neq 0, \\
x^{2}+\left(u-u_{0}\right)^{2} & =x_{0}^{2}, C=0,
\end{aligned}
$$


where $x_{0}$ and $u_{0}$ are integration constants.

The difference invariants corresponding to the algebra (5.103) are

$$
\begin{array}{r}
I_{1}=\frac{x_{+}-x}{x_{+} x}\left(1+u_{x}^{2}\right), \quad I_{2}=\frac{x-x_{-}}{x_{-} x}\left(1+u_{\underline{x}}^{2}\right), \\
I_{3}=-\frac{\left(x_{+}-x\right)\left(x-x_{-}\right)}{2 x x_{+} x_{-}}\left\{\left(h_{+} u_{x}^{2}+x_{+}+x\right) u_{\underline{x}}+\right. \\
\left.+\left(h_{-} u_{\underline{x}}^{2}-x_{-}-x\right) u_{x}\right\} .
\end{array}
$$

An invariant scheme representing the ODE (5.104) can be written as

$$
I_{3}=C\left(\frac{I_{1}+I_{2}}{2}\right)^{\frac{3}{2}}, \quad I_{1}=I_{2},
$$

(this is not the most general such scheme).

\subsubsection{Lagrangian formalism and solutions of three-point $\mathrm{O} \Delta \mathrm{S}$}

In Section 5.3 .2 we presented a Lagrangian formalism for the integration of second order ODE's. Let us now adapt it to $\mathrm{O} \Delta \mathrm{S}[57]$.

The Lagrangian density (5.64) will now be a two-point function

$$
\mathcal{L}=\mathcal{L}\left(x, u, x_{+}, u_{+}\right) .
$$

Instead of the Euler-Lagrange equation (5.65) we have two quasiextremal equations $[51,54,57]$ corresponding to "discrete variational derivatives" of $\mathcal{L}$ with respect to $x$ and $y$ independently

$$
\begin{aligned}
& \frac{\delta \mathcal{L}}{\delta x}=h_{+} \frac{\partial \mathcal{L}}{\partial x}+h_{-} \frac{\partial \mathcal{L}^{-}}{\partial x}+\mathcal{L}^{-}-\mathcal{L}=0 \\
& \frac{\delta \mathcal{L}}{\delta u}=h_{+} \frac{\partial \mathcal{L}}{\partial u}+h_{-} \frac{\partial \mathcal{L}^{-}}{\partial u}=0
\end{aligned}
$$

where $\mathcal{L}^{-}$is obtained by downshifting $\mathcal{L}$ (replacing $n$ by $n-1$ everywhere). In the continuous limit both quasiextremal equations reduce to the same Euler-Lagrange equation. Thus, the two quasiextremal equations together can be viewed as an $\mathrm{O} \Delta \mathrm{S}$, where e.g. the difference between them defines the lattice.

The Lagrangian density (5.109) will be divergence invariant under the transformation generated by vector field $X$, if it satisfies

$$
\operatorname{pr} X(\mathcal{L})+\mathcal{L} D_{+}(\xi)=D_{+}(V)
$$

for some function $V(x, u)$ where $D_{+}(f)$ is the discrete total derivative

$$
D_{+} f(x, u)=\frac{f(x+h, u(x+h))-f(x, u)}{h}
$$


Each infinitesimal Lagrangian divergence symmetry operator $X$ will provide one first integral of the quasiextremal equation

$$
h_{-} \phi \frac{\partial \mathcal{L}^{-}}{\partial u}+h_{-} \xi \frac{\partial \mathcal{L}^{-}}{\partial x}+\xi \mathcal{L}^{-}-V=K
$$

[57]. These first integrals will have the form

$$
f_{a}\left(x, x_{+}, u, u_{+}\right)=K_{a}, \quad a=1, \ldots
$$

Thus, if we have two first integrals, we are left with a two point $\mathrm{O} \Delta \mathrm{S}$ to solve. If we have three first integrals, then the quasiextremal equations reduce to a single two point difference equation, e.g. involving just $x_{n}$ and $x_{n+1}$. This can often be solved explicitly [179].

This procedure has been systematically applied to three point $\mathrm{O} \Delta \mathrm{S}$ in the original article [57]. For brevity we will just consider some examples here.

Let us first consider a two-dimensional Abelian Lie algebra and the corresponding invariant second order ODE:

$$
X_{1}=\partial_{x}, X_{2}=\partial_{u}, \quad \ddot{u}=F(\dot{u}) .
$$

This equation is the Euler-Lagrange equation for the Lagrangian

$$
\mathcal{L}=u+G(\dot{u}), \ddot{G}=\frac{1}{F},
$$

and both symmetries are Lagrangian ones

$$
\operatorname{pr} X_{1} \mathcal{L}+\mathcal{L} D\left(\xi_{1}\right)=0, \operatorname{pr} X_{2} \mathcal{L}+\mathcal{L} D\left(\xi_{2}\right)=1=D(x) .
$$

The corresponding two first integrals are

$$
J_{1}=u+G(\dot{u})-\dot{u} \dot{G}(\dot{u}), \quad J_{2}=\dot{G}(\dot{u})-x
$$

Introducing $H$ as the inverse function of $\dot{G}$ we have

$$
\dot{y}=H\left(J_{2}+x\right), H\left(J_{2}+x\right)=[\dot{G}]^{-1}\left(J_{2}+x\right) .
$$

Substituting into the first equation in eq. (5.118), we obtain the general solution of (5.115) as

$$
y(x)=J_{1}-G\left[H\left(J_{2}+x\right)\right]+\left(J_{2}+x\right) H\left(J_{2}+x\right) .
$$

Now let us consider the discrete case. We introduce the discrete analogue of (5.116) as

$$
\mathcal{L}=\frac{u+u_{+}}{2}+G\left(u_{x}\right)
$$


for some smooth function $G$. Equations (5.117) hold (with $D$ interpreted as the discrete total derivative $\left.D_{+}\right)$. The two quasiextremal equations are

$$
\begin{array}{r}
\frac{x_{+}-x_{-}}{2}-\dot{G}\left(u_{x}\right)+\dot{G}\left(u_{\underline{x}}\right)=0, \\
u_{x} \dot{G}\left(u_{x}\right)-u_{\underline{x}} \dot{G}\left(u_{\underline{x}}\right)-G\left(u_{x}\right)+G\left(u_{\underline{x}}\right)-\frac{u_{+}-u_{-}}{2}=0
\end{array}
$$

The two first integrals obtained using Noether's theorem in this case can be written as

$$
\begin{aligned}
\dot{G}\left(u_{x}\right)-\frac{x+x_{+}}{2} & =B \\
-u_{x} \dot{G}\left(u_{x}\right)+G\left(u_{x}\right)+u+\frac{1}{2}\left(x_{+}-x\right) u_{x} & =A .
\end{aligned}
$$

In principle, these two integrals can be solved to obtain

$$
u_{x}=H\left[B+\frac{1}{2}\left(x_{+}+x\right)\right], \quad u=\Phi\left(A, B, x, x_{+}\right),
$$

where $H[z]=[\dot{G}]^{-1}(z)$ and $\Phi$ is obtained by solving eq. (5.124), once $u_{x}=H$ is substituted into this equation. A three point difference equation for $x_{n+2}, x_{n+1}$ and $x_{n}$, not involving $u$ is obtained from the consistency condition $u_{x}=\frac{u_{n+1}-u_{n}}{x_{n+1}-x_{n}}$. In general this equation is difficult to solve. We shall follow a different procedure which is less general, but works well when the considered $\mathrm{O} \Delta \mathrm{S}$ has a three dimensional solvable symmetry algebra with $\left\{\partial_{x}, \partial_{u}\right\}$ as a subalgebra. We add a third equation to the system (5.123, 5.124, namely

$$
\frac{x_{+}-x}{x-x_{-}}=1+\epsilon
$$

The general solution of eq. (5.126) is

$$
x_{n}=\left(x_{0}+B\right)(1+\epsilon)^{n}-B
$$

where $x_{0}$ and $B$ are integration constants. We will identify $B$ with the constant in eq. (5.123), but leave $\epsilon$ as an arbitrary constant. Eq. (5.127) defines an exponential lattice (for $\epsilon \neq 0$ ). Using (5.127) together with (5.123) and (5.124), we find

$$
\begin{array}{r}
u_{x}=H\left[\left(x_{n}+B\right)\left(1+\frac{\epsilon}{2}\right)\right], \quad H[z]=[\dot{G}]^{-1}(z)(5 \\
u_{n}=A+\left(x_{n}+B\right) H\left[\left(x_{n}+B\right)\left(1+\frac{\epsilon}{2}\right)\right]-G\left[H\left(x_{n}+B\right)\left(1+\frac{\epsilon}{2}\right)\right](5
\end{array}
$$

There is no guarantee that equation (5.128) and (5.129) are compatible. However, let us consider the two special cases with three-dimensional solvable symmetry algebras, namely algebras $A_{3,1}$ and $A_{3,2}$ of Section 5.3.3 
Algebra $A_{3,1}$. We choose $G\left(u_{x}\right)$ to be

$$
G\left(u_{x}\right)=\frac{(k-1)^{2}}{k} u_{x}^{\frac{k}{k-1}}, \quad k \neq 0,1
$$

From eq. (5.128) and (5.129) we obtain

$$
\begin{array}{r}
u_{x}=\left(\frac{1}{k-1}\right)^{k-1} x_{n}^{k-1}\left(1+\frac{\epsilon}{2}\right)^{k-1} \\
u_{n}=\frac{1}{k}\left(\frac{1}{k-1}\right)^{k-1}\left(x_{n}+B\right)^{k}\left(1+\frac{\epsilon}{2}\right)^{k-1}\left[1+(1-k) \frac{\epsilon}{2}\right]
\end{array}
$$

The consistency condition (for $u_{x}$ to be the discrete derivative of $u_{n}$ ) provides us with a transcedental equation for $\epsilon$ :

$$
\left[(1+\epsilon)^{k}-1\right]\left[1+(1-k) \frac{\epsilon}{2}\right]=k \epsilon .
$$

In the continuous limit we take $\epsilon \rightarrow 0$ and $u_{n}$ given by eq. (5.132) goes to the general solution of the ODE (5.86). In eq. (5.133) terms of order $\epsilon^{0}, \epsilon$, and $\epsilon^{2}$ cancel. The solution $u_{n}$ coincides with the continuous limit up to terms of order $\epsilon^{2}$.

We mention that in the special case $k=-1$ all three symmetries of the $\mathrm{O} \Delta \mathrm{S}$ are Lagrangian ones and in this case eq. (5.133) is identically satisfied for any $\epsilon$.

Algebra $A_{3,2}$. We choose $G\left(u_{x}\right)$ to be

$$
G\left(u_{x}\right)=e^{u_{x}}
$$

and obtain

$$
\begin{aligned}
u_{x}= & \ln \left(x_{n}+B\right)\left(1+\frac{\epsilon}{2}\right), \\
u_{n}= & \left(x_{n}+B\right) \ln \left(x_{n}+B\right)+A+ \\
& +\left(x_{n}+B\right)\left[\ln \left(1+\frac{\epsilon}{2}\right)-\left(1+\frac{\epsilon}{2}\right)\right] .
\end{aligned}
$$

The expressions (5.135) and (5.136) are consistent if $\epsilon$ satisfies

$$
\epsilon\left(1+\frac{\epsilon}{2}\right)-(1+\epsilon) \ln (1+\epsilon)=0
$$

Again (5.136) coincides with its continuous limit up to terms of order $\epsilon^{2}$ and in (5.137) terms of order $\epsilon^{0}, \epsilon^{1}$ and $\epsilon^{2}$ cancel.

For the $\operatorname{sl}(3, \mathbb{R})$ algebra $A_{3,3}$ all three symmetry operators $X_{1}, X_{2}$ and $X_{3}$ correspond to Lagrangian symmetries. The corresponding $\mathrm{O} \Delta \mathrm{S}$ is integrated in [57]. 


\section{Conclusions and Open Problems}

Let us compare the symmetry approach for difference equations with that for differential ones. In both cases one is interested in transformations taking solutions into solutions and in both cases, choices have to be made. Lie's choice of point symmetries of differential equations is so natural, that it is often forgotten that it is also just an Ansatz : the vector fields should depend on the independent and dependent variables only. Generally speaking, this Ansatz has turned out to be the most useful one. Contact symmetries have much fewer applications than point ones. Generalized symmetries are mainly of use for identifying integrable nonlinear partial differential equations.

In this review we have stressed the fact that for difference equations this choice of pure point transformations must be modified. Without significant modifications it remains mainly fruitful for differential-difference equations rather than for purely difference ones (see Section 2).

Which modifications are needed for difference equations depends on the application that we have in mind. For differential equations there are two main types of applications in physics. In the first, the equations are already known and group theory is used to solve them. In the second, the symmetries of the physical problem are known and are used in building up the theoretical model, i.e. the symmetries precede the equations. These two aspects are also present in the case of difference equations, but there are new features. First of all, the physical processes that are being described may be discrete and the lattices involved may be real physical objects. If we are considering linear theories, like quantum mechanics, or quantum field theory on a lattice, then the generalized point symmetries of Section 3 are extremely promising. A mathematical tool, umbral calculus, is ready to be used, both to solve equations and also to formulate models. For nonlinear theories on given fixed lattices the most appropriate symmetry approach involves generalized symmetries, as reviewed in Section 4. Their main application is as in the continuous case: to identify integrable systems on lattices. Moreover it can be used to get new interesting solutions. An interesting feature is that some point symmetries of differential equations, in particular dilations, appear as generalized symmetries for difference equations.

The second type of application of difference equations in physics is more practical and in a certain sense, less fundamental. We have in mind the situation when the physical processes are really continuous and are described by differential equations. These are then discretized in order to solve them. The lattices used are then our choice and they can be chosen in a symmetry adapted way. Moreover, as shown in Section [5] the difference equation and lattice are both part of a "difference scheme" and the actual lattice is part of the solution of this scheme. We can then restrict ourselves to point transformations, but they act simultaneously on the solutions and on the lattice.

In an attempt to keep this review reasonably short, we have left out many interesting and important topics. Among them we have not included a complete discussion of partial difference equations on transforming symmetry-adapted lattices $[55,58,143]$ and the use of Lie point symmetries to get conditions for the 
linearizability of difference equations [31,86,120,170,201,214,216]. Also left out is the vast area of numerical methods of solving differential equations, making use of their symmetry properties [80] or the treatment of asymptotic symmetries for difference equations [76]. A very active area of research, not covered in the present review, is the use of higher symmetries to identify integrable lattice equations $[12,13,158-161,223,240]$.

Since we are reviewing a relatively new area of research, many open questions remain. Thus, for purely difference equations on fixed lattices, the role of discrete symmetries has not been fully explored. Basically, what is needed, specially for partial difference equations, is to apply results from crystallography, to characterize discrete or finite transformations taking a lattice into itself. For differential-difference equations with three independent variables, one or two of them discrete, a classification of equations with Kac-Moody-Virasoro symmetries should help to identify new integrable lattice equations. Applications to genuine physical systems would be of great interest. The umbral approach of Section 3 has so far been applied in a rather formal manner. The question of the convergence of formal power series solutions must be addressed. The main question is whether one can develop a complete convergent quantum theory on a lattice. The generalized symmetries of Section 4 are an integral part of the theory of integrability on a lattice. There the greatest challenge lies in the field of applications, i.e in applying the techniques of integrability to the real world of discrete phenomena. Finally, the greatest challenge in the direction of symmetry adapted discretizations is to establish whether they provide improved numerical methods, in particular for partial differential equations, or higher order ordinary ones.

\section{Acknowledgments}

The research of P.W. was partly supported by a research grant from NSERC of Canada. Both authors benefitted from the NATO collaborative grant PST.CLG. 978431. D.L. was partially supported by PRIN Project "SINTESI-2000" of the Italian Minister for Education and Scientific Research and from the Projects Sistemi dinamici nonlineari discreti: simmetrie ed integrabilitá and Simmetria e riduzione di equazioni differenziali di interesse fisico-matematico of GNFMINdAM.

\section{References}

[1] Ablowitz M J and Clarkson P A 1991 Solitons, nonlinear evolution equations and inverse scattering (CUP, Cambridge).

[2] Ablowitz M J, Halburd R and Herbst B 2000 On the extension of the Painlevé property to difference equations, Nonlinearity 13 889-905. 
[3] Ablowitz M J, Kaup D J, Newell A C and Segur H 1974 The inverse scattering transform - Fourier analysis for nonlinear problems Stud. Appl. Math. 53 249-318

[4] Ablowitz M J and Ladik J F 1975 Nonlinear differential-difference equations J. Math. Phys. 16 598-603

[5] Ablowitz M J and Ladik J F 1976 A nonlinear difference scheme and inverse scattering Stud. Appl. Math. 55 213-229

[6] Ablowitz M J, Ramani A and Segur H 1978 Nonlinear Evolution Equations and Ordinary Differential Equations of Painlevé Type Lett. Nuovo Cim. 23 333-338.

[7] Ablowitz M J, Ramani A and Segur H 1980 A Connection between Nonlinear Evolution Equations and Ordinary Differential Equations of P-type. I and II J. Math. Phys. 21 715-721 and 1006-1015.

[8] Ablowitz M J and Segur H 1981 Solitons and the inverse scattering transform (SIAM, Philadelphia).

[9] Aczel J 1966 Lectures on Functional Equations and their Applications (AP, New York).

[10] Aczel J (Editor) 1984 Functional Equations: History, Applications and Theory (Reidel, Amsterdam).

[11] Adler V E 2001 Discrete equations on planar graphs J. Phys. A: Math. Gen. 34 10453-10460.

[12] Adler V E, Shabat A B and Yamilov R 2000 Symmetry Approach to the Integrability Problem Theor. Math. Phys. 125 1603-1661.

[13] Adler V E, Svinolupov S I and Yamilov R I 1999 Multi-component Volterra and Toda type integrable equations. Phys. Lett. A $25424-36$.

[14] Ames W F 1972 Nonlinear Partial Differential Equations in Engineering (AP, New York).

[15] Anderson R L and Ibragimov N H 1979 Lie-Bäcklund Transformations in Applications (SIAM, Philadelphia).

[16] Bă̌kov V A, Gazizov R K and Ibragimov N Kh 1988 Approximate symmetries. (Russian) Mat. Sb. (N.S.) 136(178) 435-450, 590; translation in Math. USSR-Sb. 64 (1989) 427-441.

[17] Bakirova M, Dorodnitsyn V and Kozlov R 1997 Invariant difference schemes for heat transfer equations with a source, J. Phys. A: Math. Gen. 30 8139-8155. 
[18] Bellon M P and Viallet C M 1999 Algebraic entropy Comm. Math. Phys. 204 425-437.

[19] Bessel-Hagen E 1921 Uber die Erhaltungssatze der Elektrodynamik Math. Ann. 84, 258-276.

[20] Bluman G W 1990 Simplifying the form of Lie groups admitted by a given differential equation J. Math. Anal. Appl. 145 52-62.

[21] Bluman G and Anco S 2002 Symmetry and Integration Methods for Differential Equations (Springer \& Verlag, New York).

[22] Bluman G W and Cole J D 1969 The general similarity solutions of the heat equation J. Math. Mech. 18 1025-1042.

[23] Bluman G W and Kumei S 1989 Symmetries of Differential Equations (Springer-Verlag, New York).

[24] Boiti M, Bruschi M, Pempinelli F and Prinari B 2003 A discrete Schrödinger spectral problem and associated evolution equations J. Phys. A: Math. Gen. 36 139-149.

[25] Bruschi M, Levi D and Ragnisco O 1980 Extension of the Zakharov-Shabat generalized inverse method to solve differential-difference and differencedifference equations Nuovo Cimento 58A 56-66.

[26] Bruschi M, Levi D and Ragnisco O 1980 Toda lattice and generalised Wronskian technique J. Phys. A: Math. Gen. 13 2531-2533.

[27] Bruschi M, Manakov S V, Ragnisco O and Levi D 1980 The nonabelian Toda lattice-Discrete analogue of the matrix Schrödinger equation $J$. Math. Phys. 21 2749-2753.

[28] Bruschi M and Ragnisco O 1981 Nonlinear differential-difference matrix equations with $n$-dependent coefficients Lett. Nuovo Cimento $31492-496$.

[29] Bruschi M and Ragnisco O 1981 Nonlinear differential-difference equations, associated Bäcklund transformations and Lax technique J. Phys. A: Math. Gen. 14 1075-1081.

[30] Bruschi M, Ragnisco O and Levi D 1981 Evolution equations associated with the discrete analog of the matrix Schrödinger spectral problem solvable by the inverse spectral transform J. Math. Phys. 22 2463-2471.

[31] Byrnes G B, Sahadevan R, and Quispel G R W 1995 Factorizable Lie symmetries and the linearization of difference equations, Nonlinearity 8 $443-459$.

[32] Calogero F 1975 Bäcklund transformations and functional relation for solutions of nonlinear partial differential equations solvable via the inversescattering method Lett. Nuovo Cimento 14 537-543. 
[33] Calogero F and Degasperis A 1982 Spectral Transform and Solitons: Tools to Solve and Investigate Nonlinear Evolution Equations (North Holland, Amsterdam)

[34] Cantwell B J 2002 Introduction to Symmetry Analysis (CUP, Cambridge).

[35] Champagne B, W. Hereman W and Winternitz P 1991 The computer calculation of Lie point symmetries of large systems of differential equations Comput. Phys. Commun. 66 319-340.

[36] Champagne B and Winternitz P 1988 On the infinite dimensional symmetry group of the Davey-Stewartson equation J. Math. Phys. 29 1-8.

[37] Chen W Y C, Galbraith H W and Louck J D 2001 Angular momentum theory, umbral calculus, and combinatorics Comp. Math. Appl. 41 11991214 .

[38] Chiu S C and Ladik J F 1977 Generating exactly soluble nonlinear discrete evolution equations by a generalized Wronskian technique J. Math. Phys. 18 690-700.

[39] Cicogna G 2004 Weak symmetries and adapted variables for differential equations Int. J. Geom. Meth. Mod. Phys. 1 23-31.

[40] Cicogna G and Gaeta G 2001 Partial Lie-point symmetries of differential equations J. Phys. A: Math. Gen. 34 491-512.

[41] Clarkson P A and Kruskal M D 1989 New similarity reductions of the Boussinesq equation J. Math. Phys. 30 2201-2213.

[42] Clarkson P A and Nijhoff F W eds. 1999 Symmetries and integrability of difference equations LMS Lect. Note Series, vol. 255 (CUP, Cambridge)

[43] Conte R and Musette M 1996 A new method to test discrete Painlevé equations Phys. Lett. A223 439-448.

[44] Costin O and Kruskal M 2002 Movable singular points of solutions of difference equations, their relation to solvability, and the investigation of superstable fixed points Teoret. Mat. Fiz. 133 160-169.

[45] David D, Kamran N, Levi D and Winternitz P 1986 Subalgebras of loop algebras and symmetries of the Kadomtsev-Petviashvili equation Phys. Rev. Lett. 55 2111-2113.

[46] David D, Kamran N, Levi D and Winternitz P 1986 Symmetry reduction of the Kadomtsev-Petviashvili equation using a loop algebra J. Math. Phys. 27 1225-1237.

[47] DiBucchianico A and Loeb D 2000 Umbral Calculus Electr. J. Combin. DS3 http://www.combinatorics.org 
[48] Dimakis A, F Müller-Hoissen F and Striker T 1996 Umbral calculus, discretization, and quantum mechanics on a lattice J. Phys. A: Math. Gen. 29 6861-6876.

[49] Dodd R K 1978 Generalised Backlund transformation for some non-linear partial differential-difference equations J. Phys. A: Math. Gen. 11 81-92.

[50] Dorodnitsyn V A 1991Transformation groups in a space of difference variables J. Sov. Math. 55 1490-1517.

[51] Dorodnitsyn V A 1993 Finite difference analog of the Noether theorem Dokl. Ak. Nauk 328 678-682.

[52] Dorodnitsyn V A 1994 Finite difference models entirely inheriting continuous symmetries of differential equations Int. J. Mod. Phys. C 5 723-734.

[53] Dorodnitsyn V A 1993 Symmetry of Finite Difference Equations, Chapter 17 of CRC Handbook of Lie Group Analysis of Differential Equations. Vol. I: Symmetries, exact solutions and conservation laws ed. Ibragimov N H (CRC Press, Baton Rouge).

[54] Dorodnitsyn V A 2001 Group Properties of Difference Equations (Fizmatlit, Moscow) (in Russian)

[55] Dorodnitsyn V A and Kozlov: R 2003 A heat transfer with a source: the complete set of invariant difference schemes J. Nonlinear Math. Phys. 10 16-50.

[56] Dorodnitsyn V A, Kozlov R, and Winternitz P 2000 Lie group classification of second order difference equations J. Math. Phys. 41 480-509.

[57] Dorodnitsyn V A, Kozlov R, and Winternitz P 2004 Continuous symmetries of Lagrangians and exact solutions of discrete equations J. Math. Phys. 45 336-359.

[58] Dorodnitsyn V A and Winternitz P 2000 Lie point symmetry preserving discretizations for variable coefficient Korteweg-de Vries equations Nonlinear Dynamics 22 49-59.

[59] Faddeev L D and Takhtajan L A 1987 Hamiltonian methods in the theory of solitons (Springer-Verlag, Berlin) .

[60] Flaschka H 1974 The Toda lattice I. Existence of integrals Phys. Rev. B 9 1924-1925.

[61] Flaschka H 1974 On the Toda lattice II. Inverse Scattering Solutions Progr Theor Phys 51 703-716.

[62] Fermi E, Pasta J and Ulam S 1955 Los Alamos Rpt. LA1940 included in 1965 Fermi, Enrico Collected papers (Note e memorie). Vol. II: United States, 1939-1954. (The University of Chicago Press, Chicago, Ill.; Accademia Nazionale dei Lincei, Rome). 
[63] Floreanini R, Negro J, Nieto L M, and Vinet L 1996 Symmetries of the heat equation on a lattice Lett. Math. Phys. 36 351-355.

[64] Floreanini R and Vinet L 1995 Lie symmetries of finite difference equations J. Math. Phys. 36 7024-7042.

[65] Fokas A S 1980 A symmetry approach to exactly solvable evolution equations J. Math. Phys. 21 1318-1325.

[66] Fordy A P and Gibbons J 1980 Integrable nonlinear Klein-Gordon equations and Toda lattices, Commun. Math. Phys. 77 2-30.

[67] Fushchich V I, Serov N I and Chopik V I 1988 Conditional invariance and nonlinear heat equations. (Russian. English summary) Dokl. Akad. Nauk Ukrain. SSR Ser. A 86 17-21.

[68] Fushchich V I, Shtelen W M and Slavutsky S L 1991 Reduction and exact solutions of the NavierStokes equations W.I. J. Phys. A: Math. Gen. 24 971-984.

[69] Fushchich W I and Shtelen W M 1989 On approximate symmetry and approximate solutions of the non-linear wave equation with a small parameter J. Phys. A: Math. Gen. 22 L887-L890.

[70] Fuchssteiner B 1979 Application of hereditary symmetries to nonlinear evolution equations Nonlinear Anal. Theory Math. Appl. 3 849-862.

[71] Fuchssteiner B 1983 Mastersymmetries, high order time-dependent symmetries and conserved densities of nonlinear evolution equations Prog. Theor. Phys. 70 1508-1522.

[72] Fuchssteiner B and Fokas A S 1981/82 Symplectic structures, their Bäcklund transformations and hereditary symmetries Physica D 4 47-66.

[73] Fuchssteiner B 1981 The Lie algebra structure of nonlinear evolution equations admitting infinite-dimensional abelian symmetry groups Progr. Theoret. Phys. 65 (1981) 861-876.

[74] Gaeta G 1994 Nonlinear Symmetries and Nonlinear Equations (Kluwer, Dordrecht)

[75] Gaeta G 1994 Asymptotic symmetries and asymptotically symmetric solutions of partial differential equations J. Phys. A: Math. Gen. 27 437-451.

[76] Gaeta G, Levi D and Mancinelli R 2005 Asymptotic symmetries of difference equations, to be published.

[77] Gaeta G and Mancinelli R 2004 Asymptotic symmetries of diffusion and reaction-diffusion equations Preprint. 
[78] Gazeau J P and Winternitz P 1992 Symmetries of variable coefficient Korteweg-de Vries equation J. Math. Phys. 33 4087-4102.

[79] Gomez-Ullate D, Lafortune S and Winternitz P 1999 Symmetries of discrete dynamical systems involving two species J. Math. Phys. 40 27822804 .

[80] Gomez Ullate D, Tempesta P and Winternitz P eds. 2005 Proceedings of Workshop on Group Theory and Numerical Analysis, Montreal 26-31, May 2003 CRM Proc. and Lect. Notes, in press (AMS, Providence).

[81] Gonzalez-Lopez A, Kamran N and Olver P J 1992 Lie algebras of vector fields in the real plane Proc. London Math. Soc. 64 339-368.

[82] Gorskii A Z and Szmigielski 1998 On pairs of difference operators satisfying $[D, x]=I d, J$. Math. Phys. 39 568-585.

[83] Grammaticos B and Ramani R 2004 Discrete Painlevé Equations: a Review in Discrete Integrable Systems, Lecture Notes in Physics N.644, ed. by Grammaticos B, Kosmann-Schwarzbach Y and Tamizhmani T (Springer, Berlin) 245-321.

[84] Grammaticos G, Ramani A and Papageorgiou V 1991 Do integrable mappings have the Painlevé property Phys. Rev. Lett. 671825 - 1828.

[85] Grammaticos B,Tamizhmani T, Ramani A and Tamizhmani K M 2001 Growth and integrability in discrete systems J. Phys. A : Mat. Gen.34 $3811-3821$.

[86] Haggar F A, Byrnes G B and Quispel G R W 1996 K-integrals and k-Lie symmetries in discrete dynamical systems Physica A 233 379-394.

[87] Helgason S 1962 Differential Geometry and Symmetric Spaces (AP, New York)

[88] Hereman W 1996 Symbolic software for Lie symmetry analysis. In Lie Group Analysis of Differential Equations, vol. 3, ed. by N.H. Ibragimov (CRC Press, Boca Raton) 367-413

[89] Hernández Heredero R and Levi D 2003 The Discrete Nonlinear Schrödinger Equation and its Lie symmetry reductions J. Nonl. Math. Phys. 10 Suppl. 2 77-94.

[90] Hernandez Heredero R, Levi D, Rodriguez M A, and Winternitz P 2000 Lie algebra contractions and symmetries of the Toda hierarchy J. Phys. A: Math. Gen. 33 5025-5040.

[91] Hernandez Heredero R, Levi D, Rodriguez M A, and Winternitz P 2001 Relation between Bäcklund transformations and higher continuous symmetries of the Toda equation J. Phys. A: Math. Gen. 34 2459-2465. 
[92] Hernandez Heredero R, Levi D, Rodriguez M A and Winternitz P 2001 Symmetries of Differential Difference equations and Lie Algebra Contractions Bäcklund 83 Darboux Transformations:The Geometry of Soliton Theory eds. Coley A, Levi D, Milson R, Rogers C and Winternitz P (AMS, Providence).

[93] Hernandez Heredero R, Levi D, and Winternitz P 1999 Symmetries of the discrete Burgers equation J. Phys. A: Math. Gen. A32 2685-2695.

[94] Hernandez Heredero R, Levi D and Winternitz P 2000 Symmetry Preserving Discretization of the Burgers Equation Symmetries and integrability of difference equations eds. Levi D and Ragnisco O (AMS, Providence) 197-208.

[95] Hernandez Heredero R, Levi D, and Winternitz P 2001 Symmetries of the discrete nonlinear Schrödinger equation Theor. Math. Phys. 127 729-737.

[96] Hietarinta J 2001 Integrability tests for difference equations, Nonlinear Analysis 47 4641-4650.

[97] Hietarinta J and Viallet C 1998 Singularity Confinement and Chaos in Discrete Systems, Phys. Rev. Lett. 81 325-328.

[98] Hietarinta J and Viallet C 2000 Discrete Painlevé I and singularity confinement in projective space, Chaos, Solitons \& Fractals 11 29-32.

[99] Hirota R 1977 Nonlinear partial difference equations. I. A difference analogue of the Korteweg-de Vries equation, J. Phys. Soc. Japan 43 14241433.

[100] Hirota R 1977 Nonlinear partial difference equations. II. discrete-time Toda equation. J. Phys. Soc. Japan 43 2074-2078.

[101] Hirota R 1977 Nonlinear partial difference equations. III. discrete sineGordon equation J. Phys. Soc. Japan 43 2079-2086

[102] Hirota R 1978 Nonlinear partial difference equations. IV. Bäcklund transformations for the discrete-time Toda equation, J. Phys. Soc. Japan 45 $321-332$.

[103] Hirota R 1979 Nonlinear partial difference equations V. Nonlinear equations reducible to linear equations J. Phys. Soc. Japan 46 312-319.

[104] Hydon P E 2000 Symmetry Methods for Differential Equations (CUP, Cambridge).

[105] Inönü E and Wigner E P 1953 On the contraction of groups and their representations Proc. Nat. Ac. Sci. US 39 510-524.

[106] Ibragimov N H 1985 Transformation Groups Applied to Mathematical Physics (Reidel, Boston). 
[107] Ibragimov N H ed. 1993 CRC Handbook of Lie Group Analysis of Differential Equations. Vol. I: Symmetries, exact solutions and conservation laws (CRC Press, Baton Rouge).

[108] Ibragimov N H ed. 1994 CRC Handbook of Lie Group Analysis of Differential Equations. Vol. II: Applications in Engineering and Physicas Sciences (CRC Press, Baton Rouge).

[109] Ibragimov N H ed. 1995 CRC Handbook of Lie Group Analysis of Differential Equations. Vol. III (CRC Press, Baton Rouge).

[110] Izmest'ev A A, Pogosyan G S, Sissakian A N and Winternitz P 1999 Contractions of Lie algebras and separation of variables. The $n$-dimensional sphere J. Math. Phys. 40 1549-1573.

[111] Jacobson N 1962 Lie Algebras (Interscience, New York).

[112] Jiang Z-H 1998 Lie Symmetries and Their Local Determinancy for a Class of Differential-Difference Equations, Phys. Lett. A 240 137-143.

[113] Joshi N and Vassiliou P J 1995 The Existence of Lie Symmetries for First Order Analytic Discrete Dynamical Systems, J. Math. Anal. Appl. 195, 872-887.

[114] Kersten P H M and Krasil'shchik I S 2000 Symmetries and Recursion Operators for Classical and Supersymmetric Differential Equations (Kluwer Academic Publisher, Dordrecht).

[115] Kowalevski S 1889 Sur le Probléme de la Rotation d'un Corps Solid Autour d'un Point Fixé Acta Math. 12 177-232.

[116] Kowalevski S 1889 Sur une Propriété d'un Systéme d'Équations Différentielles qui Definit la Rotation d'un Corps Solide Autour d'un Point Fixé Acta Math. 14 81-93.

[117] Kruskal M D, Joshi N and Halburd R 2004 Analytic and asymptotic methods for nonlinear singularity analysis: a review and extensions of tests for the Painleve property, in Integrability of Nonlinear Systems eds. Kosmann-Schwarzbach Y, Grammaticos B and Tamizhmani K M (Springer-Verlag, Berlin) 171-205.

[118] Lafortune S, Martina L and Winternitz P 2000 Point symmetries of generalized Toda field theories J. Phys. A: Math. Gen. 33 2419-2435.

[119] Lafortune S, Tremblay S and Winternitz P 2001 Symmetry classification of diatomic molecular chains J. Math. Phys. 42 5341-5357.

[120] Lamb J S W and Quispel G R W 1995 Cyclic reversing k-symmetry groups Nonlinearity 8 1005-1026. 
[121] Lax P D 1968 Integrals of nonlinear equations of evolution and solitary waves Comm. Pure Appl. Math. 21 467-490.

[122] Levi D 1981 Nonlinear differential difference equations as Bäcklund transformations J. Phys. A: Math.Gen. 14 1083-1098.

[123] Levi D 1992 Inhomogeneous Toda lattices and their symmetries in Group theory in physics, eds. Frank A, Seligman T H and Wolf K B ( AIP, New York ) 97-108.

[124] Levi D 1998 Symmetries and generalized symmetries for discrete dynamical systems Symmetries and Integrability of Difference Equations eds. Clarkson P A and Nijhoff F W (CUP, Cambridge) 261-274.

[125] Levi D 2003 Lie symmetries for lattice equations Group 24: Physical and Mathematical Aspects of Symmetries eds. Gazeau J-P, Kerner R, Antoine J-P, Metens S and Thibon J-Y ( IOP Publishing, Bristol) 103-111.

[126] Levi D and Benguria R 1980 Bäcklund transformations as nonlinear differential difference equations Proc. Natl. Acad. Sci (USA) 77 307-311.

[127] Levi D and Martina L 2001 Integrable hierarchies of nonlinear differencedifference equations and symmetries J. Phys. A: Math. Gen. 34 1035710368.

[128] Levi D, Negro J and del Olmo M A 2001 Discrete derivatives and symmetries of difference equations, J. Phys. A: Math. Gen. 34 2023-2030.

[129] Levi D, Negro J and del Olmo M A 2001 Lie symmetries of difference equations, Czech. J. Phys. 51 341-348.

[130] Levi D, Negro J and del Olmo M A 2004 Discrete q-derivatives and symmetries of q-difference equations, J. Phys. A: Math. Gen. 37 3459-3473.

[131] Levi D, Pilloni L and Santini P M 1981 Integrable three-dimensional lattices J. Phys. A: Math. Gen. 14 1567-75.

[132] Levi D and Ragnisco O 1979 Non-linear differential-difference equations with $N$-dependent coefficients II J. Phys. A: Math. Gen. 12 L163-L167.

[133] Levi D and Ragnisco O 1991 The inhomogeneous Toda lattice: its hierarchy and Darboux-Bäcklund transformations. J. Phys. A: Math.Gen. 24 1729-1739.

[134] D. Levi and O. Ragnisco eds. 2000 SIDE III-Symmetries and integrability of difference equations CRM Proc. and Lect. Notes, vol. 25 (AMS, Providence).

[135] Levi D, Ragnisco O and Rodríguez M A 1992 On non-isospectral flows, Painlevé equations and symmetries of differential and difference equations Teor. Mat. Fizika 93 473-480 
[136] Levi D and Rodriguez M A 1992 Symmetry group of partial differential equations and of difference equations: the Toda lattice vs the Kortewegde Vries equation J. Phys. A: Math. Gen. 25 L975-L979.

[137] Levi D and Rodríguez M A 1999 Lie symmetries for integrable evolution equations on the lattice J. Phys. A: Math. Gen. 32 8303-8316.

[138] Levi D and Rodríguez M A, 2004 Lie discrete symmetries of lattice equations J. Phys. A: Math. Gen. 37 1711-1725

[139] Levi D, Tempesta P and Winternitz P 2004 Umbral calculus, difference equations and the discrete Schrödinger equation J. Math. Phys. 45 40774105 .

[140] Levi D, Tempesta P and Winternitz P 2004 Lorentz and Galilei Invariance on Lattices Phys. Rev. D 69105011 pp. 1-6.

[141] Levi D, Tremblay S and Winternitz P 2001 Lie point symmetries of difference equations and lattices J. Phys. A: Math. Gen. 33 8507-8524.

[142] Levi D, Tremblay S and Winternitz P 2001 Continuous symmetries of equations on lattices Czech. J. Phys. 51 349-356.

[143] Levi D, Tremblay S and Winternitz P 2001 Lie Symmetries of multidimensional difference equations J. Phys. A: Math. Gen. 34 9507-9524.

[144] Levi D, Vinet L and Winternitz P eds. 1996 Symmetries and integrability of difference equations CRM Proc. and Lect. Notes, vol. 9 (AMS, Providence).

[145] Levi D, Vinet L and Winternitz P 1997 Lie group formalism for difference equations J. Phys. A: Math. Gen. 30 663-649.

[146] Levi D and Winternitz P 1988 The cylindrical Kadomtsev-Petviashvili equation: its Kac-Moody-Virasoro algebra and relation to KP equation Phys. Lett. A 129 165-167.

[147] Levi D and Winternitz P 1989 Nonclassical symmetry reduction: Example of the Boussinesq equation J. Phys. A: Math. Gen. 22 2915-2924.

[148] Levi D and Winternitz P 1991 Continuous symmetries of discrete equations Phys. Lett. A 152 335-338.

[149] Levi D and Winternitz P 1993 Symmetries of differential equations on a lattice. An example: the Toda lattice Modern Group Analysis: Advanced Analytical and Computational Methods in Mathematical Physics eds Ibragimov N H, Torrisi M and Valenti A (Kluwer Academic Publisher, Dordrecht) 265-272. 
[150] Levi D and Winternitz P 1993 Dimensional reduction for equations involving discrete and continuous variables Applications of Analytic and geometric Methods to Nonlinear Differential Equations ed. Clarkson P A (Kluwer Academic Publishers, Dordrecht) 405-413.

[151] Levi D and Winternitz P 1993 Symmetries and conditional symmetries of differential-difference equations J. Math. Phys. 34 3713-3730.

[152] Levi D and Winternitz P 1995 Continuous symmetries of differential difference equations Modern Group Theoretical Methods in Physics eds. Bertrand J, Flato M, Gazeau J-P, Irac-Astaud M and Sternheimer D (Kluwer Academic Publisher, Dordrecht) 271-282.

[153] Levi D and Winternitz P 1996 Symmetry analysis of discrete physical systems Nonlinear Physics, Theory and Experiment eds. Alfinito E, Boiti M, Martina L and Pempinelli F (World Scientific, Singapore) 199-216.

[154] Levi D and Winternitz P 1996 Lie point symmetries of differential difference equations Symmetries and Integrability of Difference Equations eds. Levi D, Vinet L and Winternitz P (AMS, Providence) 199-208.

[155] Levi D and Winternitz P 1996 Symmetries of discrete physical systems Proceedings of the IV Wigner Symposium eds. Atakishiyev N M, Seligman T H and Wolf K B (World Scientific, Singapore) 382-391.

[156] Levi D and Winternitz P 2002 Symmetries of discrete dynamical systems, J. Math. Phys. 37 5551-5576.

[157] Levi D and Winternitz P 2002 Lie point symmetries and commuting flows for equations on lattices, J. Phys. A: Math. Gen. 35 2249-2262.

[158] Levi D and Yamilov R 1997 Conditions for the Existence of Higher Symmetries of Evolutionary Equations on a Lattice J. Math. Phys. 38 6648-6674

[159] Levi D and Yamilov R 1999 Dilation symmetries and equations on the lattice. J. Phys. A: Math. Gen. 32 8317-8323.

[160] Levi D and Yamilov R 2000 Non-point integrable symmetries for equations on the lattice. J. Phys. A: Math. Gen. 33 4809-4823.

[161] Levi D and Yamilov R 2001 On the integrability of a new discrete nonlinear Schrödinger equation. J. Math. Phys. 34 L553-L562.

[162] Li Y-s and Zhu G-c 1986 New set of symmetries of the integrable equations, Lie algebra and non-isospectral evolution equations. II. AKNS system $J$. Phys. A: Math. Gen. 19 3713-3725.

[163] Lie S 1924 Klassifikation und Integration von gewohnlichen Differentialgleichunden zwischen x, y die eine Gruppe von Transformationen gestatten. $I-I V$. Gessamelte Abhandlungen (Teubner, Leipzig), Vol. 5. 
[164] Lie S 1891 Vorlesungen uber Differentialgleichungen mit bekannten infinitesimalen Transformationen, bearbeitet und herausgegeben von Dr. G. Scheffers (Teubner, Leipzig), reprinted 1967 as Differentialgleichungen (Chelsea, New York).

[165] Lie S 1893 Vorlesungen uber continuirliche Gruppen mit geometrischen und anderen Anwendungen, bearbeited und herausgegeben von Dr. G. Scheffers (Teubner, Leipzig).

[166] Ma W-X and Fuchssteiner B 1999 Algebraic structure of discrete zero curvature equations and master symmetries of discrete evolution equations J. Math. Phys. 40 2400-2418.

[167] Maeda S 1980 Canonical structure and symmetries for discrete systems Math. Japan 25 405-420.

[168] Maeda S 1981 Extension of discrete Noether theorem. Math. Japon. 26 85-90.

[169] Maeda S 1982 Lagrangian formulation of discrete systems and concept of difference space. Math. Japon. 27 345-356.

[170] Maeda s 1987 The similarity method for difference equations IMA J. Appl. Math. 38 129-134.

[171] Maeda S 1991 Application of numerical schemes admitting symmetries. Electron. Comm. Japan Part III Fund. Electron. Sci. 73 107-113.

[172] Maillet J-M and Nijhoff F W 1989 Integrability for multidimensional lattice models Phys. Lett. B 224 389-396.

[173] Mahomed F M and Leach P G L 1989 Lie algebras associated with scalar second-order ordinary differential equations J. Math. Phys. 30, 2770-2777.

[174] Mahomed F M and Leach P G L 1989 The Lie algebra sl(3,R) and linearization Quaest. Math. 12 121-139.

[175] Manakov S V 1975 Complete integrability and stochastization of discrete dynamical systems Soviet Phys. JETP 40 269-274.

[176] Martina L, Lafortune S and Winternitz P 2000 Point symmetries of generalized Toda field theories: II. Symmetry reduction J. Phys. A: Math. Gen. 33 6431-6446.

[177] Martina L and Winternitz P 1989 Analysis and applications of the symmetry group of the multidimensional three-wave resonant interaction problem, Ann. Phys. $196231-277$.

[178] Mikhailov A V 1979 Integrability of a two-dimensional generalization of the Toda chain, Sov. Phys. JETP Lett. 30 414-418. 
[179] Milne-Thomson L M 1933 The calculus of finite differences, (Macmillan, London).

[180] Moody R V and Patera J 1991 Discrete and continuous graded contractions of representations of Lie algebras J. Phys. A: Math. Gen. 24 22272258 .

[181] Naimark M A and Stern A I 1982 Theory of Group Representations (Springer-Verlag, New York).

[182] Newell A C 1985 Solitons in Mathematics and Physics (SIAM, Philadelphia).

[183] Niederer U 1973 The maximal kinematical invariance group of the Schrödinger equation Helv. Phys. Acta 45 802-810.

[184] Nijhoff F W 1999 Discrete Painlevé equations and symmetry reduction on the lattice. in Discrete integrable geometry and physics eds. Bobenko A and Seiler R (OUP, New York) 209-234 .

[185] Nijhoff F W and Capel H W 1990 The direct linearisation approach to hierarchies of integrable PDEs in $2+1$ dimensions. I. Lattice equations and the differential-difference hierarchies Inverse Problems 6 567-590.

[186] Nijhoff F and Capel H W 1995 The discrete Korteweg de Vries equation. In $K d V$ '95 eds. Hazewinkel M, Capel $\mathrm{H} \mathrm{W}$ and de Jager E M (Kluwer, Dordrecht).

[187] Nijhoff F W, Papageorgiou V G and Capel H W 1992 Integrable timediscrete systems: lattices and mappings. in Quantum groups, Proc. Workshops, Euler Int. Math. Inst. Leningrad USSR 1990 (Lecture Notes in Mathematics Vol. 1510) 312-25.

[188] Nijhoff F W, Papageorgiou V G, Capel H W and Quispel G R W 1992 The lattice Gelfand-Dikii hierarchy Inverse Problems 8 597-621.

[189] Nijhoff F W, Suris Yu, Viallet C eds. 2003 SIDE V-Symmetries and integrability of difference equations J. Nonl. Math. Phys. 10 Supplement 2, $1-245$.

[190] Nimmo J J C and Schief W K 1997 Superposition principles associated with the Moutard transformation: an integrable discretization of a $(2+1)$ dimensional sine-Gordon system Proc. R. Soc. Lond. A 453 255-279.

[191] Noether E 1918 Invariante Variationsprobleme Nachr. Ges. Wiss. Goettingen, Math.-Phys. Kl. 2 (1918) 235-257 [For an English translation see 1971 Transp. Theory Stat. Phys. 1 186].

[192] Novikov S, Manakov S V, Pitaevskii L P and Zakharov V E 1984 Theory of solitons. The inverse scattering method (Consultants Bureau [Plenum], New York). 
[193] Ohta Y, Tamizhmani K M, Grammaticos B and Ramani A 1999 Singularity confinement and algebraic entropy: the case of the discrete Painlevé equations Phys. Lett. A $\mathbf{2 6 2} 152-157$.

[194] Olver P J 1993 Applications of Lie Groups to Differential Equations (Springer-Verlag, New York)

[195] Olver P J and Rosenau Ph 1987 Group invariant solutions of differential equations SIAM J. Appl. Math. 47 263-278.

[196] Orfanidis S J 1978 Discrete sineGordon equations Phys. Rev. D $183822-$ 3827.

[197] Orlov A Yu and Winternitz P 1997 Algebra of pseudodifferential operators and symmetries of equations in the KadomtsevPetviashvili hierarchy, $J$. Math. Phys. 38 4644-4674.

[198] Ovsiannikov L V 1982 Group Analysis of Differential Equations (AP, New York).

[199] Penskoi A V and Winternitz P. 2004 Discrete Riccati equations with superposition formulas J. Math. Anal. Appl. 294 533-547.

[200] Quispel G R W, Capel H W and Sahadevan R 1992 Continuous symmetries of difference equations; the Kac-van Moerbeke equation and Painlevé reduction Phys. Lett. A 170 379-383.

[201] Quispel G R W and Sahadevan R 1993 Lie symmetries and integration of difference equations Phys. Lett. A 184 64-70.

[202] Ramani A and Grammaticos B 2002 What is the discrete analogue of the Painlevé property?ANZIAM J. 44 21-32.

[203] Ramani A, Grammaticos B and Tamizhmani K M 1993 Painlevé analysis and singularity confinement: the ultimate conjecture. J. Phys. A : Math. Gen. 26 L53-L58.

[204] Ramani A, Grammaticos B, Tamizhmani T and Tamizhmani K M 2003 The road to the discrete analogue of the Painlevé property: Nevanlinna meets singularity confinement. Advances in difference equations, IV Comput. Math. Appl. 45 1001-1012.

[205] Rand D W, Winternitz P, and Zassenhaus H 1988 On the identification of a Lie algebra given by its structure constants. I. Direct decompositions, Levi decomposition and nilradicals Lin. Algebra Appl. 109, 197-246.

[206] Remoissenet M 1999 Waves called solitons. Concepts and experiments (Springer-Verlag, Berlin).

[207] Rodriguez M A and Winternitz P 2004 Lie symmetries and exact solutions of first order difference schemes J. Phys. A: Math. Gen. 37, 6129-6142. 
[208] Rogers C and Ames W F 1989 Nonlinear Boundary Value Problems in Science and Engineering (AP, Boston).

[209] Rogers C, Schief W and Winternitz P 1997 Lie theoretical generalization and discretization of the Pinney equation J. Math. Anal. Appl. 216 246264.

[210] Roman S 1984 The Umbral Calculus (AP, San Diego)

[211] Rota G C 1975 Finite Operator Calculus (AP, San Diego)

[212] Rota G C 2003 Gian-Carlo Rota on analysis and probability. Selected papers and commentaries. Edited by Dhombres J, Kung J P S and Starr N (Birkhäuser, Boston).

[213] Sahadevan R 2001 Nonlinear Differential-Difference and Difference Equations: Integrability and Exact Solvability Comp. Math. Appl. 42 627-637.

[214] Sahadevan R, Byrnes G B and Quispel G R W 1994 Linearisation of difference equations using factorisable Lie symmetries Proc. Workshop on Symmetries and Integrability of Difference Equation 1994. Esterel Montreal Canada eds. Levi D, Vinet L and Wintemitz P (AMS, Providence).

[215] Sahadevan R and Khousalya S 2000 Similarity reduction of a $(2+1)$ Volterra J. Phys. A: Math. Gen. 33 L171-L176.

[216] Sahadevan R and Quispel G R W 1997 Lie symmetries and linearization of the QRT mapping Physica A 234 775-784.

[217] Saito S, Saitoh N, Yamamoto J and Yoshida K 2001 A characterization of discrete time soliton equations J. Phys. Soc. Japn. 70 3517-3523.

[218] Satsuma J and Tokihiro T eds. 2001 SIDE IV-Symmetries and integrability of difference equations J. Phys. A: Math. Gen. 34 10337-10774.

[219] Schief W K 1997 A Discrete Pinney Equation Appl. Math. Lett. 10 13-15.

[220] Schief W K and Rogers C 2000 Bäcklund transformations and the integrable discretization of characteristic equations Chaos Sol. Fract. 11 $107-113$.

[221] Schwarz F 1982 A REDUCE package for determining Lie symmetries of ordinary and partial differential equations Comput. Phys. Commun. 27, $179-186$.

[222] Shabat A B 2003 Universal Models of Soliton Hierarchies Theor. Math. Phys. 136 1066-1076.

[223] Shabat A B and Yamilov R I 1991 Symmetries of nonlinear chains Leningrad Math. J. 2 377-400. 
[224] Steeb W-H 1996 Continuous Symmetries, Lie Algebras, Differential Equations and Computer Algebra (World Scientific, Singapore).

[225] Stephani H 1990 Differential Equations: Their Solution Using Symmetries ed. MacCallum M (CUP, Cambridge).

[226] Stormark O 2000 Lie's Structural Approach to PDE Systems (CUP, Cambridge).

[227] Suris, Yu B 2003 The problem of integrable discretization: Hamiltonian approach. (Birkhäuser, Basel).

[228] Tamizhmani K M, Ramani A, Grammaticos B and Ohta Y 1999 Integrability criteria for differential-difference systems: a comparison of singularity confinement and low-growth requirements J. Phys. A : Math. Gen. 32 6679-6685.

[229] Toda T 1967 Vibration of a Chain with Nonlinear Interaction J. Phys. Soc. Jpn. 22 431-436.

[230] Toda M 1981 Theory of nonlinear lattices (Springer Verlag, Berlin) .

[231] Toda M 1989 Nonlinear waves and solitons (Kluwer Academic Publishers, Dordrecht).

[232] Tremblay S, Grammaticos B and Ramani A 2001 Integrable lattice equations and their growth properties Phys. Lett. A 278 319-324.

[233] Turbiner A and Winternitz P 1999 Solutions of nonlinear ordinary differential and difference equations with superposition formulas Lett. Math. Phys. 50 189-201.

[234] Tzitzeica G 1907 Sur une nouvelle classes de surfaces, C. R. Acad. Sci. Paris 144 1257-1259.

[235] Tzitzeica G 1910 Sur une nouvelle classe de surfaces, C. R. Acad. Sci. Paris 150 955-956.

[236] Winternitz P 1993 Lie Groups and solutions of nonlinear partial differential equations. In: Integrable Systems, Quantum Groups and Quantum Fields Theories, eds. A. Ibort and M.A. Rodriguez (Kluwer, Dordrecht) 429-495.

[237] Winternitz P (1999) Lie groups and difference equations Group 22, Proceedings of the 22 International Colloquium on Group Theoretical Methods in Physics eds. Corney S P, Delbourgo R, and Jarvis P D (International Press, Boston) 113-124.

[238] Winternitz P 2004 Symmetries of discrete systems, in Discrete Integrable Systems, Lecture Notes in Physics N. 644, eds. B Grammaticos, KosmannSchwarzbach Y and Tamizhmani T (Springer, Berlin) 185-243. 
[239] Yamilov, R. I. 2004 Relativistic Toda chains and Schlesinger transformations. (in Russian) Teoret. Mat. Fiz. 139, 209-224.

[240] Yamilov R and Levi D 2004 Integrability Conditions for $n$ and $t$ Dependent Dynamical Lattice Equations, J. Nonl. Math. Phys. 11 75-101.

[241] Zakharov V E (Editor) 1991 What is Integrability? (Springer, New York) 